\title{
Node-Accessible Zirconium MOFs
}

Zhiyong Lu, ${ }^{*}, \uparrow, \S, \$$ Jian Liu, ${ }^{\S, \downarrow}$ Xuan Zhang, ${ }^{\S}$ Yijun Liao, ${ }^{\S}$ Rui Wang, ${ }^{\S}$ Kun Zhang, ${ }^{\S, P}$ Jiafei Lyu, ${ }^{\S, \phi}$ Omar K. Farha, ${ }^{\S,+}$ Joseph T. Hupp*,§

$\uparrow$ College of M echanics and M aterials, H ohai U niversity, N anjing 210098, C hina

$\S$ Department of Chemistry, N orthwestern U niversity, 2145 Sheridan Road, Evanston, Illinois 60208, USA

"School of Chemical Engineering, N anjing U niversity of Science\& T echnology, N anjing 210094, C hina

${ }^{\phi}$ Department of Pharmaceutical Engineering and Key Laboratory of Systems Bioengineering, Tianjin University, Tianjin, China

$\downarrow$ Department of Chemical and Biological Engineering, N orthwestern U niversity, Evanston, Illinois 60208, USA

\section{Table of contents}

1. Materials and General Methods

2. Characterization of NU-1000

3. Access to NU-1000-FF-Cl by Non-formate-generating Solvents

4. Access to NU-1000-FF-Cl at Room Temperature

5. Single-crystal Data

6. NU-1000-FF-Cl Converting Back to NU-1000-F (denoted as R1-NU-1000-F)

7. Access to NU-1000-FF by Base-washing

8. NU-1000-FF Converting Back to NU-1000-F (denoted as R2-NU-1000-F)

9. Hydrolysis experiments and ${ }^{31} \mathrm{P}$ NMR spectra

10. Catalyst Recyclability 


\section{Materials and General Methods}

Materials. All reagents were obtained from commercial vendors and, unless otherwise noted, were used without further purification. $\mathrm{H}_{4}$ TBAPy was synthesized following the published procedures.

\section{Physical Methods and Instrumentation.}

$\mathrm{N}_{2}$ isotherms were collected on a Micromeretics Tristar II 3020 instrument at 77 K. Pore-size distributions were calculated from these isotherms using a carbon split-pore model with a $\mathrm{N}_{2}$ kernal.

${ }^{1} \mathrm{H}$ NMR Spectroscopy. Samples were prepared by weighing $2 \mathrm{mg}$ of MOF into a $1.5 \mathrm{~mL}$ vial. About 3 to 4 drops of $0.1 \mathrm{M} \mathrm{NaOD}$ in $\mathrm{D}_{2} \mathrm{O}$ digestion medium was then added to the vials. The vials were capped and inverted 2 or 3 times before sonicating for $30 \mathrm{~min}$. This procedure dissolves only the organic portion of the MOF (linker and modulator); the inorganic component precipitates as zirconium oxide or hydroxide. To the mixture, 17 drops of $\mathrm{D}_{2} \mathrm{O}$ were added. Then the mixture was centrifuged and the clear supernatant solution was transferred to an NMR tube. ${ }^{1} \mathrm{H}$ NMR spectra were recorded with a Bruker Avance DPX-500 NMR spectrometer (500 MHz; 64 scans).

Diffuse reflectance infrared Fourier transform (DRIFT) spectra were recorded on a Nicolet 7600 FTIR spectrometer equipped with an MCT/A detector. Samples diluted in $\mathrm{KBr}$ were measured with a $\mathrm{KBr}$ background and kept at each temperature under Ar purge (samples prepared in atmosphere). The spectra were collected at $1 \mathrm{~cm}-1$ resolution and 32 scans were averaged over the spectral window of 675-4000 cm-1. All samples were activated samples, evaluated after BET surface area characterization.

Powder X-ray diffraction (PXRD) patterns were measured at room temperature on a STOESTADIMP powder diffractometer equipped with an asymmetric curved Germanium monochromator (CuKa1 radiation, $\lambda=1.54056 \AA$ ) and one-dimensional silicon strip detector (MYTHEN2 $1 \mathrm{~K}$ from DECTRIS). The line focused $\mathrm{Cu}$ X-ray tube was operated at $40 \mathrm{kV}$ and $40 \mathrm{~mA}$. The activated powder was sandwiched between two Kapton foils and measured in transmission geometry in a rotating holder. Intensity data from 2 to 30 degrees two theta were collected over a period of $6 \mathrm{~min}$. The instrument was calibrated against a NIST Silicon standard (640d) prior to the measurement.

Scanning electron micrographs (SEM) images were taken using a Hitachi SU8030/S-4800 at the EPIC facility (NUANCE Center-Northwestern University). EDS Map scans were obtained on the same instrument. 


\section{Characterization of NU-1000}

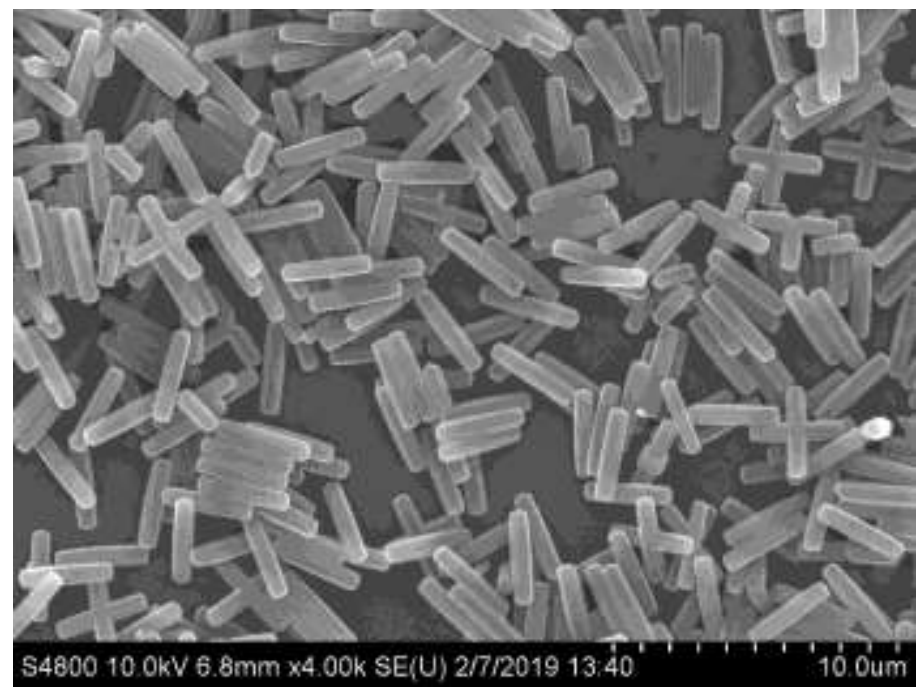

Figure S1. Scanning Electron Microscopy (SEM) image of NU-1000-F.

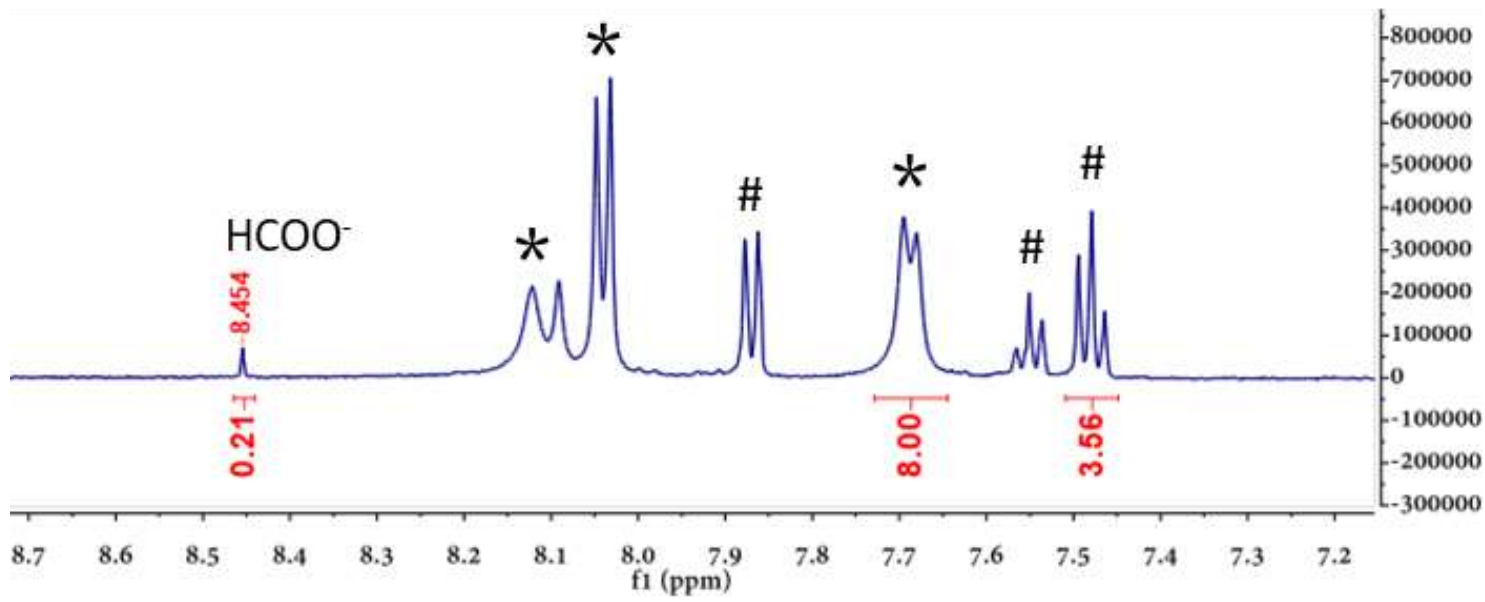

Figure S2. ${ }^{1} \mathrm{H}$ NMR spectrum of as-syn-NU-1000 digested in $0.1 \mathrm{M} \mathrm{NaOD} / \mathrm{D}_{2} \mathrm{O}$ and diluted in $\mathrm{D}_{2} \mathrm{O}$. Corresponding peaks of TBAPy ligand (*) and benzoate (\#). There are about 3.6 benzoate and 0.4 formate (at $8.454 \mathrm{ppm}$ ) ions per $\mathrm{Zr}_{6}$ node. 


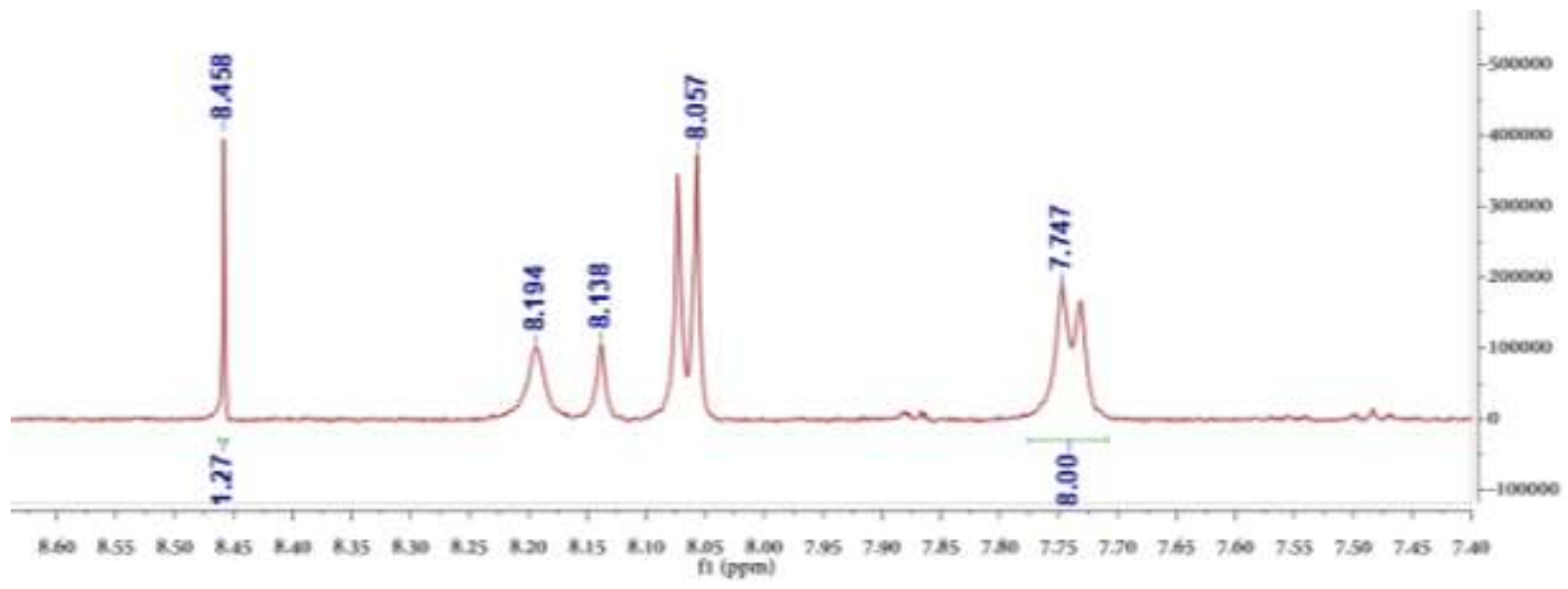

Figure S3. ${ }^{1} \mathrm{H}$ NMR spectrum of NU-1000-F digested in $0.1 \mathrm{M} \mathrm{NaOD} / \mathrm{D}_{2} \mathrm{O}$ and diluted in $\mathrm{D}_{2} \mathrm{O}$. There is no or negligible amount of benzoate ligand but 2.54 formate (at $8.458 \mathrm{ppm}$ ) per $\mathrm{Zr}_{6}$ node.

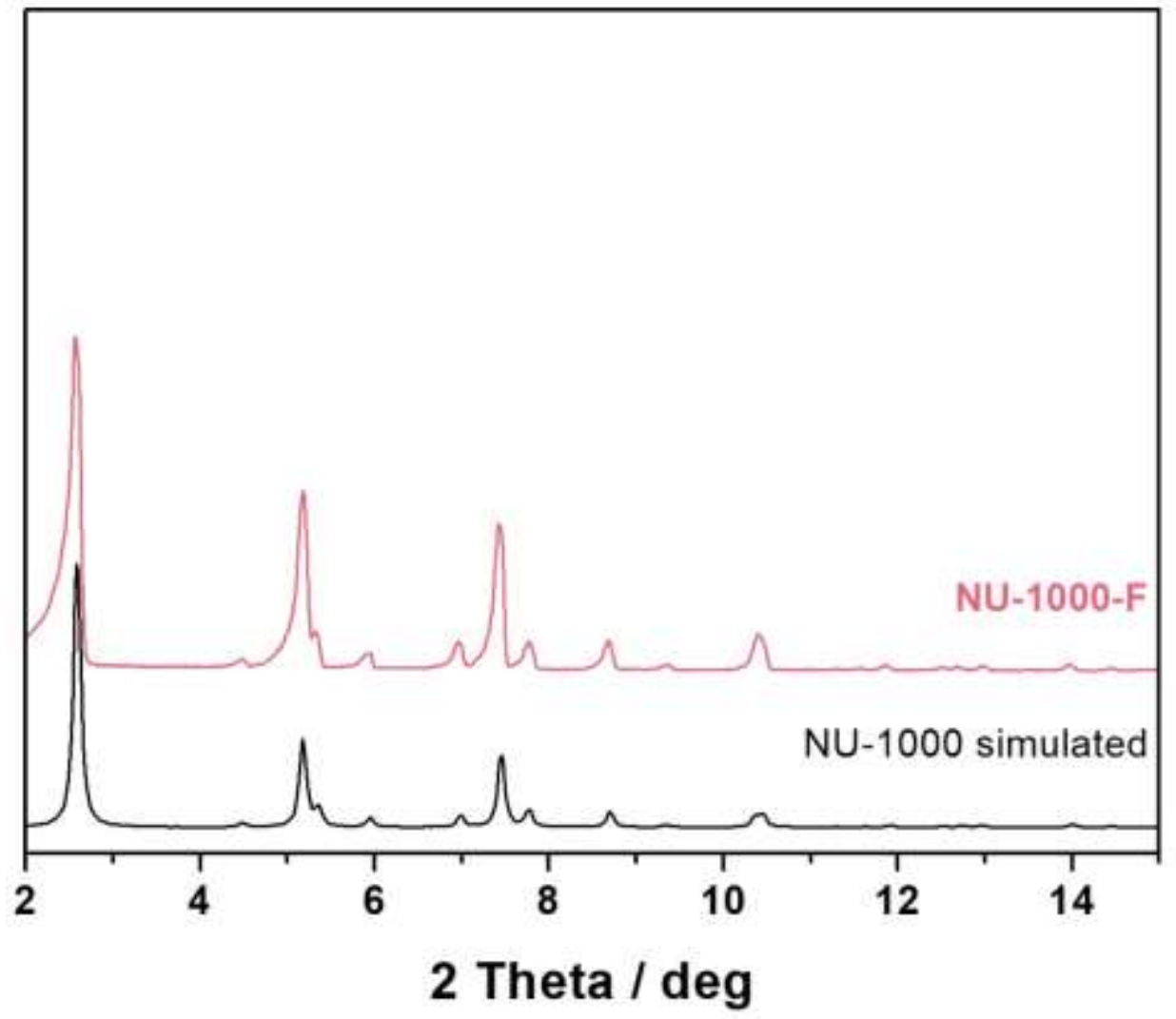

Figure S4. PXRD patterns of NU-1000-F (pink) and simulated (navy) 


\section{Access to NU-1000-FF-Cl by Non-formate-generating Solvents}
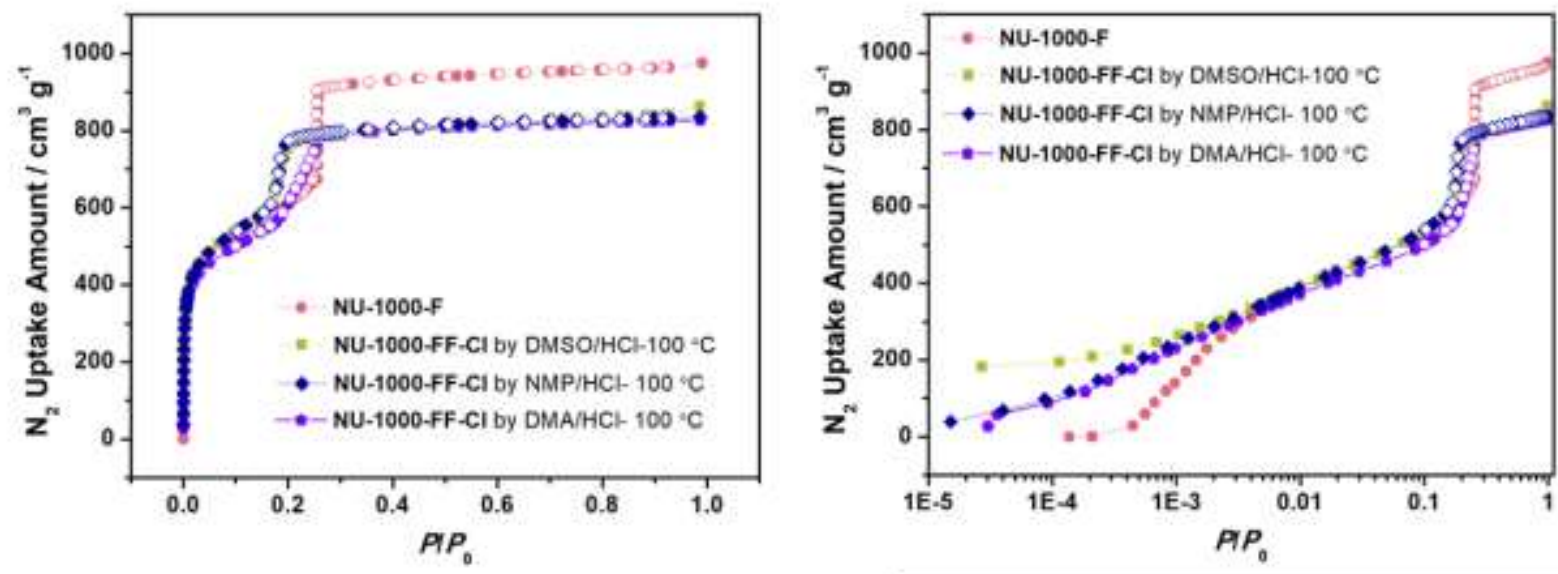

Figure S5. $\mathrm{N}_{2}$ adsorption isotherms at $77 \mathrm{~K}$ for NU-1000-F (green circle), and NU-1000-FF-Cl samples treated by different kinds of non-formate-generating solvent such as DMSO (violet square), NMP (navy diamond), and DMA (red pentagon).

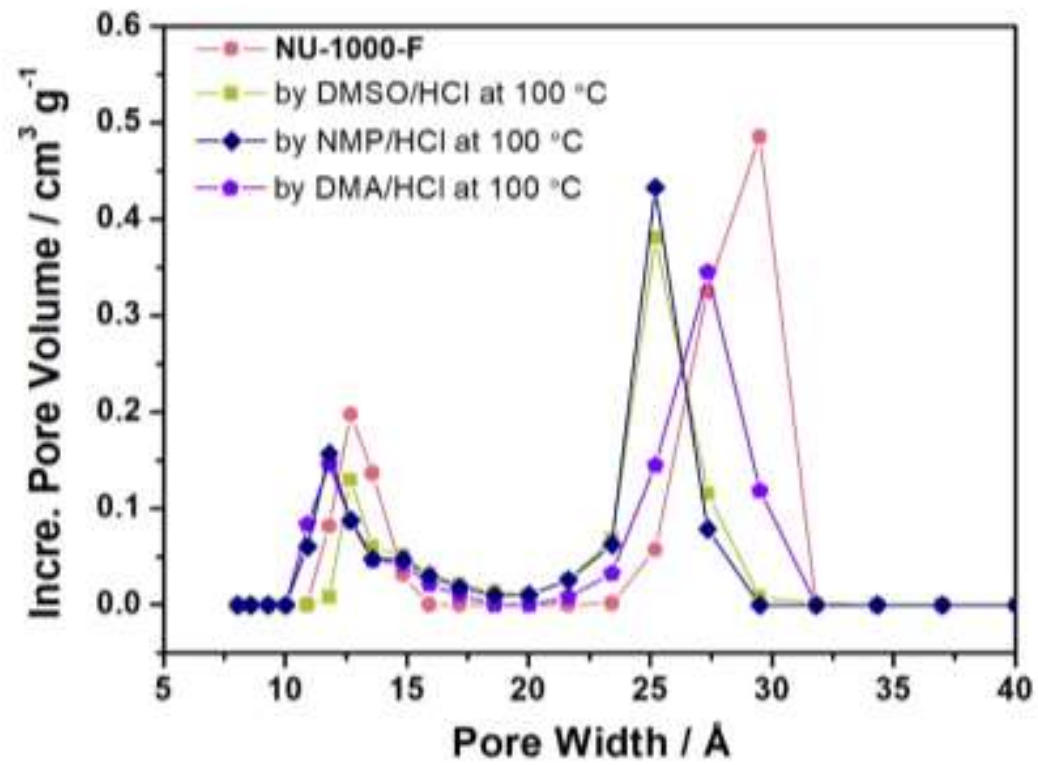

Figure S6. Pore size distributions of NU-1000-F (green circle), and NU-1000-FF-CI samples treated by different kinds of non-formate-generating solvent such as DMSO (violet square), NMP (navy diamond), and DMA (red pentagon). 


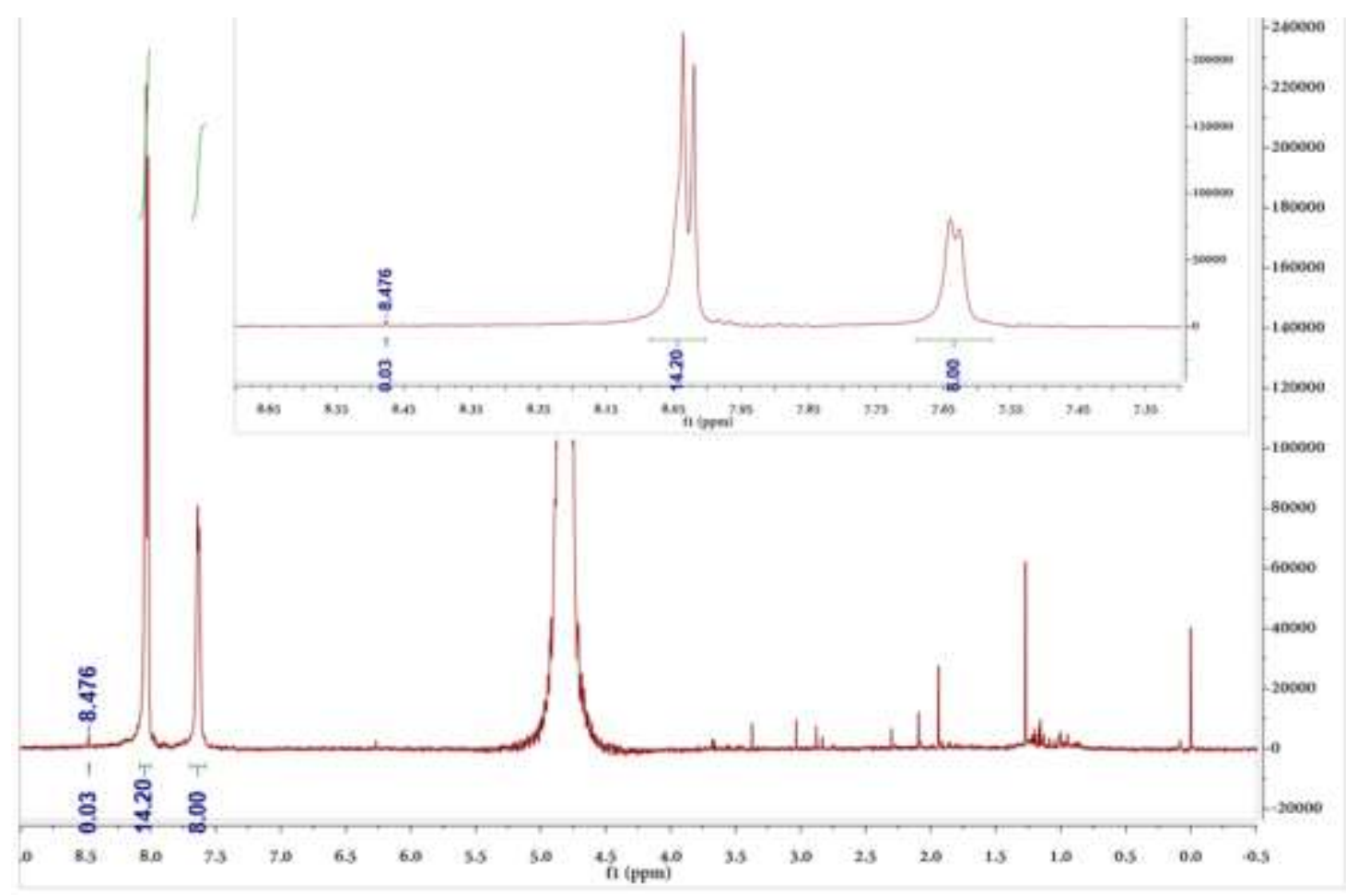

Figure S7. ${ }^{1} \mathrm{H}$ NMR spectrum of NU-1000-FF-Cl treated by $\mathrm{DMSO} / \mathrm{HCl}$ at $100{ }^{\circ} \mathrm{C}$. The sample was digested in $0.1 \mathrm{M} \mathrm{NaOD} / \mathrm{D}_{2} \mathrm{O}$ and diluted in $\mathrm{D}_{2} \mathrm{O}$. There is no detectable benzoate and only negligible formate (at $8.476 \mathrm{ppm})$.

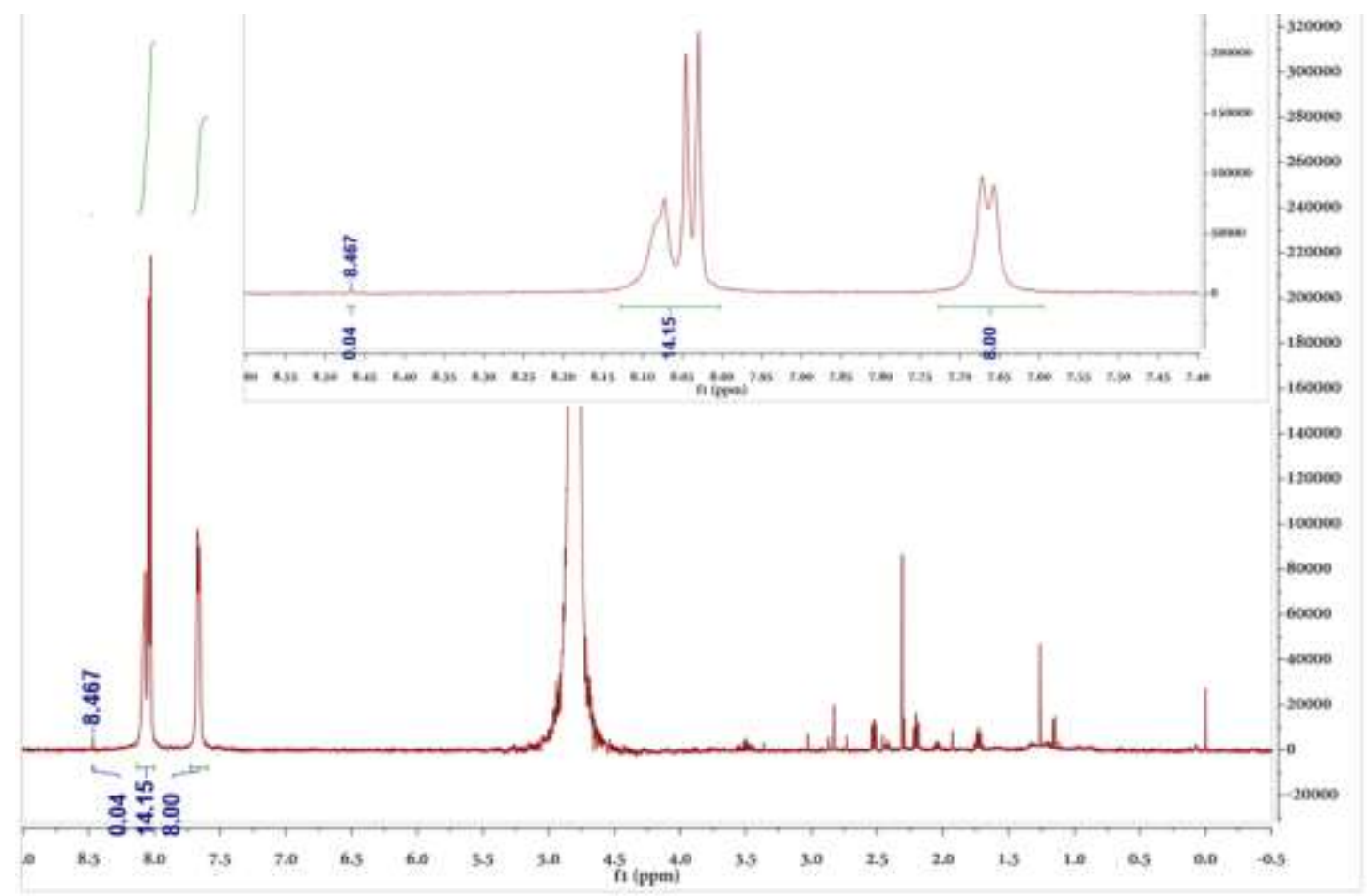

Figure S8. ${ }^{1} \mathrm{H}$ NMR spectrum of NU-1000-FF-Cl treated by $\mathrm{NMP} / \mathrm{HCl}$ at $100{ }^{\circ} \mathrm{C}$. The sample was digested in $0.1 \mathrm{M} \mathrm{NaOD} / \mathrm{D}_{2} \mathrm{O}$ and diluted in $\mathrm{D}_{2} \mathrm{O}$. There is no benzoate ligand and negligible formate (at $8.467 \mathrm{ppm})$. 


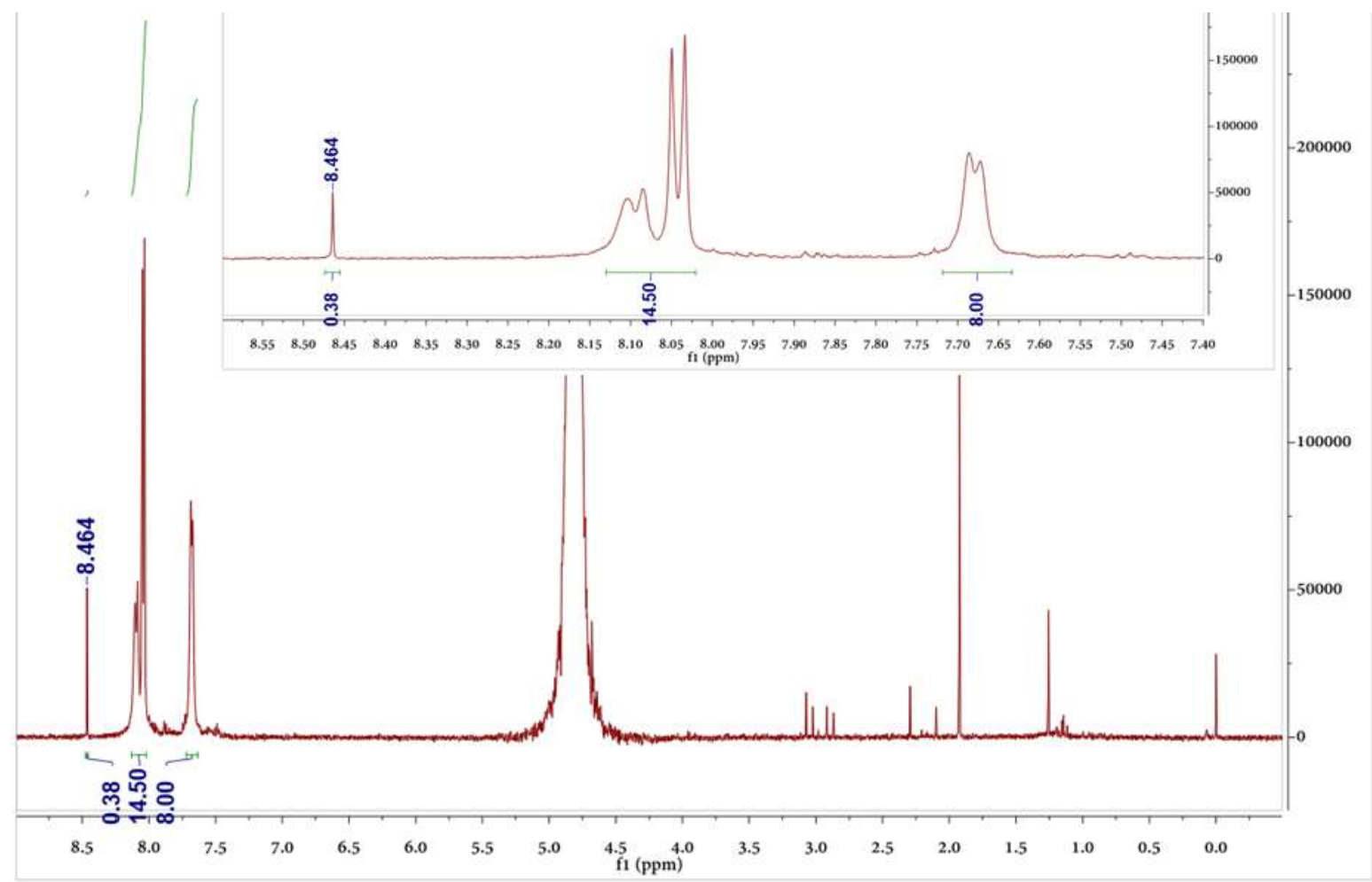

Figure S9. ${ }^{1} \mathrm{H}$ NMR spectrum of NU-1000-FF-Cl treated by $\mathrm{DMA} / \mathrm{HCl}$ at $100{ }^{\circ} \mathrm{C}$. The sample was digested in $0.1 \mathrm{M} \mathrm{NaOD} / \mathrm{D}_{2} \mathrm{O}$ and diluted in $\mathrm{D}_{2} \mathrm{O}$. There is no detectable benzoate, but there are 0.76 formate (at $8.464 \mathrm{ppm}$ ) ions per $\mathrm{Zr}_{6}$ node.

a)

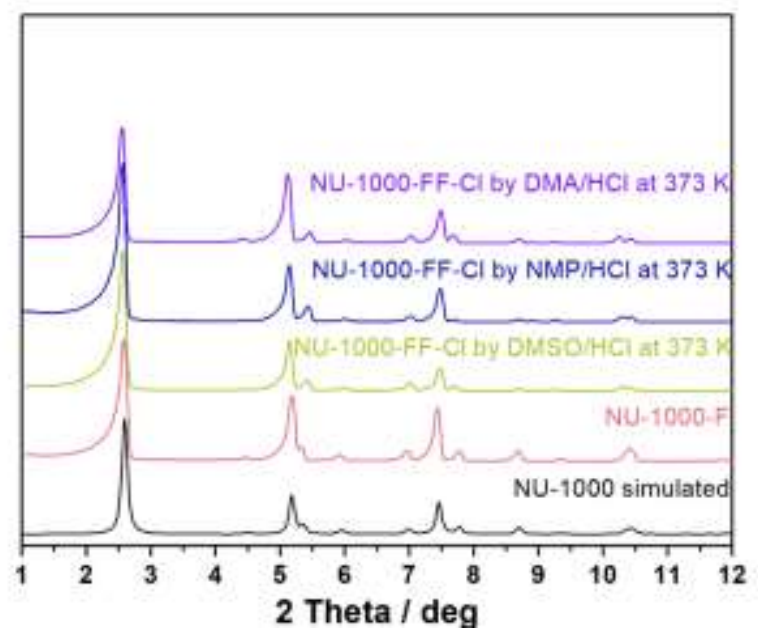

b)

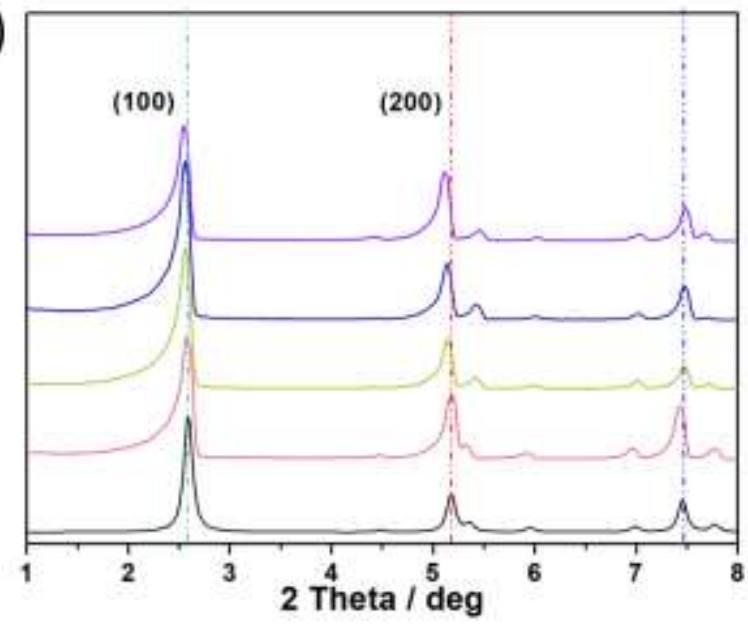

Figure S10. a) PXRD patterns of NU-1000-F and NU-1000-FF-Cl samples treated by different kinds of non-formate-generating solvent: DMSO (violet), NMP (navy), and DMA (red); b) amplified version of a) showing the range between $1^{\circ}$ to $8^{\circ}$. 

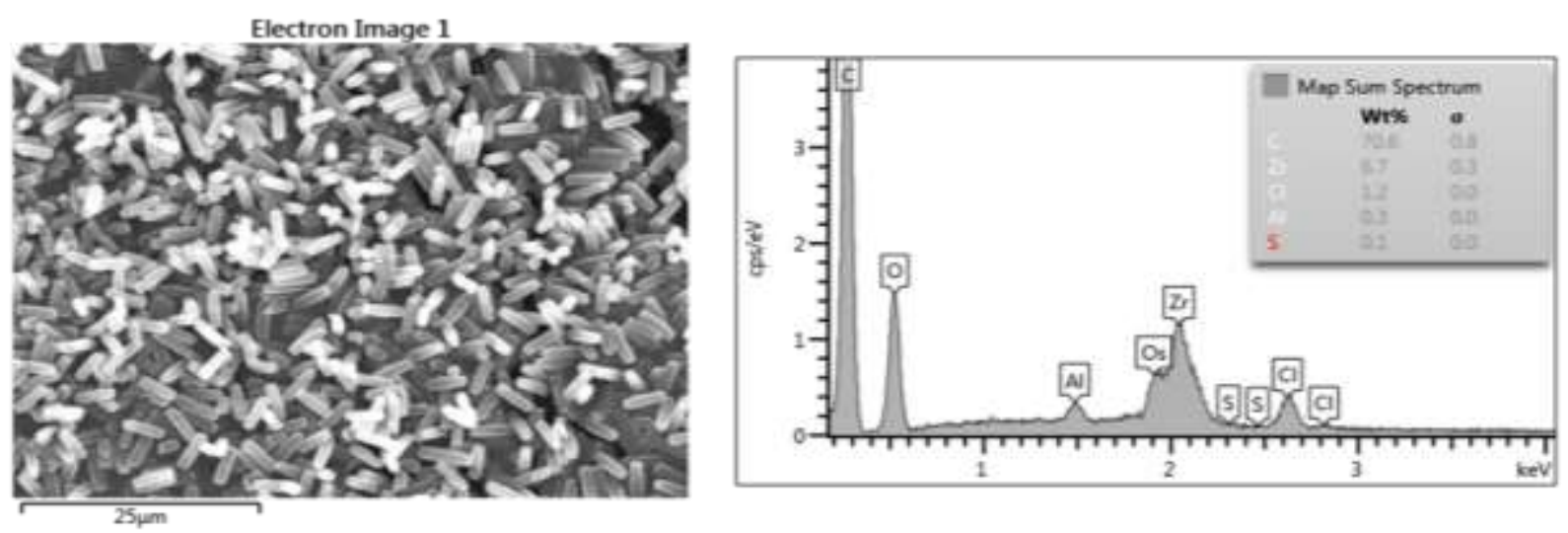

Figure S11. SEM image and EDS elemental mapping analysis of NU-1000-FF-Cl treated by $\mathrm{DMSO} / \mathrm{HCl}$ at $373 \mathrm{~K}$.
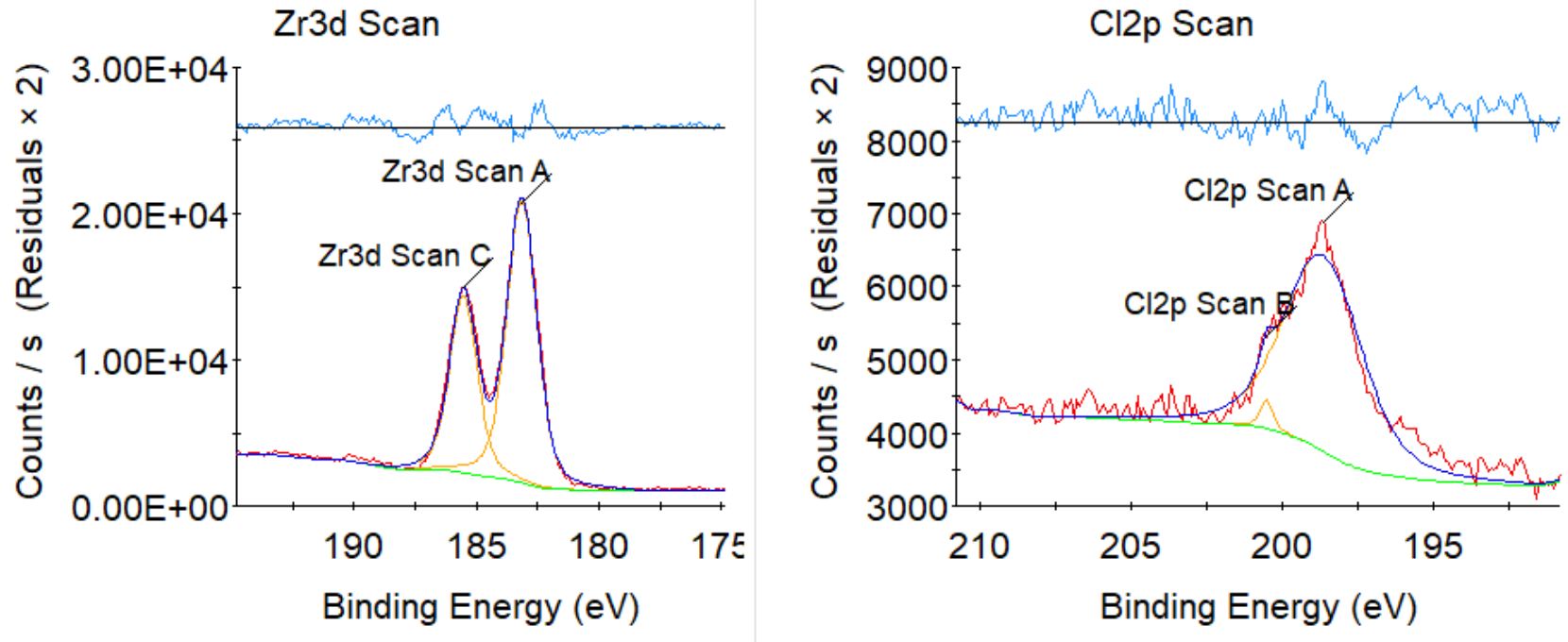

Figure S12. XPS of NU-1000-FF-Cl sample treated by $\mathrm{DMSO} / \mathrm{HCl}$ at $373 \mathrm{~K}$. The $\mathrm{Cl}: \mathrm{Zr}_{6}$ ratio was found to be 3.7:1 from the integrated peak areas of the $\mathrm{Cl} 2 \mathrm{p}$ and $\mathrm{Zr} 3 \mathrm{~d}$ core levels. 


\section{Access to NU-1000-FF-Cl at Room Temperature}
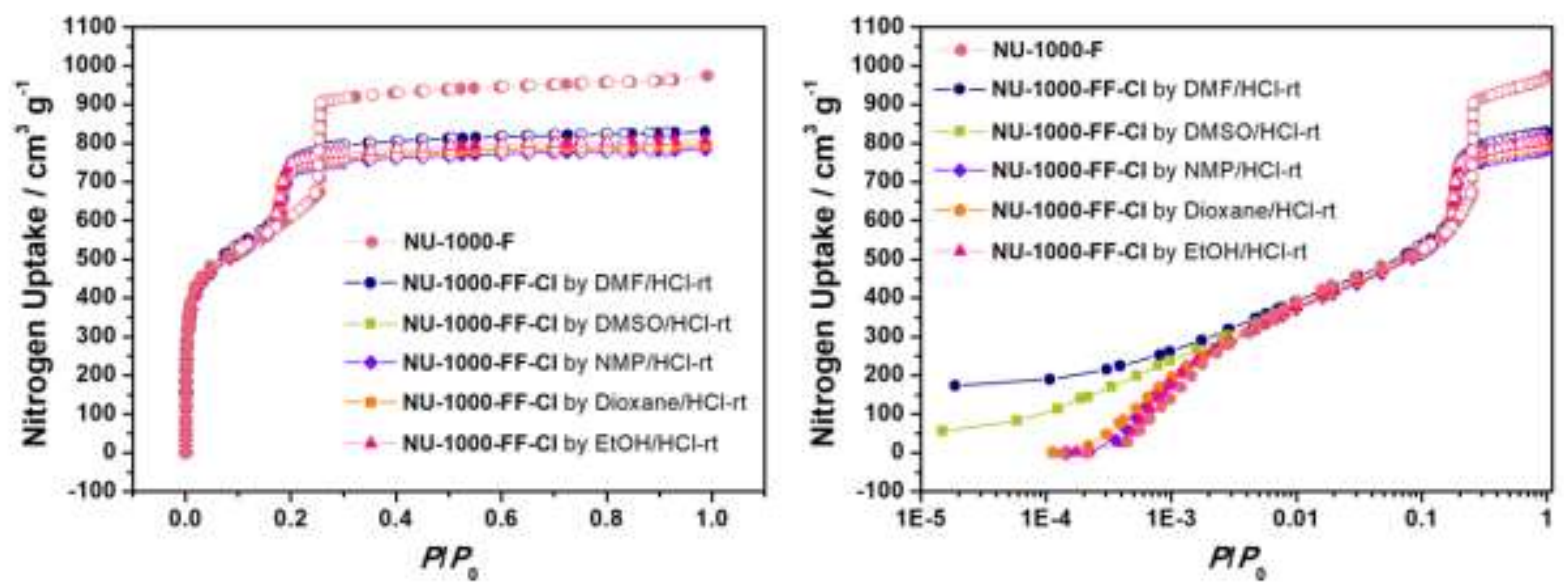

Figure S13. $\mathrm{N}_{2}$ adsorption isotherms at $77 \mathrm{~K}$ for NU-1000-F (kermesinus hexagon), and NU-1000-FFCl samples treated by different kinds of solvent such as DMF (navy circle), DMSO (pink square), NMP (violet diamond), Dioxane (orange pentagon), and Ethanol (green triangle). Left: linear $\mathrm{P} / \mathrm{P}_{0}$ scale; right: logarithmic $\mathrm{P} / \mathrm{P}_{0}$ scale.

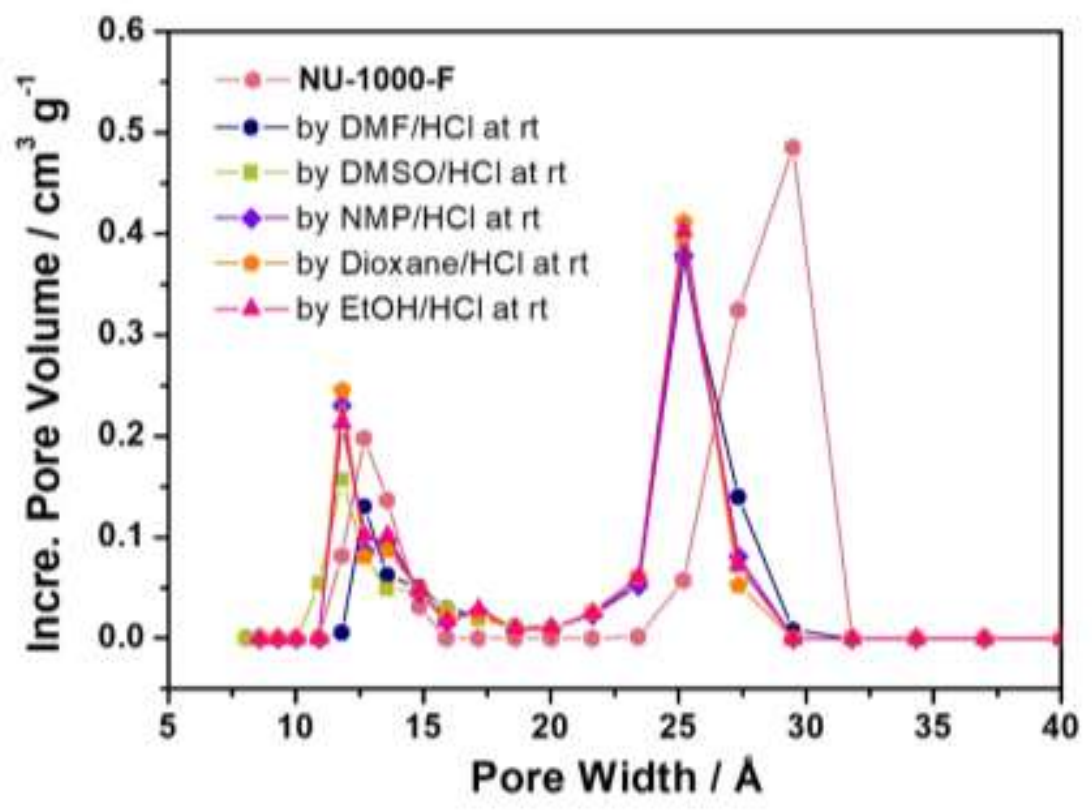

Figure S14. Pore size distributions of NU-1000-F (kermesinus hexagon), and NU-1000-FF-Cl samples treated by different kinds of solvent such as DMF (navy circle), DMSO (pink square), NMP (violet diamond), Dioxane (orange pentagon), and Ethanol (green triangle). 


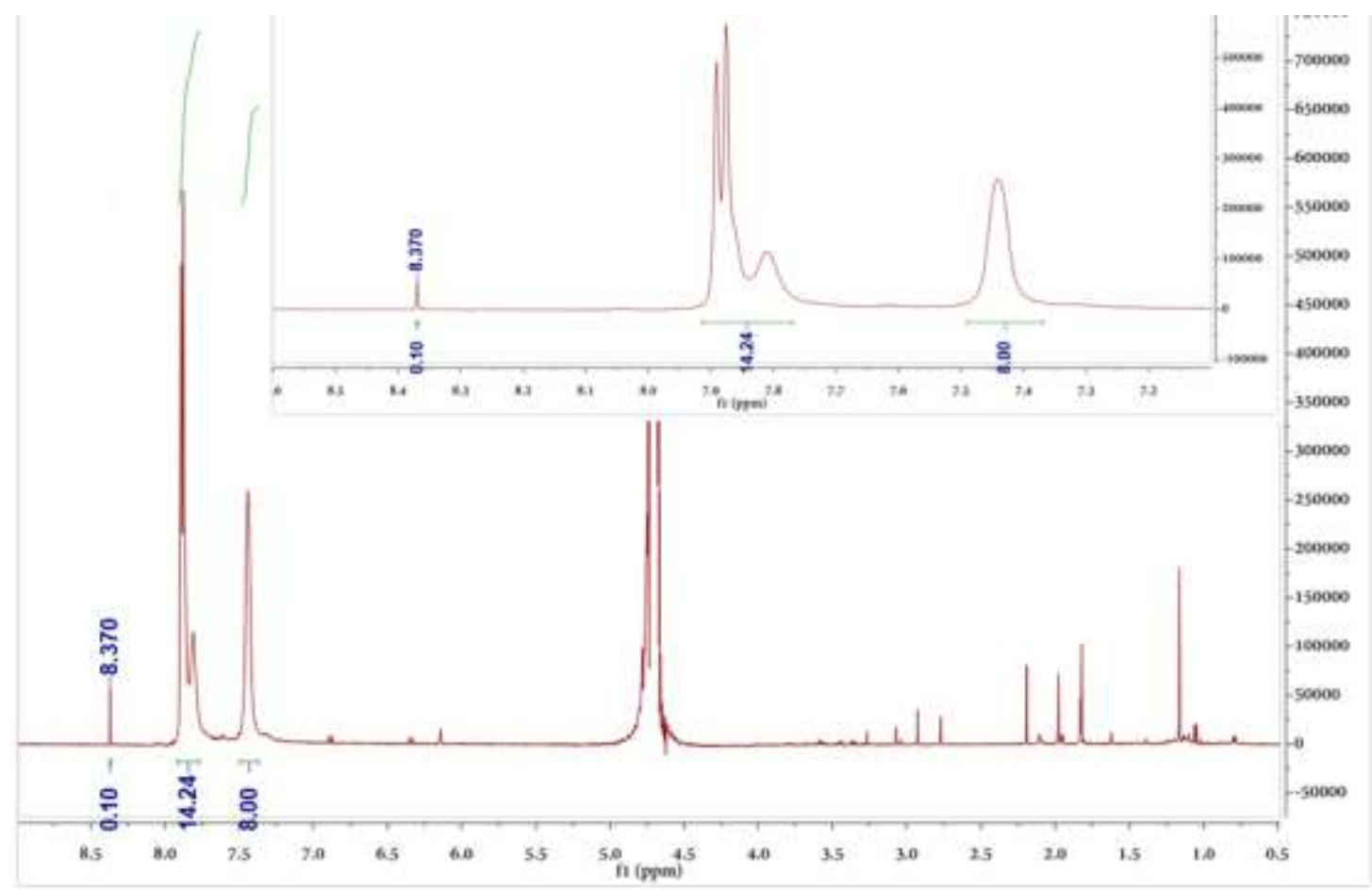

Figure S15. ${ }^{1} \mathrm{H}$ NMR spectrum of NU-1000-FF-Cl treated by $\mathrm{DMF} / \mathrm{HCl}$ at room temperature. The sample was digested in $0.1 \mathrm{M} \mathrm{NaOD} / \mathrm{D}_{2} \mathrm{O}$ and diluted in $\mathrm{D}_{2} \mathrm{O}$. There is no benzoate ligand but 0.2 formate per $\mathrm{Zr}_{6}$ node.

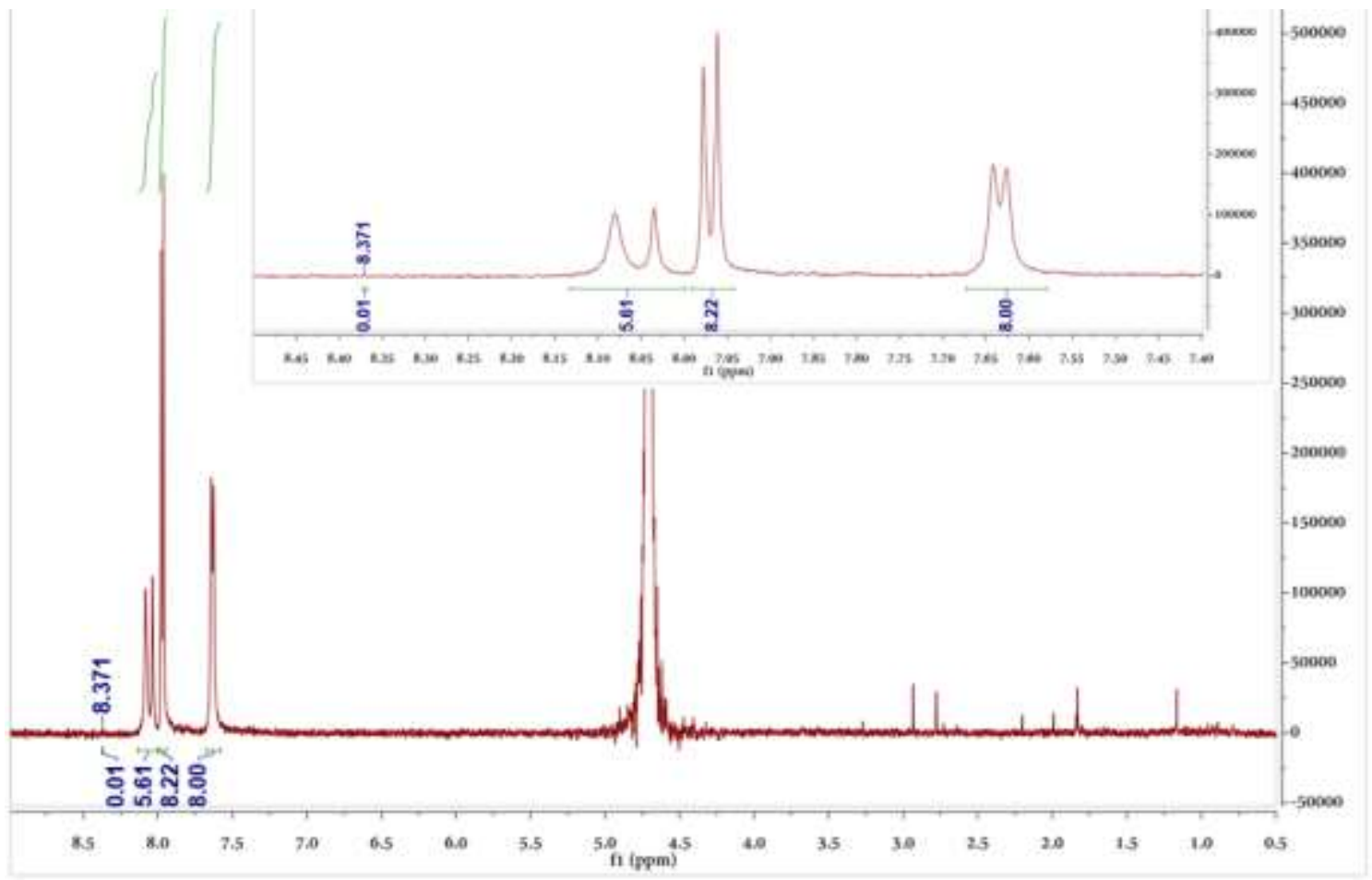

Figure S16. ${ }^{1} \mathrm{H}$ NMR spectrum of NU-1000-FF-Cl treated by DMSO/HCl at room temperature. The sample was digested in $0.1 \mathrm{M} \mathrm{NaOD} / \mathrm{D}_{2} \mathrm{O}$ and diluted in $\mathrm{D}_{2} \mathrm{O}$. There are no detectable benzoate or formate (at $8.371 \mathrm{ppm}$ ) ions. 


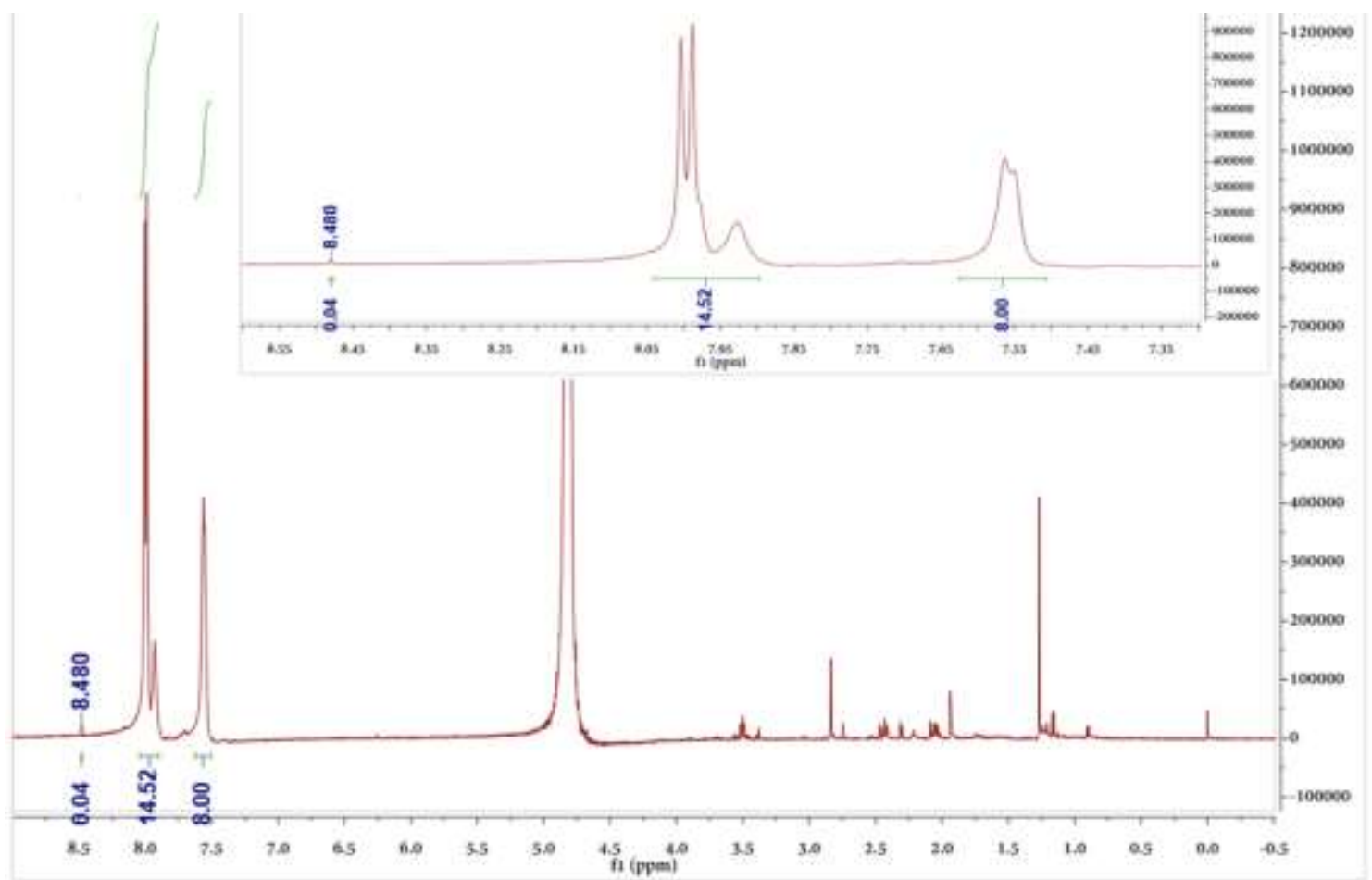

Figure S17. ${ }^{1} \mathrm{H}$ NMR spectrum of NU-1000-FF-Cl treated by $\mathrm{NMP} / \mathrm{HCl}$ at room temperature. The sample was digested in $0.1 \mathrm{M} \mathrm{NaOD} / \mathrm{D}_{2} \mathrm{O}$ and diluted in $\mathrm{D}_{2} \mathrm{O}$. There is no detectable benzoate and negligible formate ( 0.08 formate per $\mathrm{Zr}_{6}$ node, at $\left.8.480 \mathrm{ppm}\right)$.

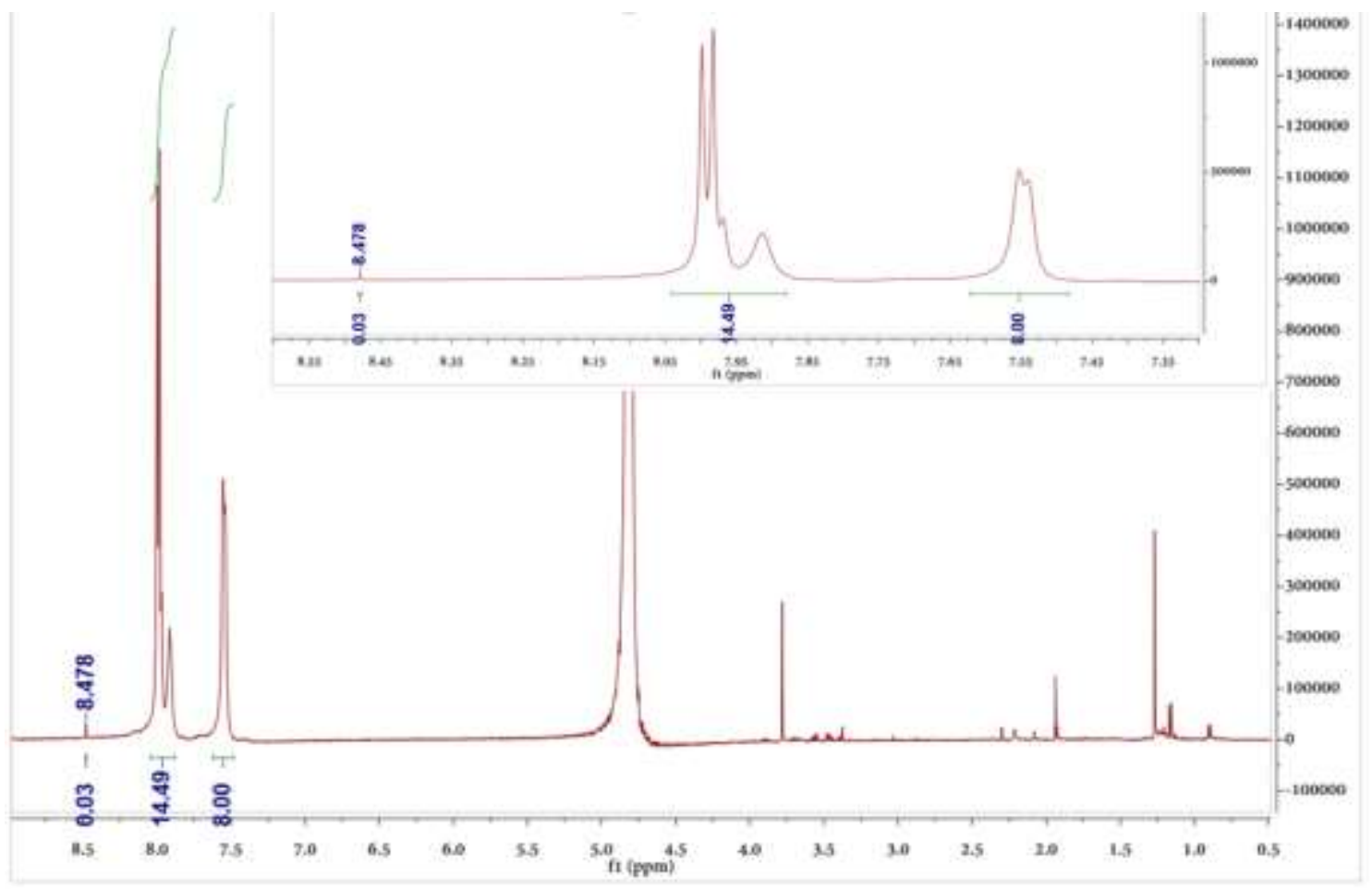

Figure S18. ${ }^{1} \mathrm{H}$ NMR spectrum of NU-1000-FF-Cl treated by Dioxane/HCl at room temperature. The sample was digested in $0.1 \mathrm{M} \mathrm{NaOD} / \mathrm{D}_{2} \mathrm{O}$ and diluted in $\mathrm{D}_{2} \mathrm{O}$. There is no detectable benzoate ligand and negligible formate ( 0.06 formate per $\mathrm{Zr}_{6}$ node, at $\left.8.480 \mathrm{ppm}\right)$. 


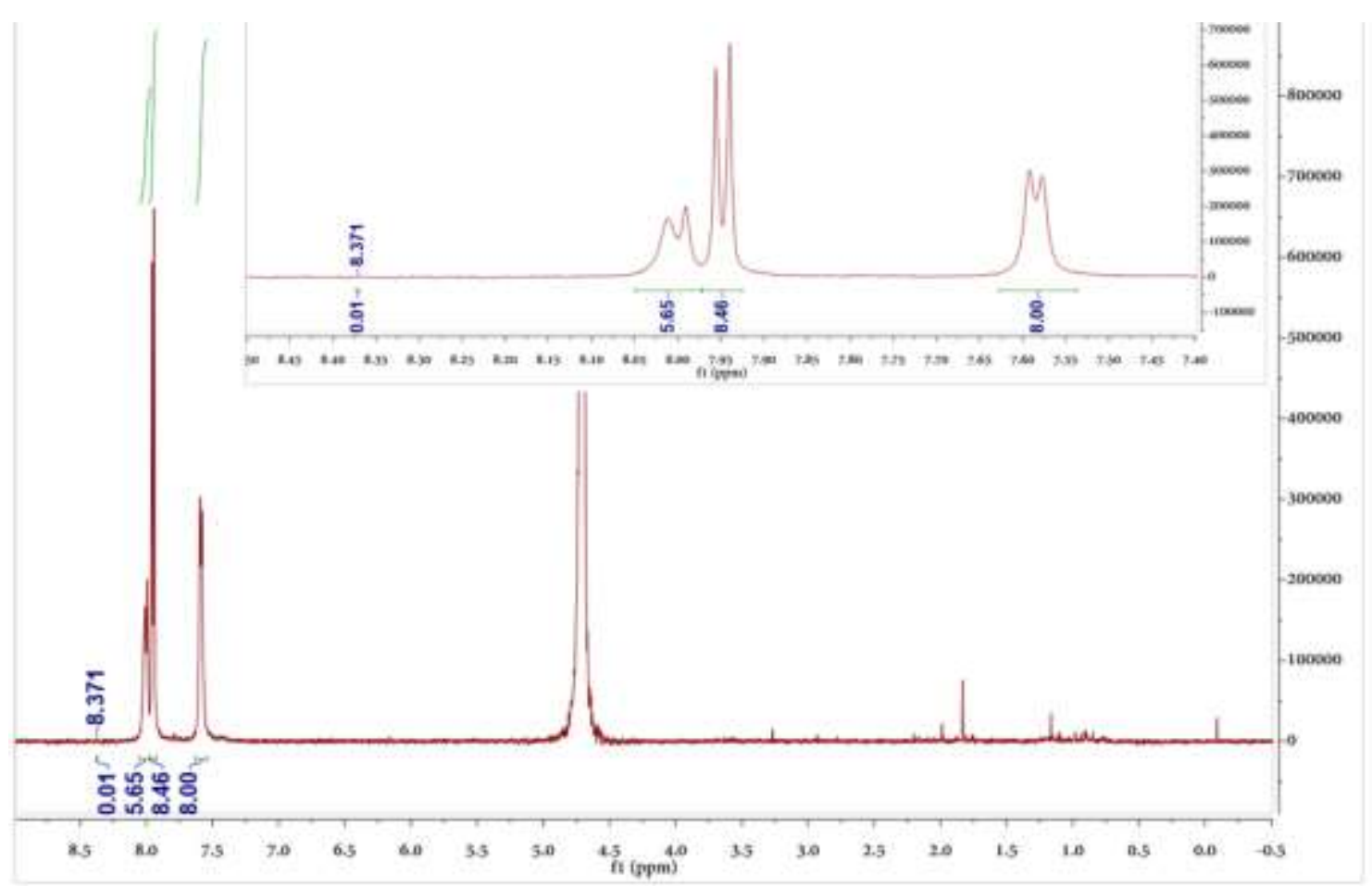

Figure S19. ${ }^{1} \mathrm{H}$ NMR spectrum of NU-1000-FF-Cl treated by Ethanol/HCl at room temperature. The sample was digested in $0.1 \mathrm{M} \mathrm{NaOD} / \mathrm{D}_{2} \mathrm{O}$ and diluted in $\mathrm{D}_{2} \mathrm{O}$. There are no detectable benzoate or formate (at $8.371 \mathrm{ppm})$ ions.

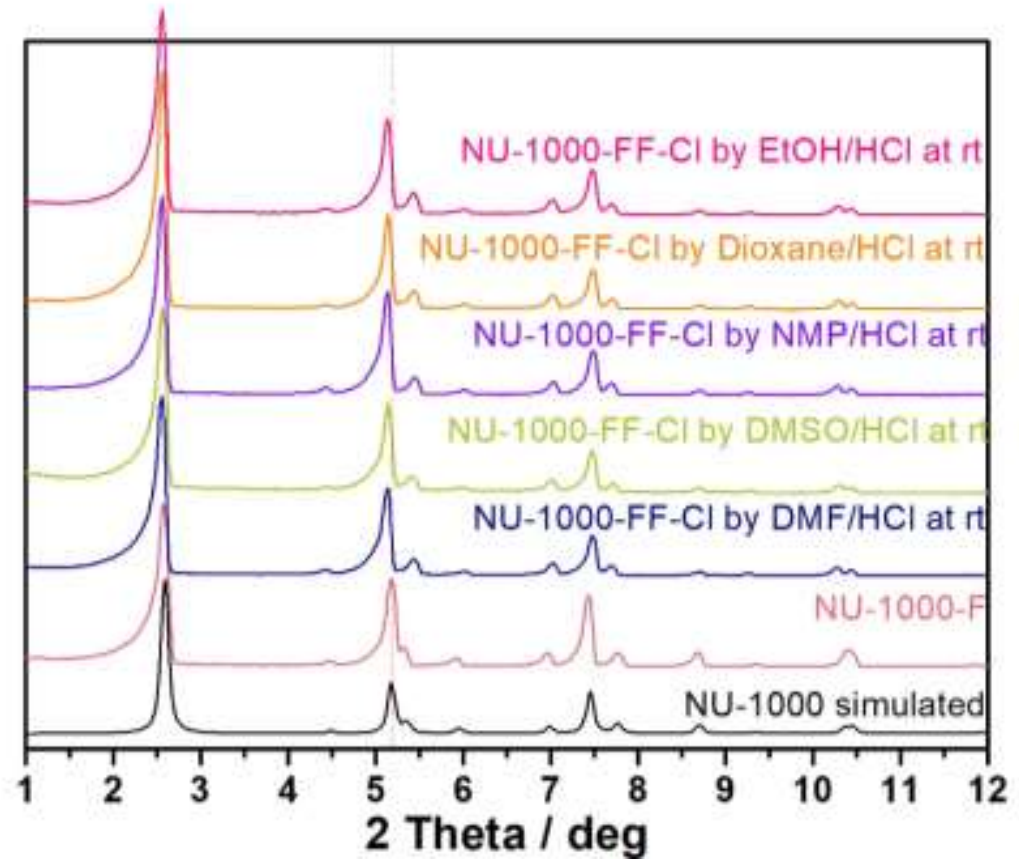

Figure S20. PXRD patterns of NU-1000-F and NU-1000-FF-Cl samples treated by different kinds of solvent at room temperature: DMF (navy), DMSO (pink), NMP (violet), Dioxane (orange), and Ethanol (green). 

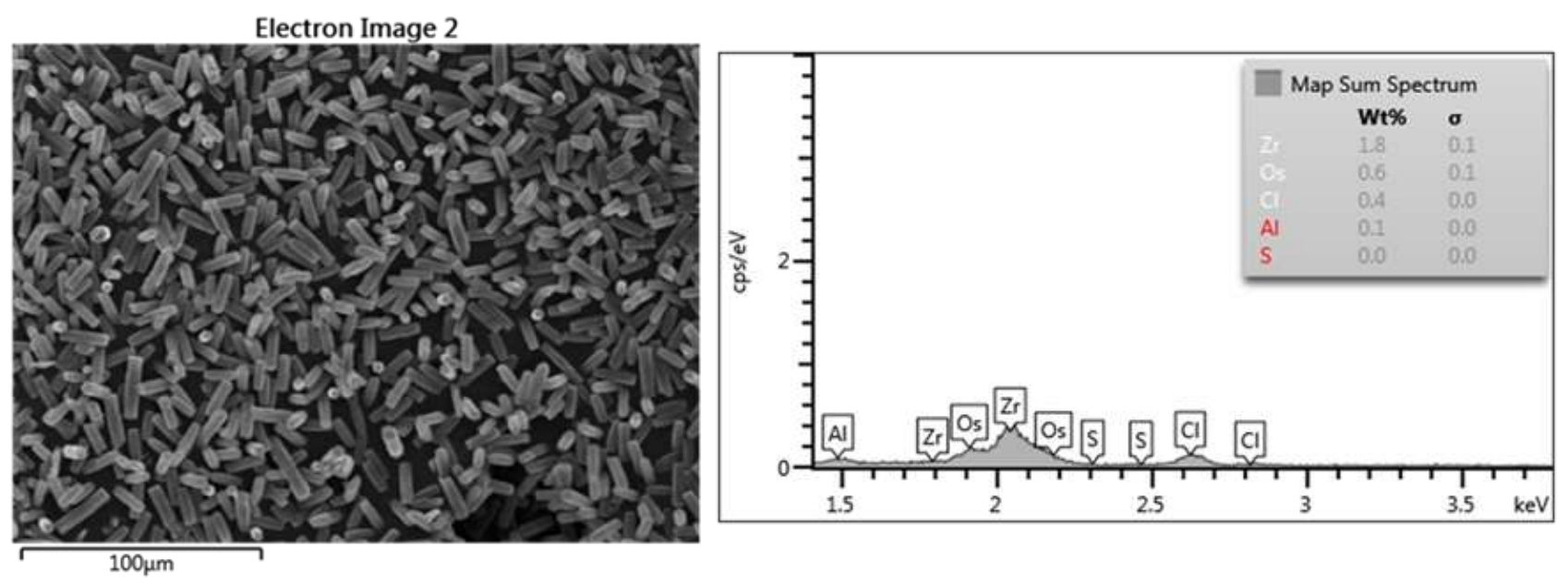

Figure S21. SEM image and EDS elemental mapping analysis of NU-1000-FF-Cl treated by DMF/HCl at room temperature.
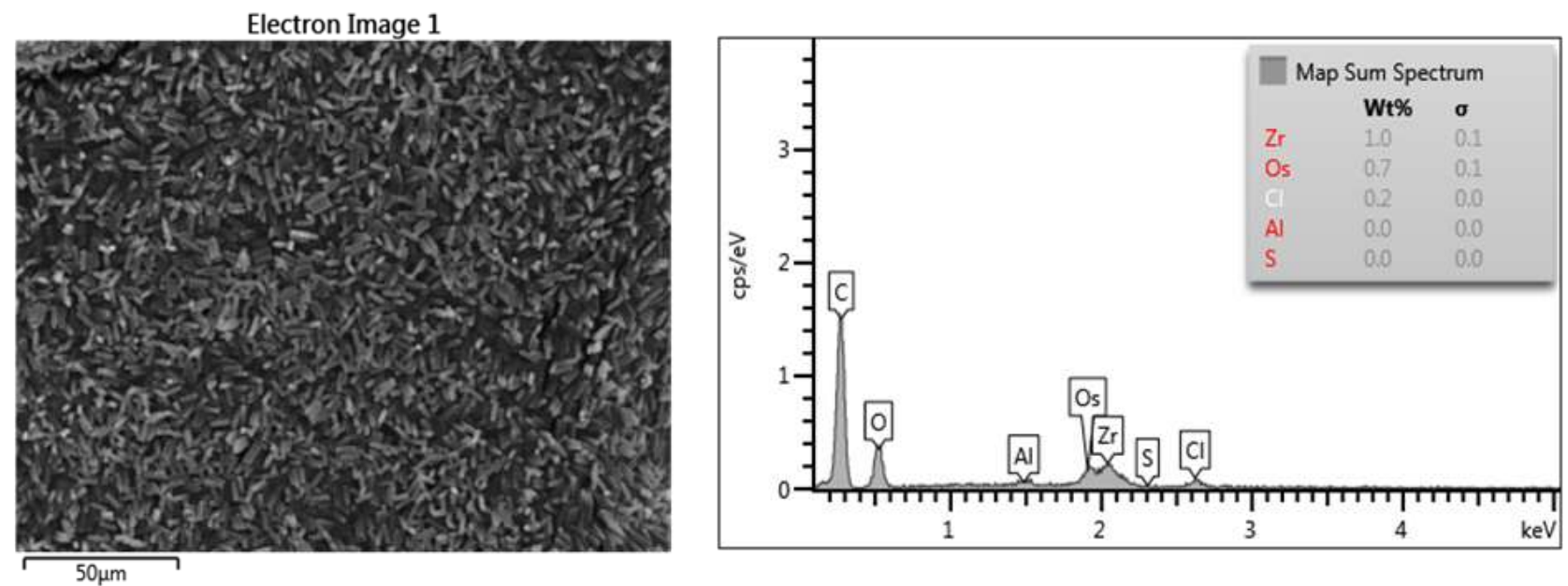

Figure S22. SEM image and EDS elemental mapping analysis of NU-1000-FF-Cl treated by NMP/HCl at room temperature. 
Table S1. Comparison between NU-1000-F and NU-1000-FF-CI treated by different methods: formate loading, chloride residue, and BET surface area.

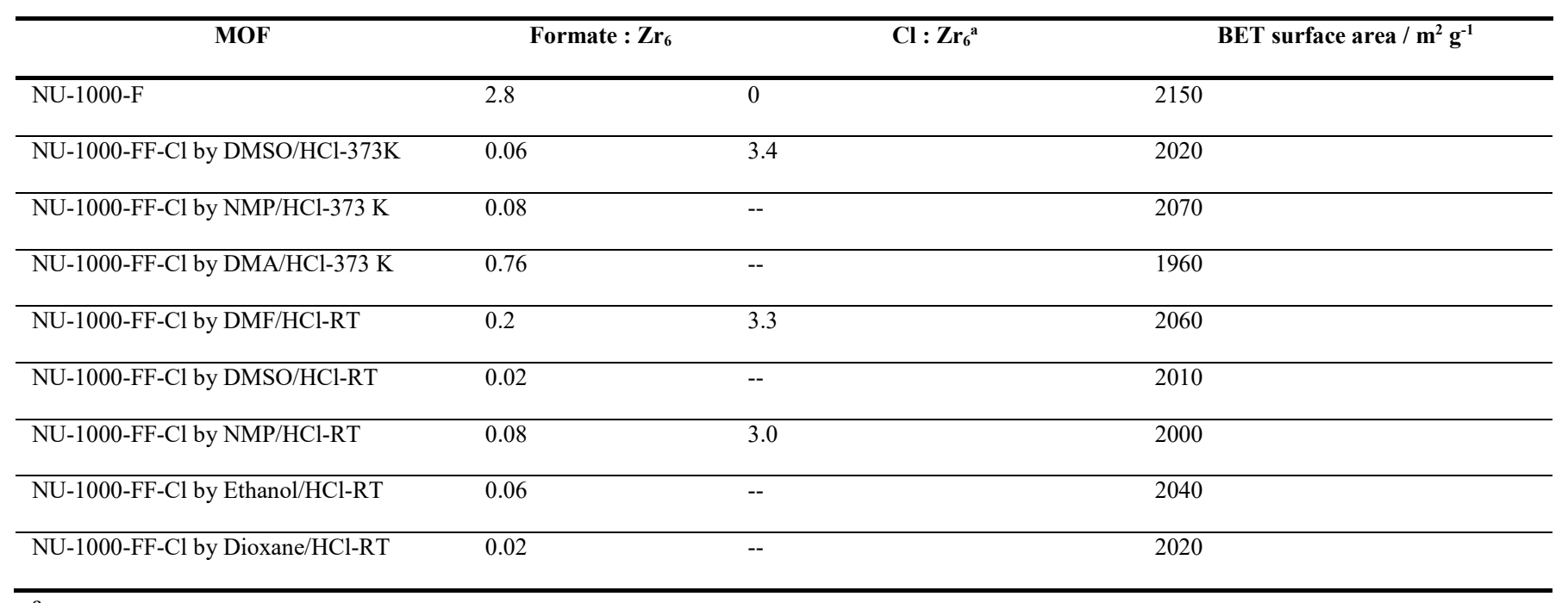

\footnotetext{
${ }^{a}$ data collected by SEM-EDS
}

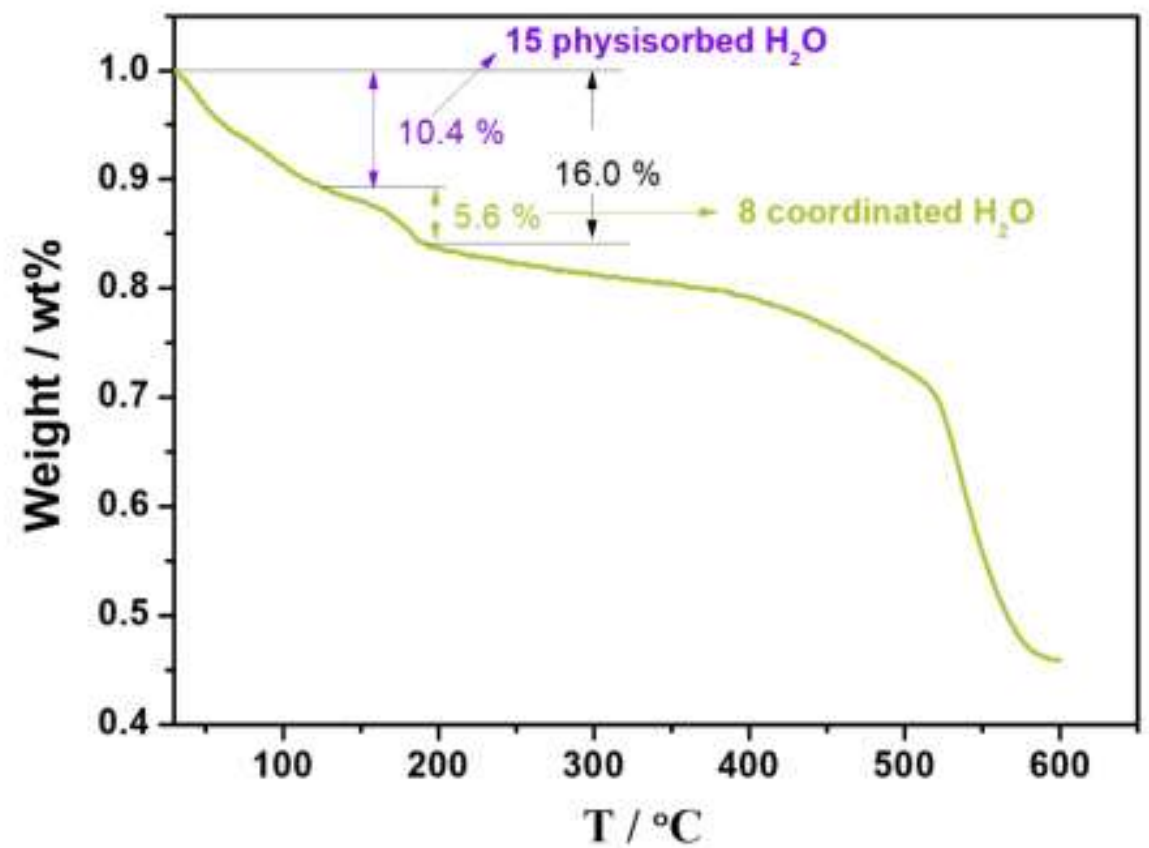

Figure S23. TG curve of NU-1000-FF-Cl indicating a loss of $\sim 8$ coordinated $\mathrm{H}_{2} \mathrm{O}$ at $125 \sim 175{ }^{\circ} \mathrm{C}$. 


\section{Single-crystal Data}

Single-crystal X-ray diffraction data of NU-1000-F and NU-1000-FF were measured on a Bruker Apex II CCD diffractometer at various temperatures using both graphite monochromated Mo/K $\alpha$ radiation $(\lambda=0.71073 \AA)$ and $\mathrm{Cu} / \mathrm{K} \alpha$ radiation $(\lambda=1.54178 \AA)$. Data reduction was made with the Bruker SAINT programs. Single-crystal X-ray diffraction data of NU-1000-FF-Cl, NU-1000-FF-Br, and NU-1000-FFheptane-exchanged were measured on Rigaku Oxford Diffraction XtaLAB Synergy-S at $100 \mathrm{~K}$ using graphite monochromated $\mathrm{Cu} / \mathrm{K} \alpha$ radiation $(\lambda=1.54184 \AA)$. Data reduction was made with the Rigaku CrysAlisPro programs. The structures were solved by direct (SHELXS) and intrinsic phasing (SHELXT) methods and refined with full-matrix least squares technique using the SHELXL package. Non-hydrogen atoms were refined with anisotropic displacement parameters during the final cycles. Organic hydrogen atoms were placed in calculated positions with isotropic displacement parameters set to $1.2 \times$ Ueq of the attached atom. The unit cell includes a large region of disordered solvent molecules, which could not be modeled as discrete atomic sites. We employed PLATON/SQUEEZE to calculate the diffraction contribution of the solvent molecules and thereby, to produce a set of solvent-free diffraction intensities; structures were then refined again using the data generated. Crystallographic data for the NU-1000-F, NU-1000-FF-CI, NU-1000-FF-heptane-exchanged, NU-1000-FF, and NU-1000-FF-Br, crystal structures in CIF format have been deposited in the Cambridge Crystallographic Data Centre (CCDC) under deposition numbers CCDC-2030766-2030770. The data can be obtained free of charge via www.ccdc.cam.ac.uk/data_request/cif (or from the Cambridge Crystallographic Data Centre, 12 Union Road, Cambridge CB2 1EZ, U.K.)

Table S2. Crystallographic Data

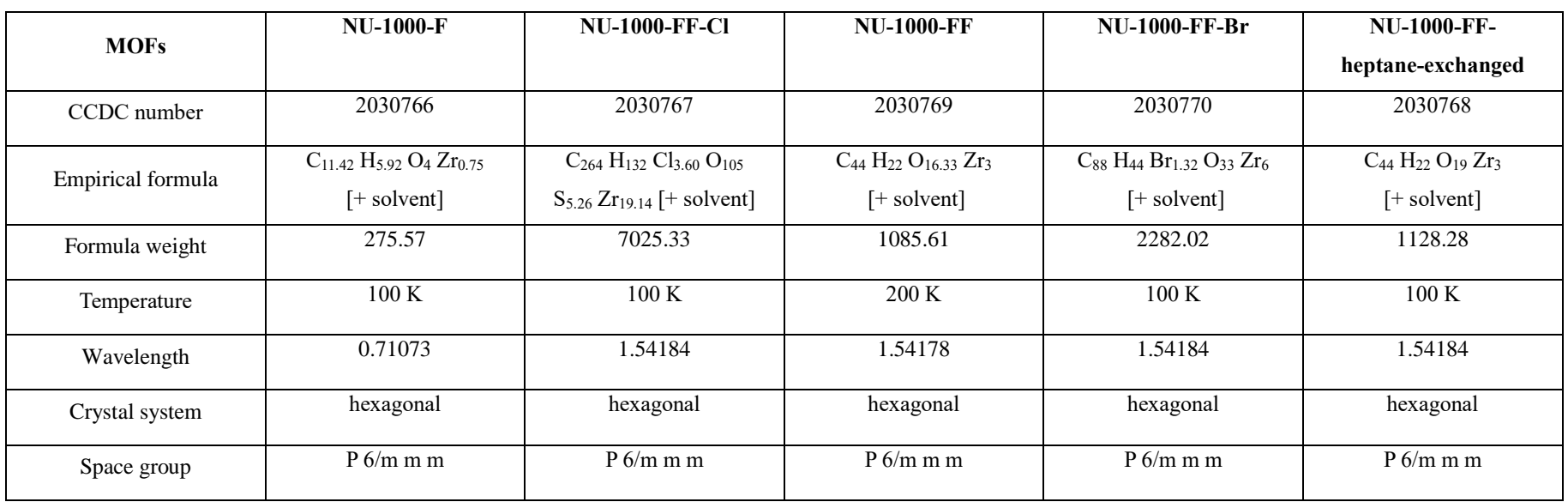




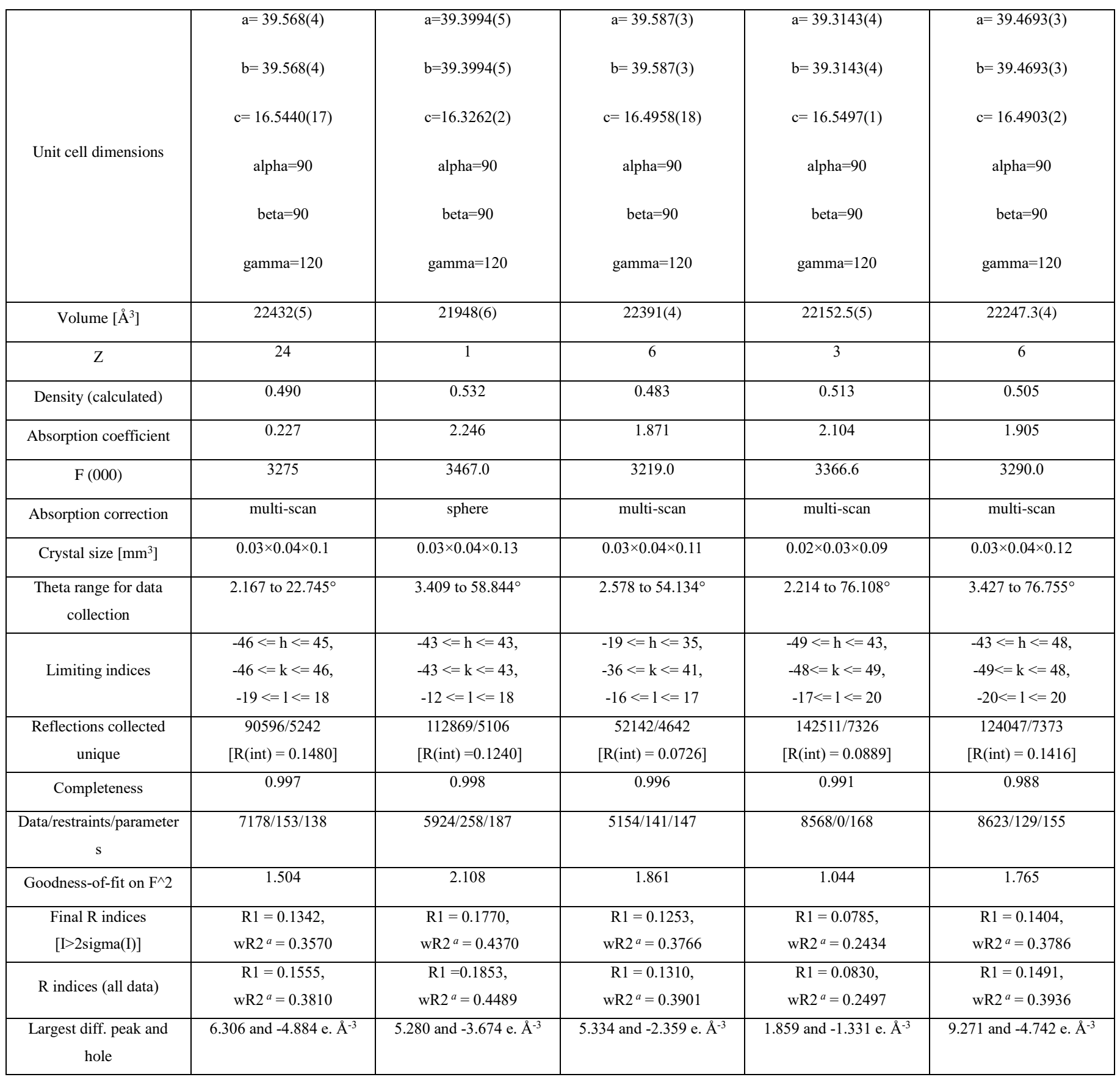

${ }^{a} \mathrm{R} 1=\Sigma|| F_{o}|-| F_{c}|| /\left|F_{o}\right| ; \mathrm{wR} 2=\left[\Sigma w\left(\Sigma F_{o}^{2}-F_{c}^{2}\right)^{2} / \Sigma w\left(F_{o}^{2}\right)^{2}\right]^{1 / 2}$. 

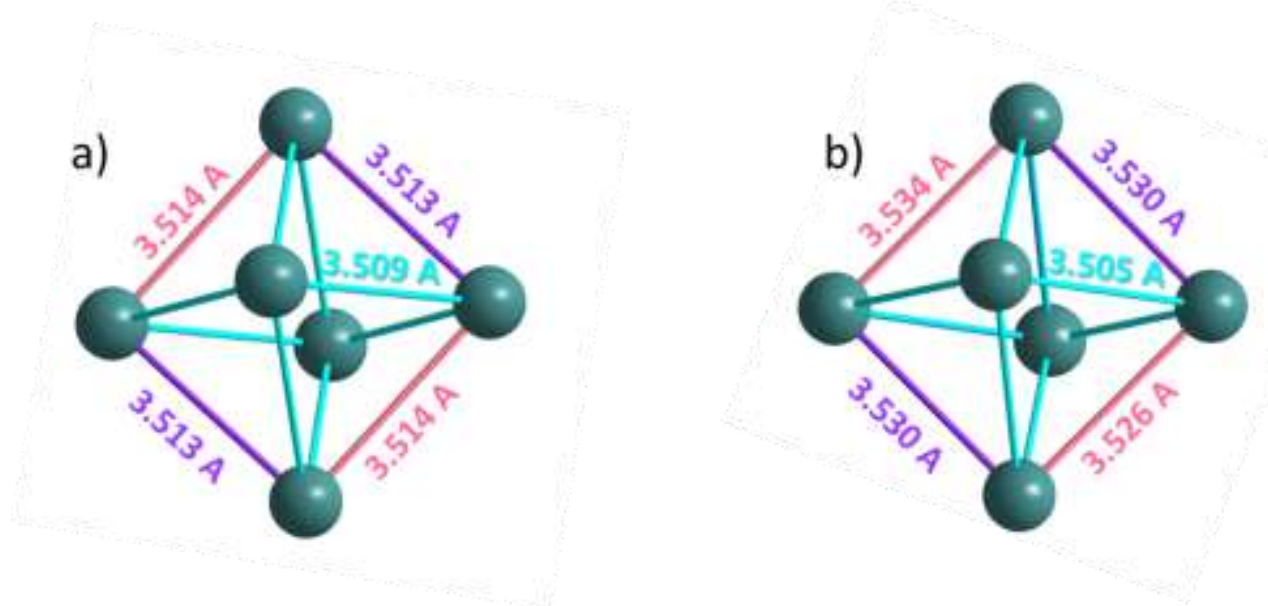

Figure S24. a) Zr6-node geometry of NU-1000-F; b) Zr6-node geometry of NU-1000-FF-Cl.

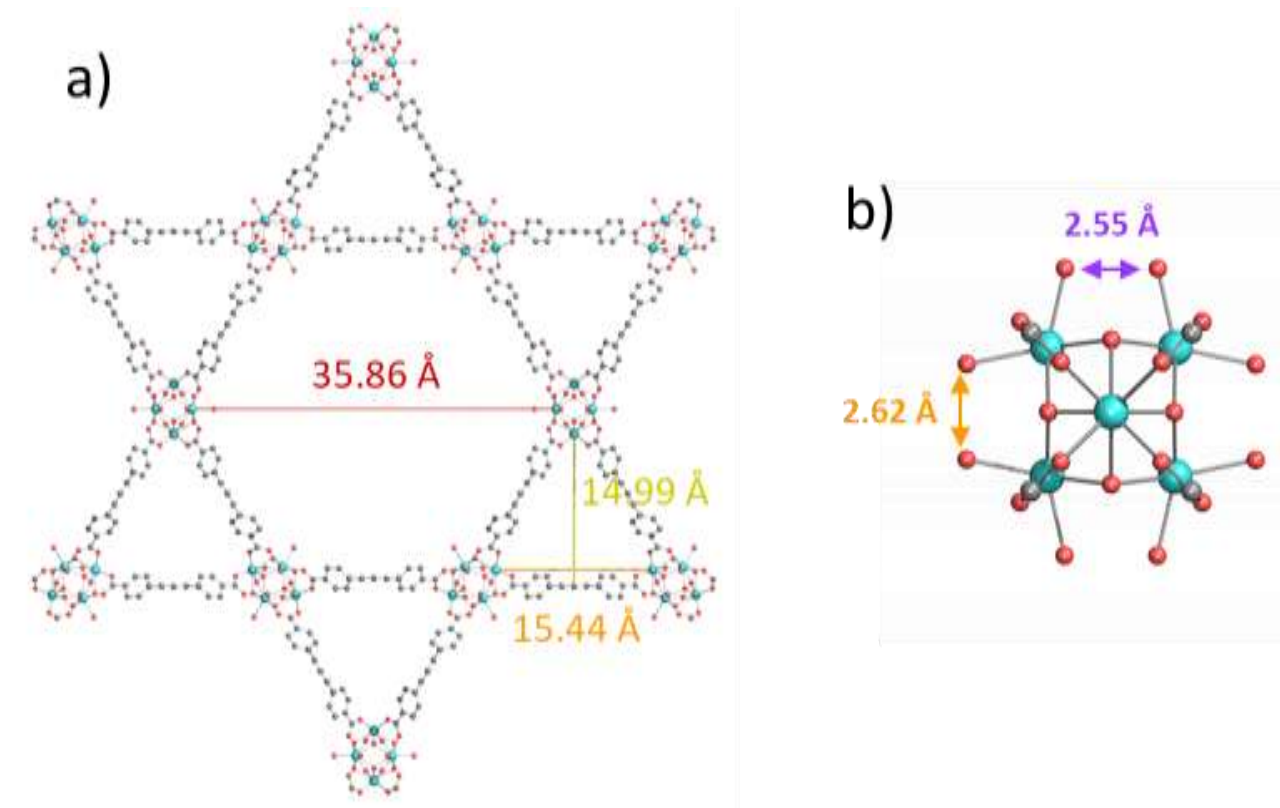

Figure S25. The crystal structure of NU-1000 previously reported. a) pore sizes; b) $\mathrm{Zr}_{6}$-node geometry

The positive and negative residual electron densities in the structures, which are located very close to the $\mu_{3}$-oxygen atoms and at the center of the Zr-oxo cluster (Figure S26, 27 and 29), are due to the disorder of the $\mu_{3}$-oxygen atoms and the diffuse scattering of the $\mathrm{Zr}$-oxo cluster.

We found that both NU-1000-FF-Cl and NU-1000-FF contains q peaks close to the terminal oxygen atoms (located on their middle-plane with distances of about $3 \AA$ ) of the node. Since in NU-1000-FF chloride has already been removed, these q peaks are likely to be oxygen atoms from water. In order to 
confirm this, we soaked NU-1000-FF single-crystals in anhydrous heptane in a glovebox, refreshing the solvent periodically, to obtain NU-1000-FF-heptane-exchanged single crystals. In the structure of NU1000-FF-heptane-exchanged, half of these $q$ peaks disappear, which indicates that these $q$ peaks are water molecules that can be removed by solvent-exchange.

It was not straightforward to assign the q peaks close to the terminal oxygen atoms of the node of NU1000-FF-Cl, as partially occupied oxygen atoms (from water) and partially occupied (lower occupancy than the alternatively assigned oxygen) chloride ions are difficult to distinguish. Since the $q$ peaks mentioned above (for NU-1000-FF) are believed to be water molecules, in order to further identify the location of chloride ions in NU-1000-FF-Cl, we prepared NU-1000-FF-Br single crystals. We did this by soaking NU-1000-F single crystals in a solution of DMSO + aq. $\mathrm{HBr}$ at room temperature. We found that residual electron density of the same location as mentioned above, is higher than expected for a fully occupied oxygen atom (Figure S28), implying the presence of partially occupied bromide ion instead of water close to the terminal oxygen atoms of the nodes. Therefore, the q peak close to the terminal oxygen of NU-1000-FF-Cl node was assigned as partially occupied chloride instead of water (Figure S29).

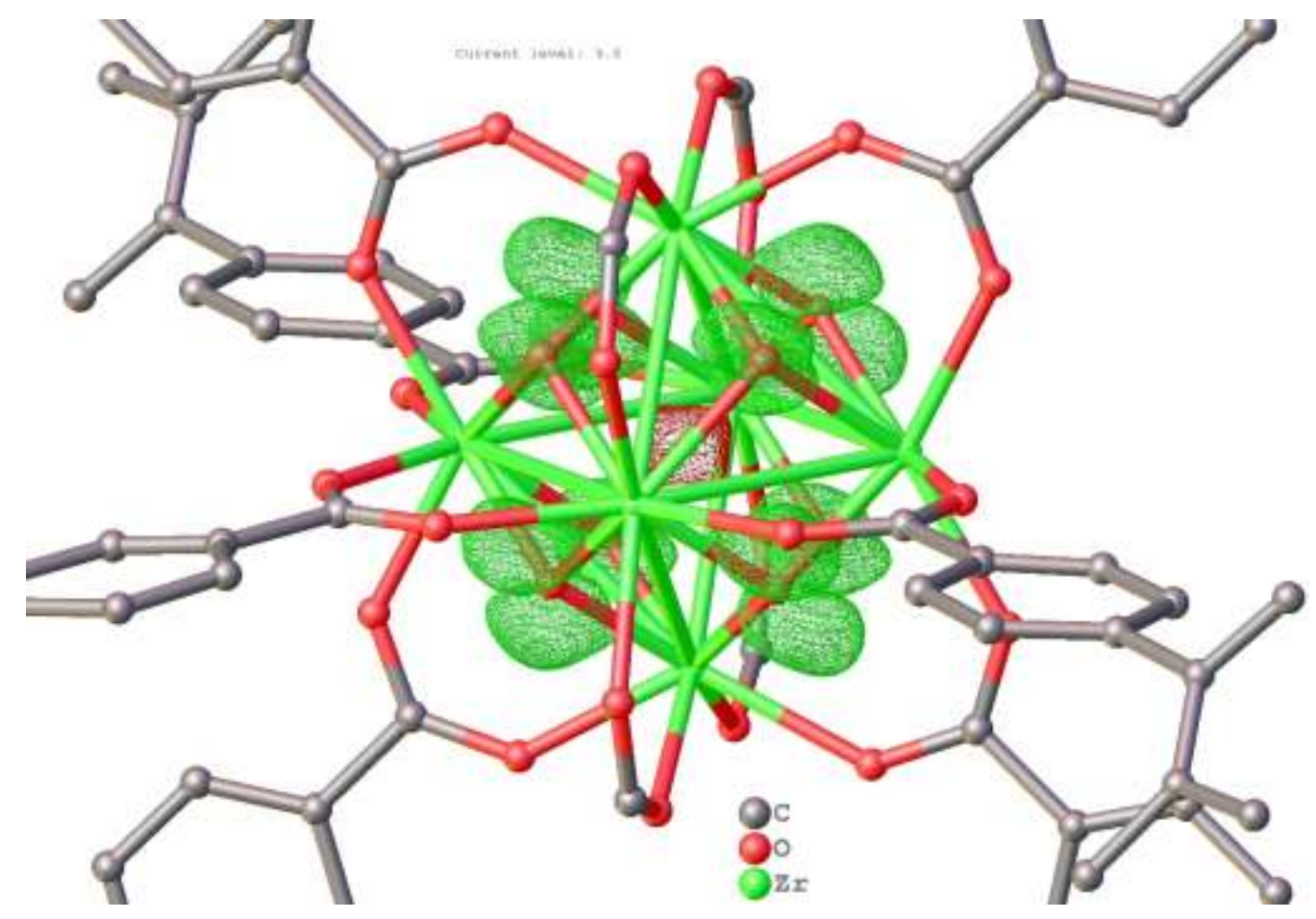

Figure S26. Difference electron density map of NU-1000-F showing that the large positive (green mesh) and negative (red mesh) residual density are due to the disorder of the $\mu_{3}-\mathrm{O}$ and/or diffuse scattering of the $\mathrm{Zr}$ node. 


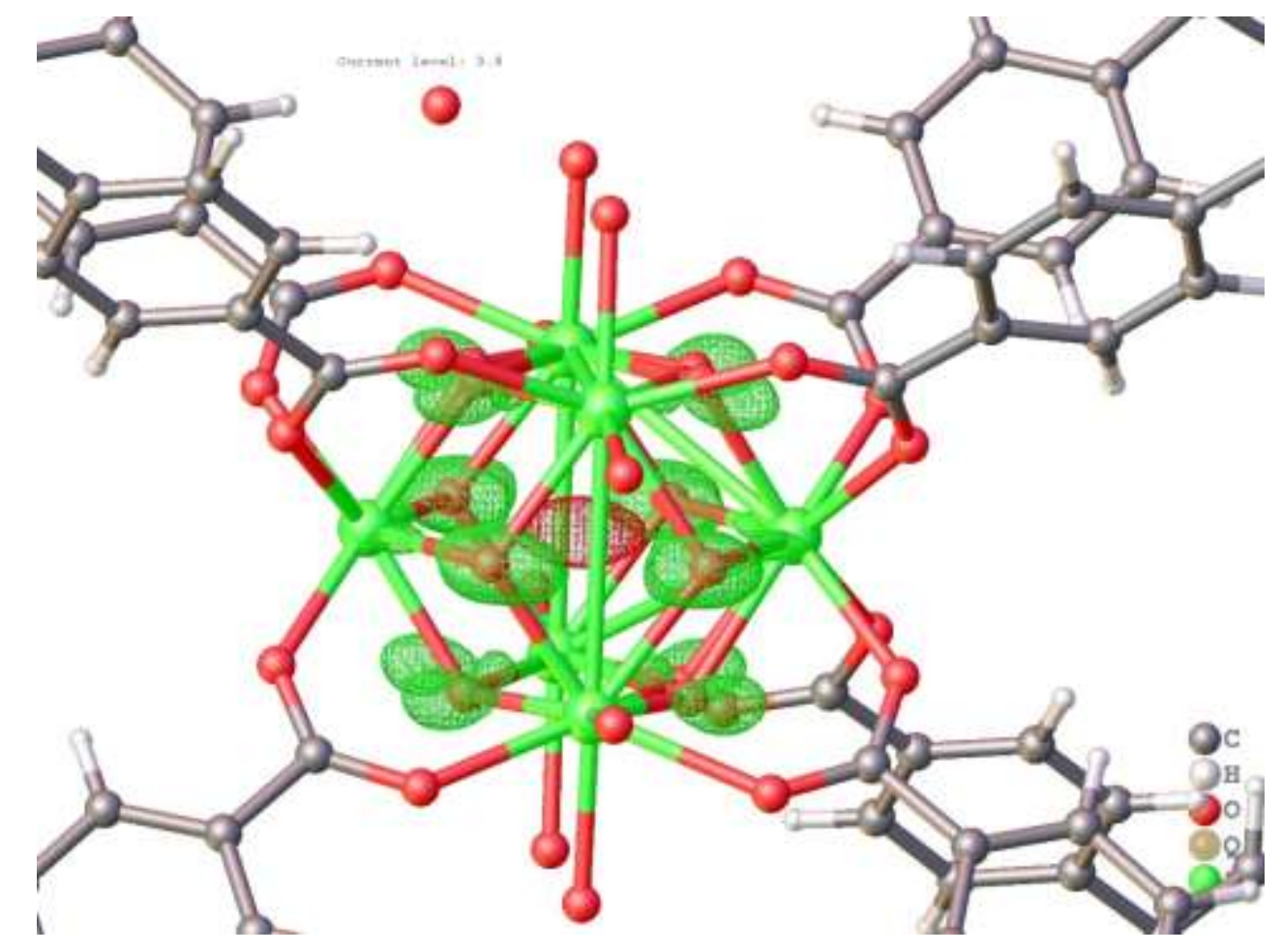

Figure S27. Difference electron density map of NU-1000-FF-CIF-Heptane showing that the large positive (green mesh) and negative (red mesh) residual densities are due to disorder of the $\mu_{3}-\mathrm{O}$ and/or diffuse scattering of the $\mathrm{Zr}$ node.

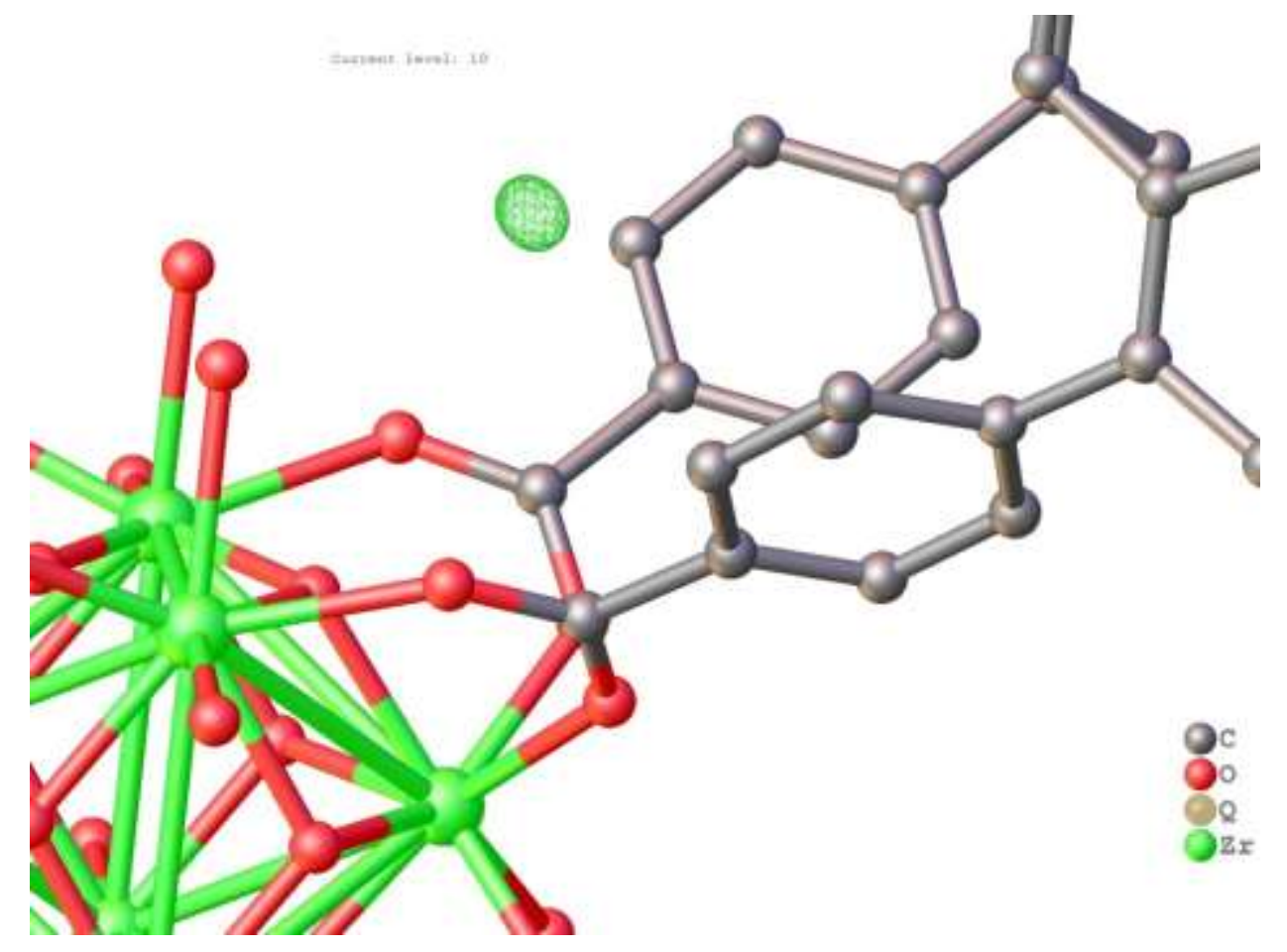

Figure S28. Difference electron density map of NU-1000-FF-Br showing that the large positive (green mesh) residual density close to the terminal oxygen atoms of the $\mathrm{Zr}$-node. As a result, this electron density was assigned to partially occupied $\mathrm{Br}$. 


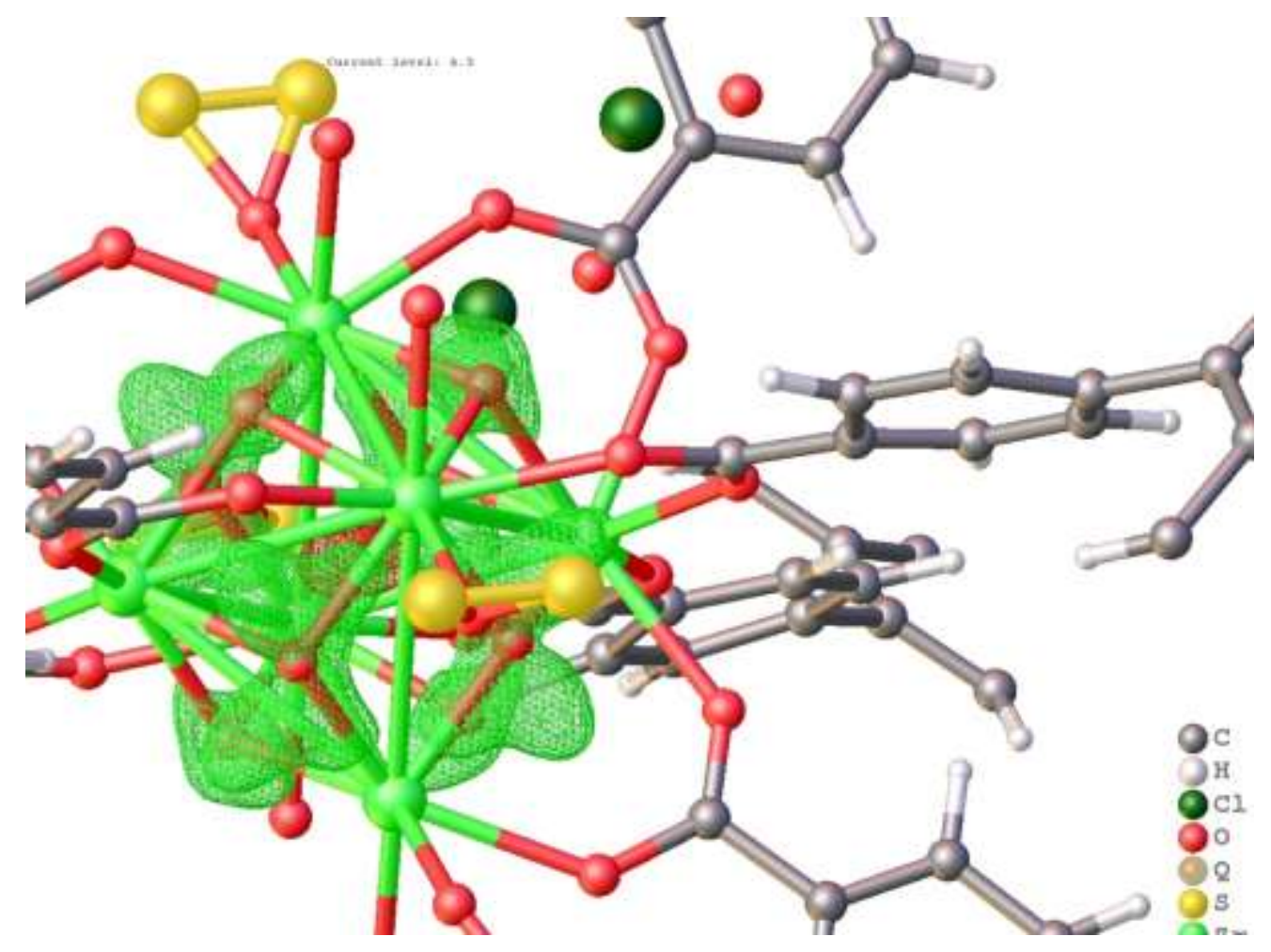

Figure S29. Difference electron density map of NU-1000-FF-Cl showing that that the large positive (green mesh) and negative (red mesh) residual densities are due to the disorder of the $\mu_{3}-\mathrm{O}$ and/or diffuse scattering of the $\mathrm{Zr}$ node. Note that there are partially resolved partially occupied DMSO molecules assigned to coordinate to the $\mathrm{Zr}$ atoms in the node. The carbon atoms on the DMSO molecules are not observed, which is likely due to low occupancy and disorder. 
6. NU-1000-FF-Cl Changing Back to NU-1000-F (denoted as R1-NU-1000F)
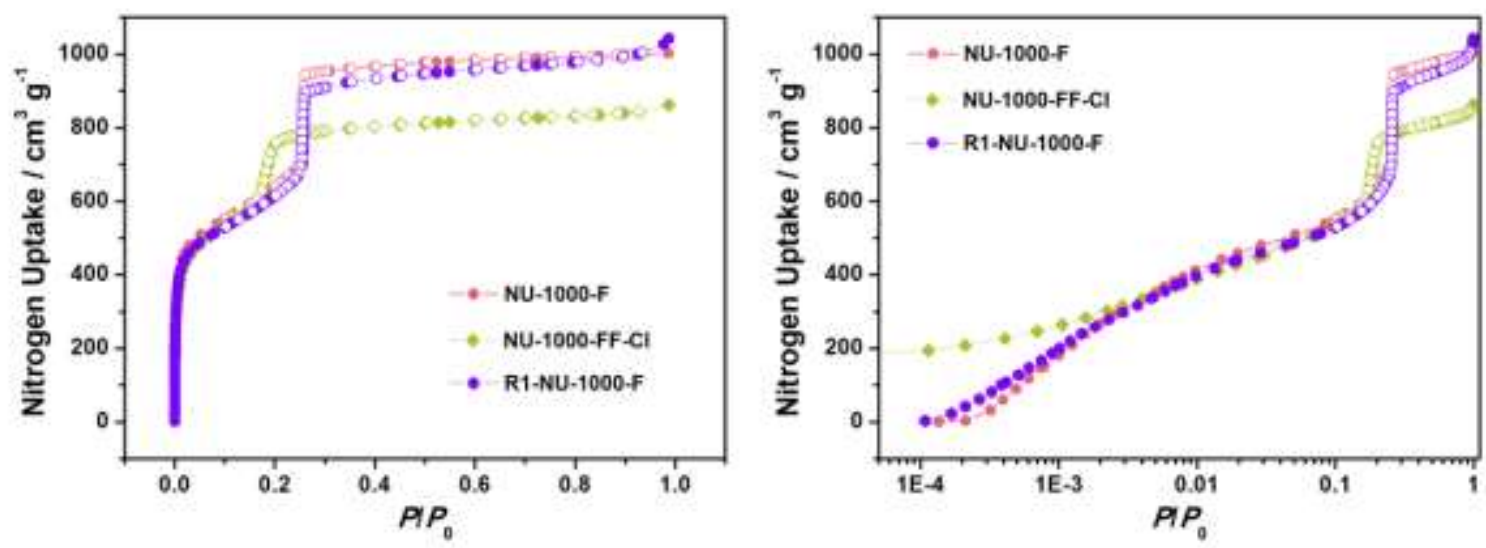

Figure S30. $\mathrm{N}_{2}$ adsorption isotherms at $77 \mathrm{~K}$ for NU-1000 (navy circle), and FF-NU-1000 (pink diamond) and NU-1000 regenerated from FF-NU-1000 (violet hexagon). Left: linear $\mathrm{P} / \mathrm{P}_{0}$ scale; right: logarithmic $\mathrm{P} / \mathrm{P}_{0}$ scale.

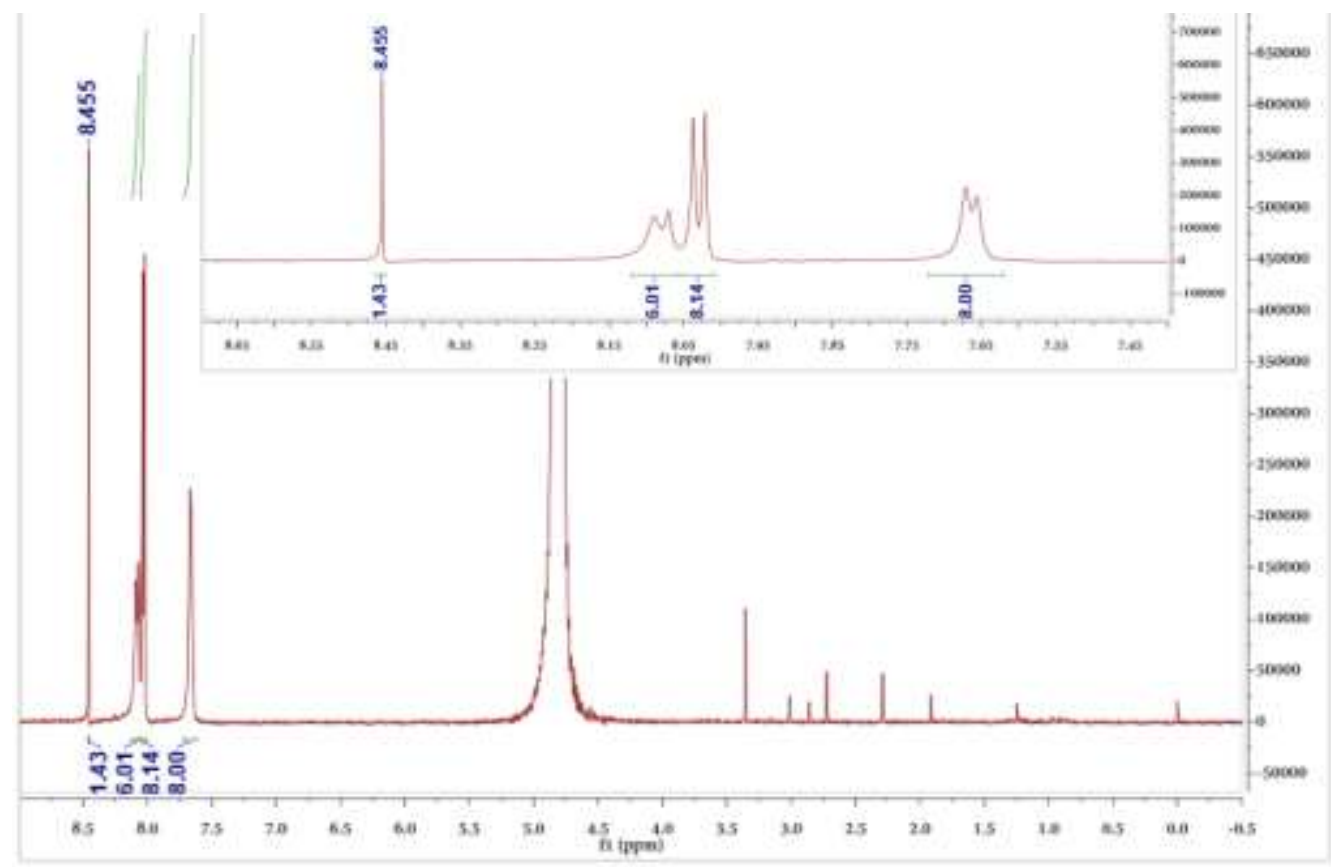

Figure S31. ${ }^{1} \mathrm{H}$ NMR spectrum of R1-NU-1000-F. The sample was digested in $0.1 \mathrm{M} \mathrm{NaOD} / \mathrm{D}_{2} \mathrm{O}$ and diluted in $\mathrm{D}_{2} \mathrm{O}$. Formate with the amount of 2.86 per $\mathrm{Zr}_{6}$ node returned to the node after heating NU1000-FF-Cl at $100^{\circ} \mathrm{C}$ in a solution of $\mathrm{DMF} / \mathrm{HCl}$ overnight. 


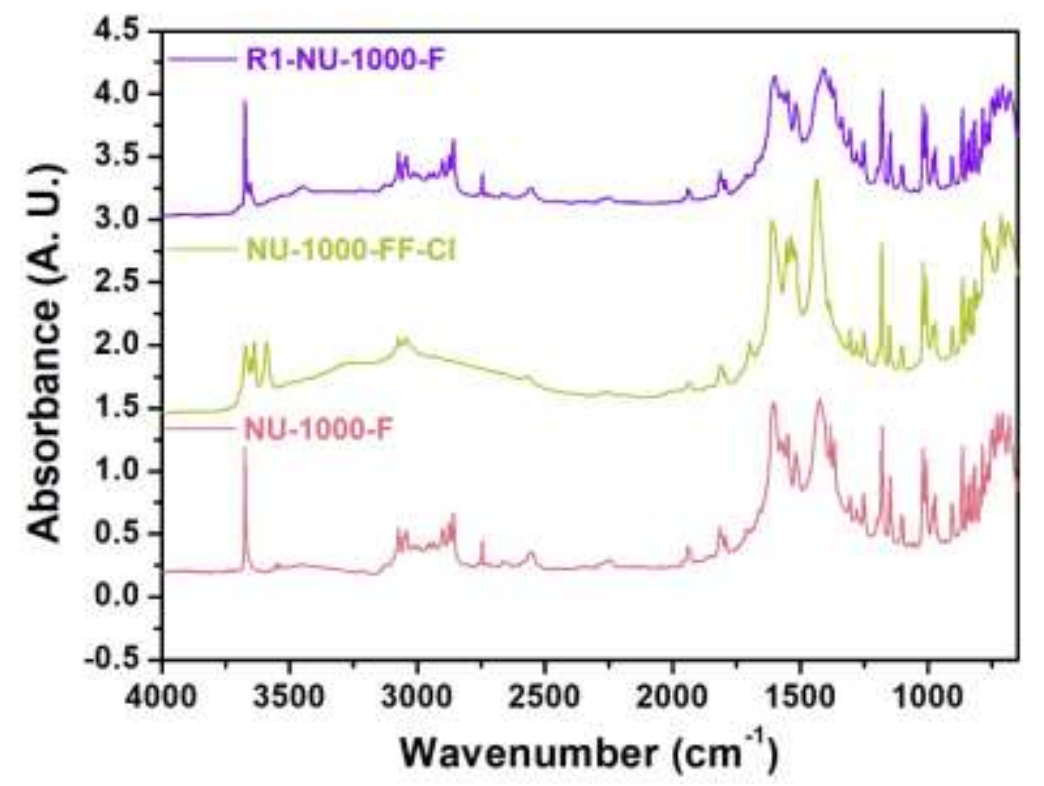

Figure S32. DRIFTS spectra of NU-1000-F, NU-1000-FF-CI, and R1-NU-1000-F. As a characteristic $\mathrm{C}-\mathrm{H}$ vibration of the formate ligand, the band at $2745 \mathrm{~cm}^{-1}$ returns after heating FF-NU-1000 at $100{ }^{\circ} \mathrm{C}$ in a solution of $\mathrm{DMF} / \mathrm{HCl}$ overnight.
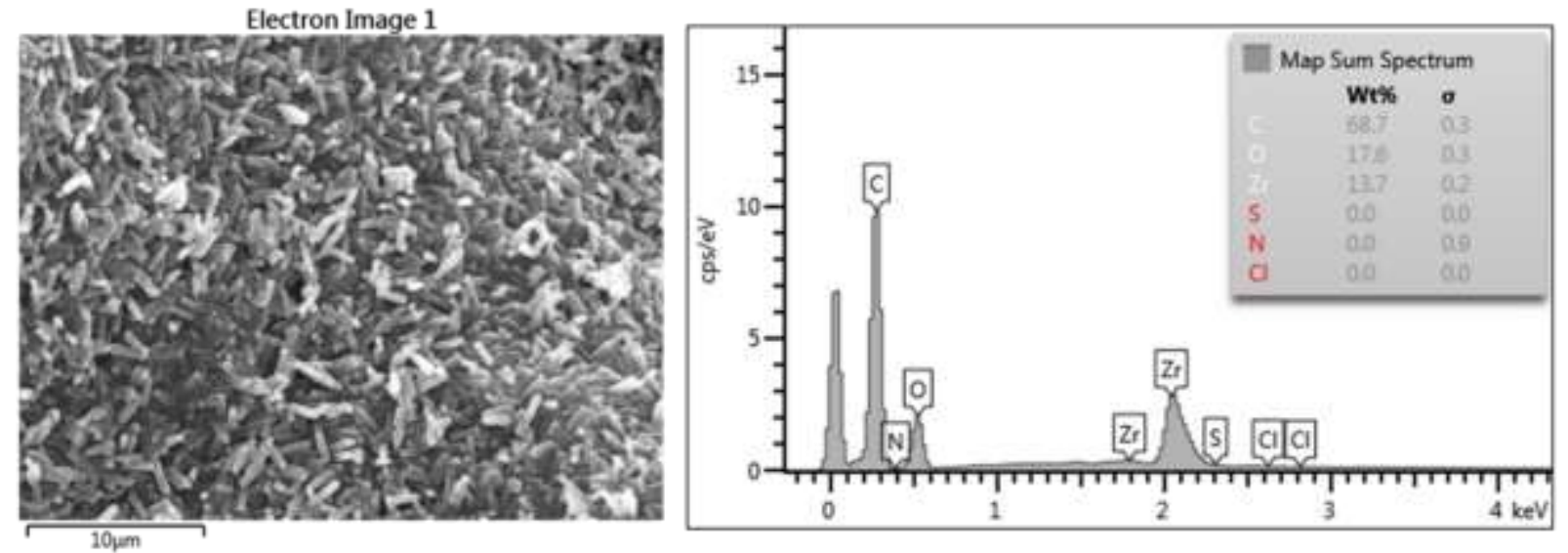

Figure S33. SEM image and EDS elemental mapping analysis of R1-NU-1000-F regenerated from NU1000-FF-Cl. Notice that no $\mathrm{Cl}$ is detectable after heating NU-1000-FF-Cl overnight at $373 \mathrm{~K}$ in a solution of $\mathrm{DMF} / \mathrm{HCl}$. 
a)

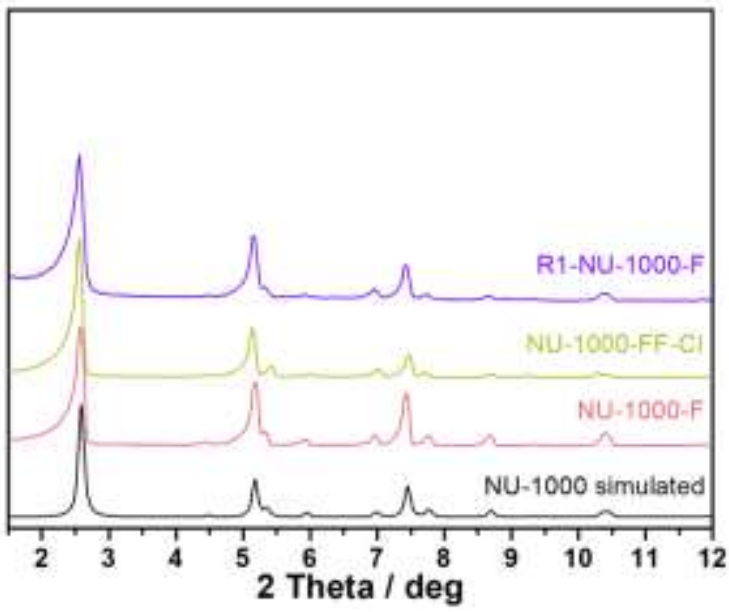

b)

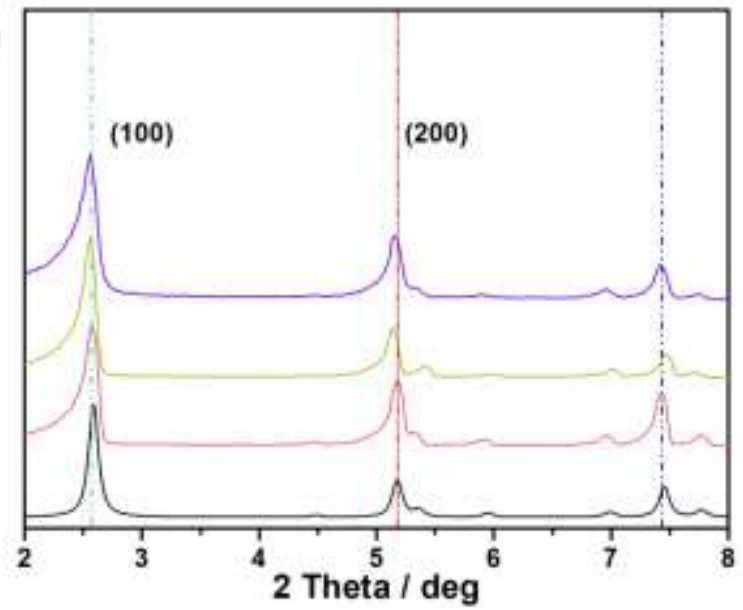

Figure S34. a) PXRD patterns of NU-1000-F (pink), NU-1000-FF-Cl (green), and R1-NU-1000-F generated from NU-1000-FF-Cl (violet); b) amplified version of a) showing the range between $2^{\circ}$ to $8^{\circ}$.

\section{Access to NU-1000-FF by Base-washing}
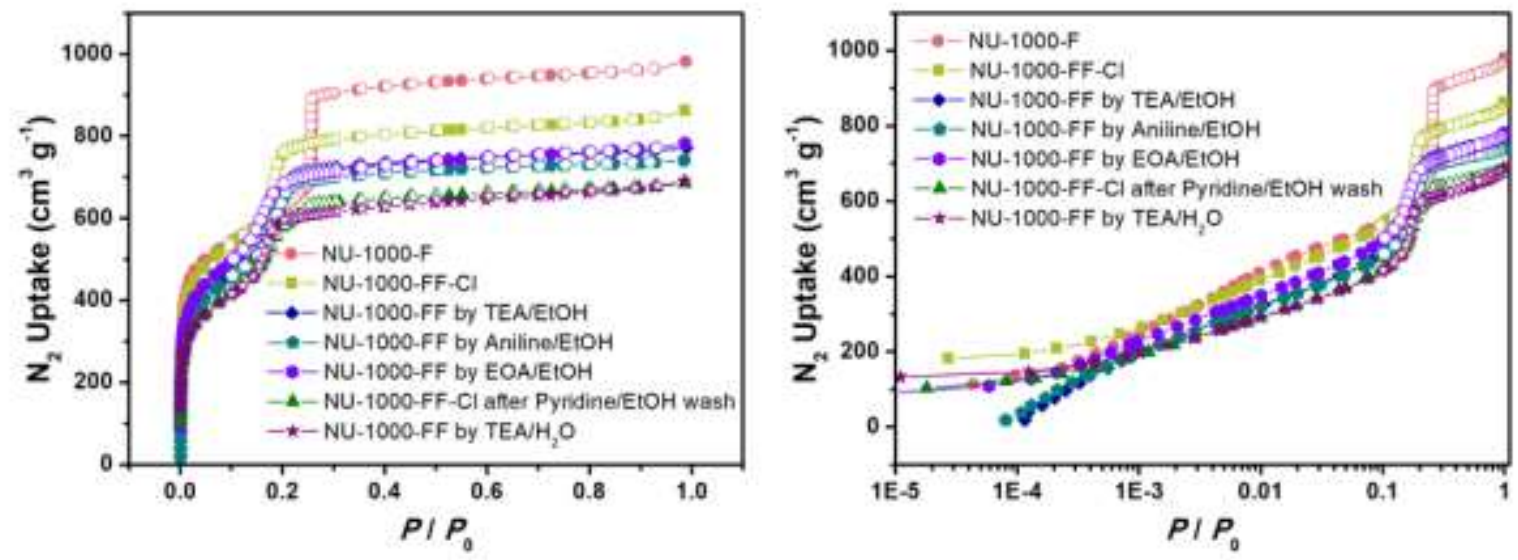

Figure S35. $\mathrm{N}_{2}$ adsorption isotherms at $77 \mathrm{~K}$ for NU-1000-F (red-pink circle), for NU-1000-FF-Cl (yellow-green square), and for NU-1000-FF obtained via treatment of NU-1000-FF-Cl with the following weak bases: TEA (blue diamond) in ethanol, aniline (blue-green pentagon) in ethanol, EOA (violet hexagon), pyridine (green triangle) in ethanol, and TEA (purple star) in $\mathrm{H}_{2} \mathrm{O}$. Left: linear $\mathrm{P} / \mathrm{P}_{0}$ scale; right: logarithmic $\mathrm{P} / \mathrm{P}_{0}$ scale. 


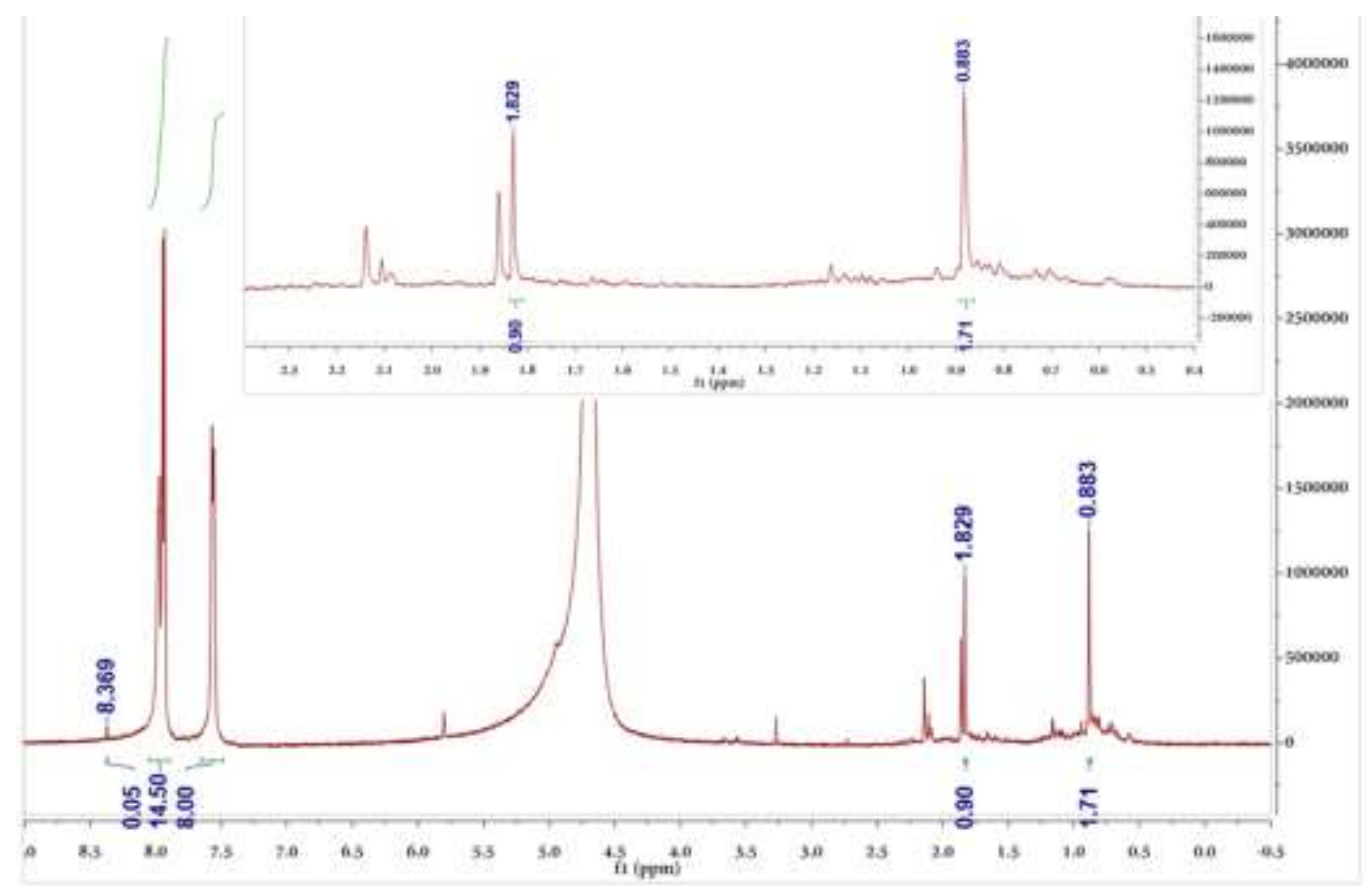

Figure S36. ${ }^{1} \mathrm{H}$ NMR spectrum of NU-1000-FF by washing NU-1000-FF-Cl with TEA in ethanol. The sample was digested in $0.1 \mathrm{M} \mathrm{NaOD} / \mathrm{D}_{2} \mathrm{O}$ and diluted in $\mathrm{D}_{2} \mathrm{O}$.

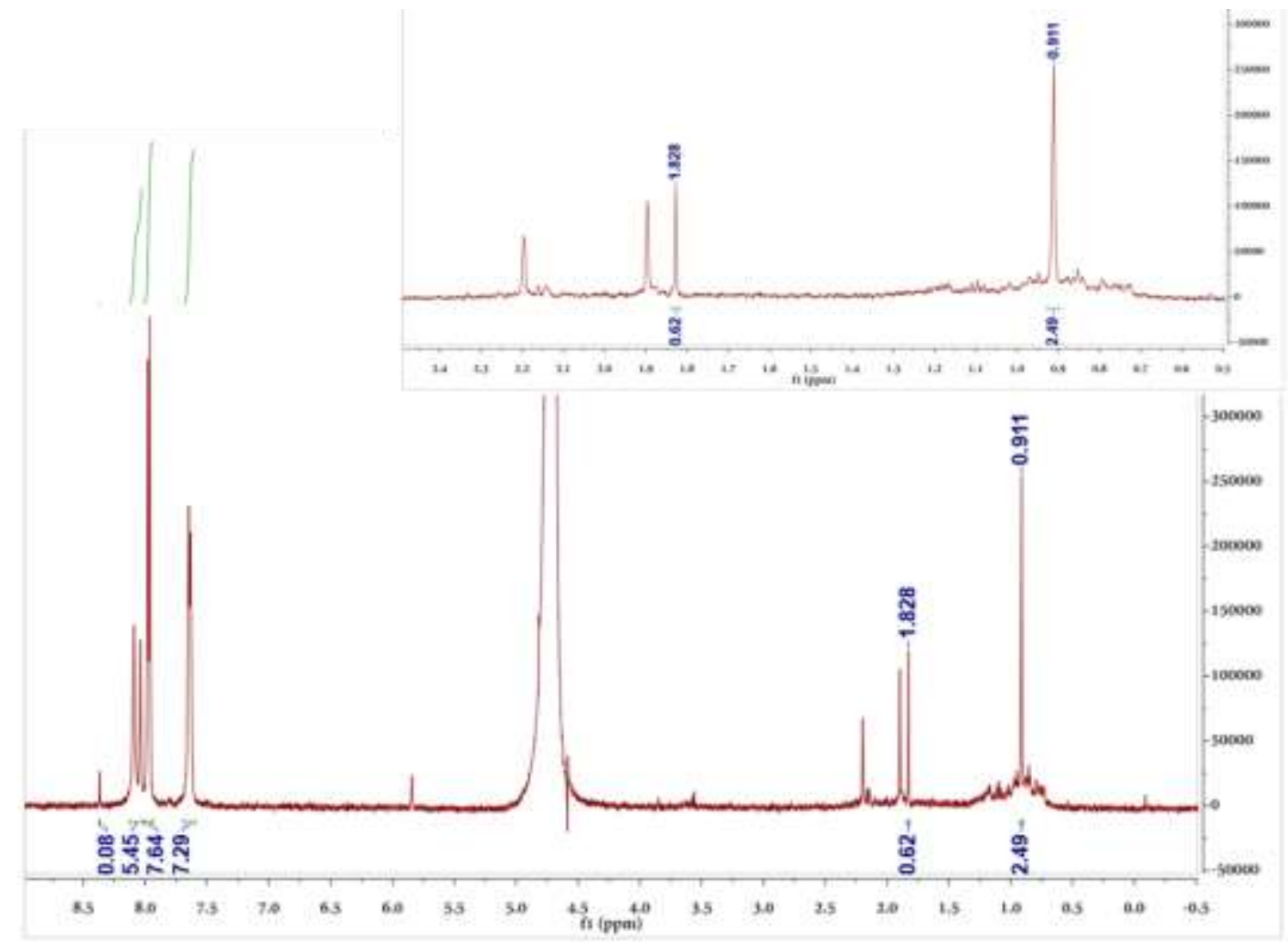

Figure S37. ${ }^{1} \mathrm{H}$ NMR spectrum of NU-1000-FF by washing NU-1000-FF-Cl with EOA in ethanol. The sample was digested in $0.1 \mathrm{M} \mathrm{NaOD} / \mathrm{D}_{2} \mathrm{O}$ and diluted in $\mathrm{D}_{2} \mathrm{O}$. 


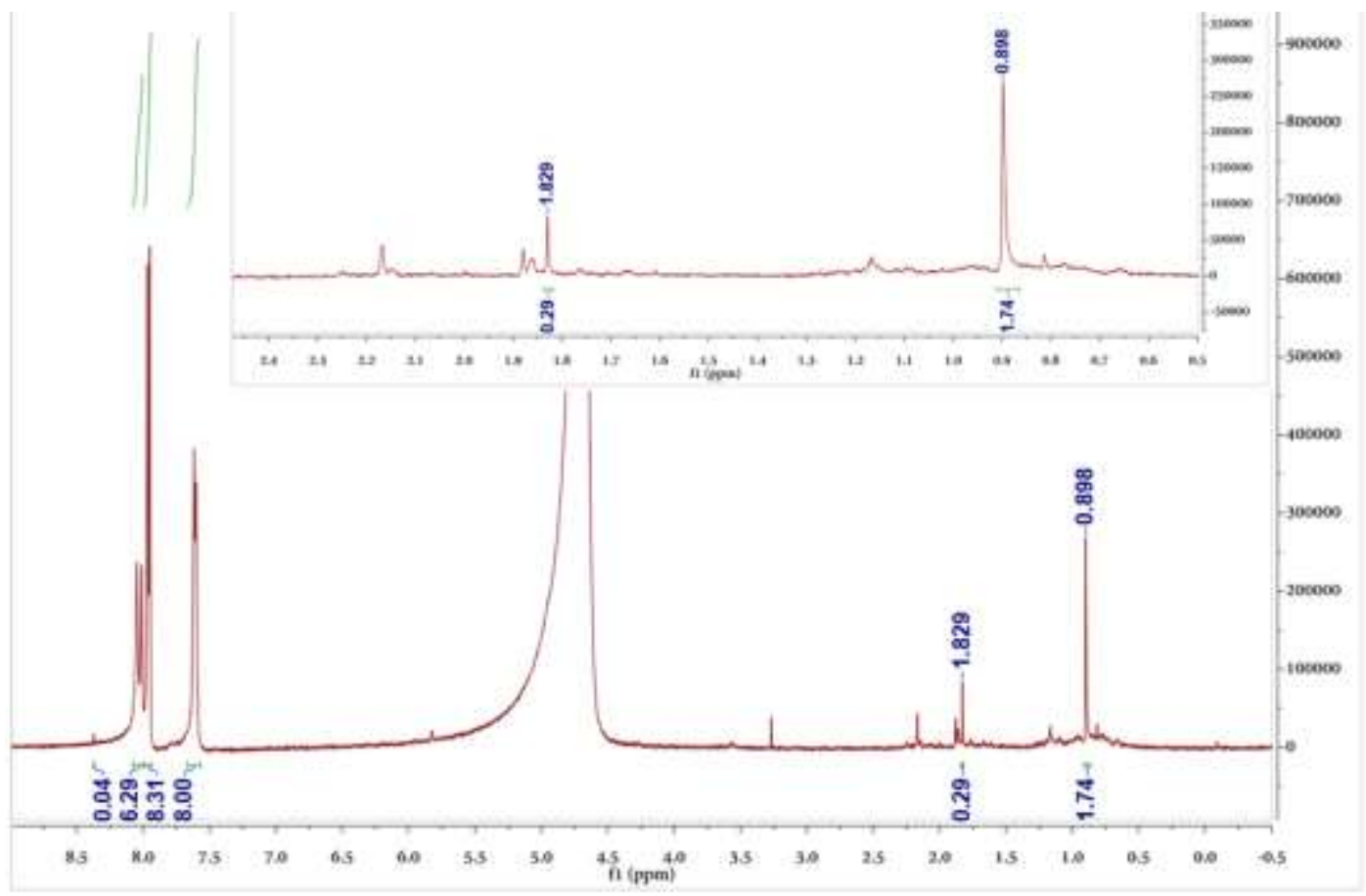

Figure S38. ${ }^{1} \mathrm{H}$ NMR spectrum of NU-1000-FF by washing NU-1000-FF-Cl with TEA in $\mathrm{H}_{2} \mathrm{O}$. The sample was digested in $0.1 \mathrm{M} \mathrm{NaOD} / \mathrm{D}_{2} \mathrm{O}$ and diluted in $\mathrm{D}_{2} \mathrm{O}$.

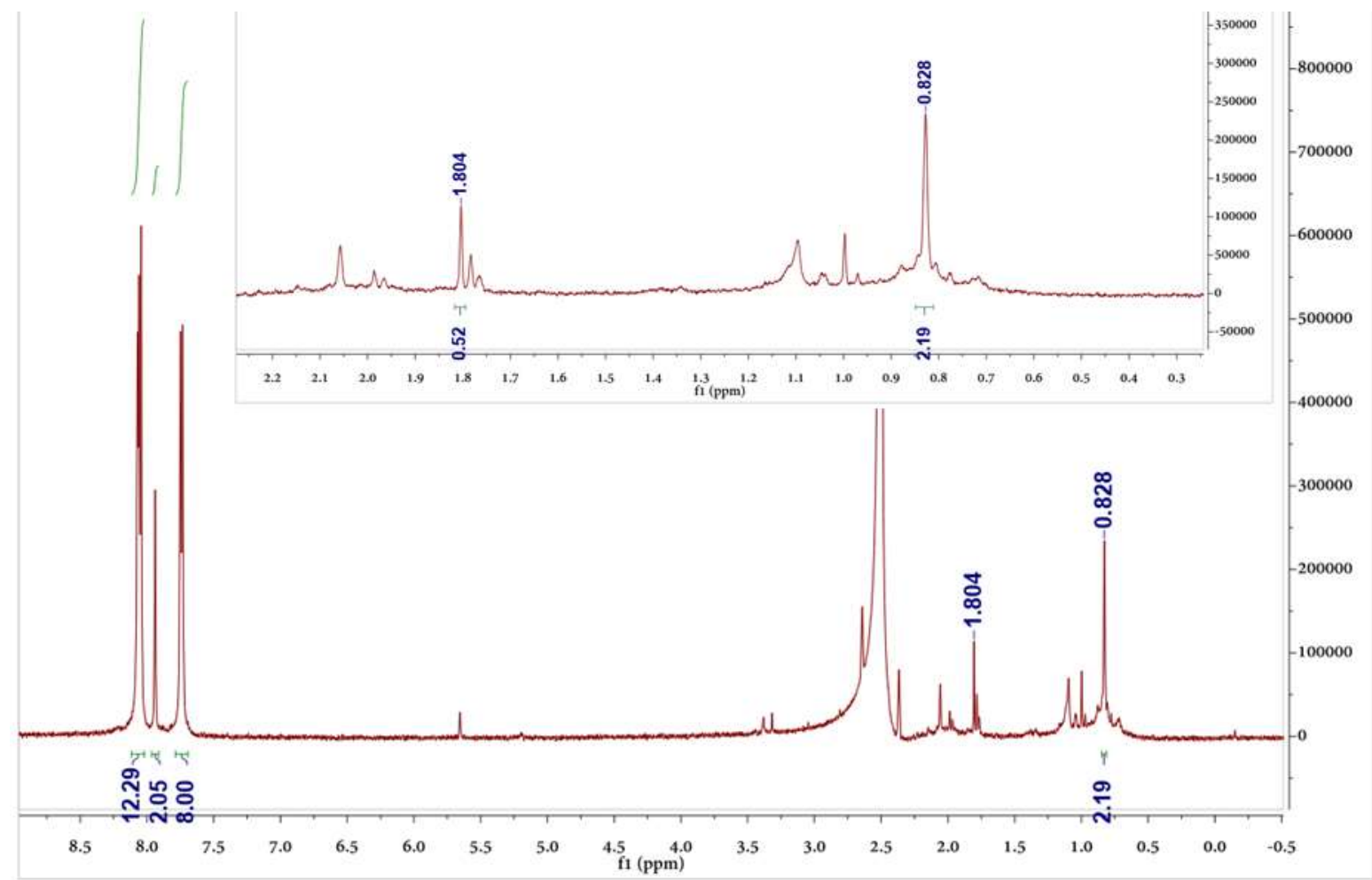

Figure S39. ${ }^{1} \mathrm{H}$ NMR spectrum of NU-1000-FF by washing NU-1000-FF-Cl with TEA in $\mathrm{H}_{2} \mathrm{O}$. The sample was digested in $\mathrm{D}_{2} \mathrm{SO}_{4}$ and diluted in $d_{6}$-DMSO. 

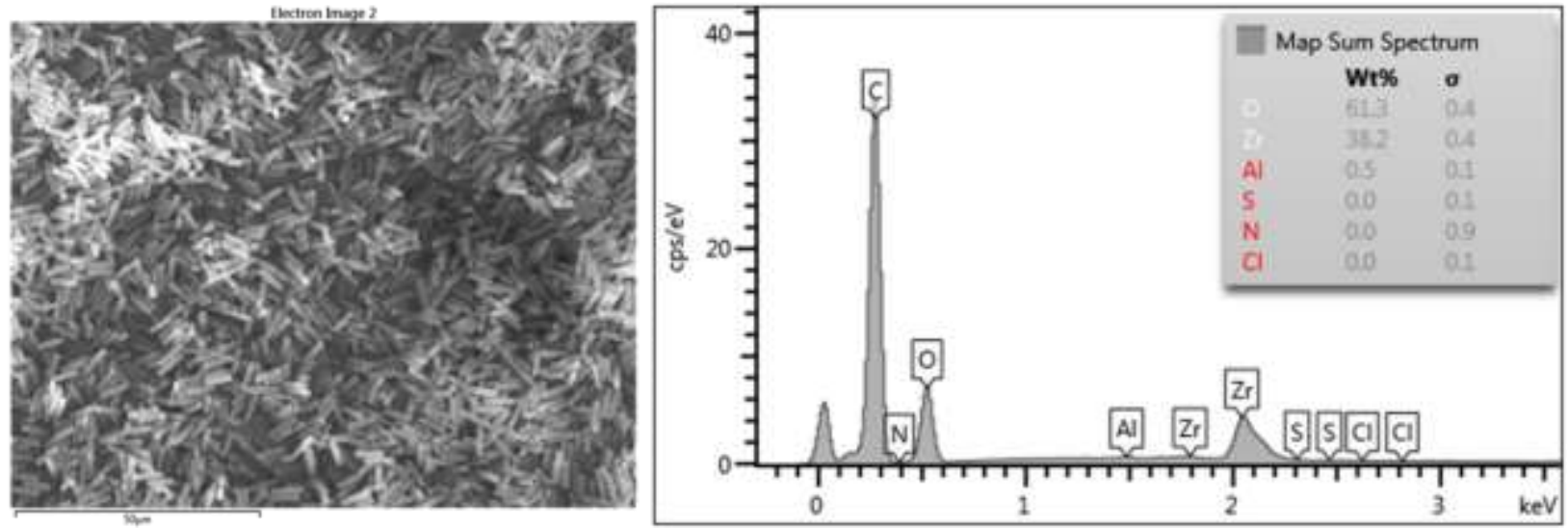

Figure S40. SEM image and EDS elemental mapping analysis of NU-1000-FF by washing NU-1000FF-Cl with TEA in ethanol. It clearly reveals that no $\mathrm{Cl}$ is left after washing.
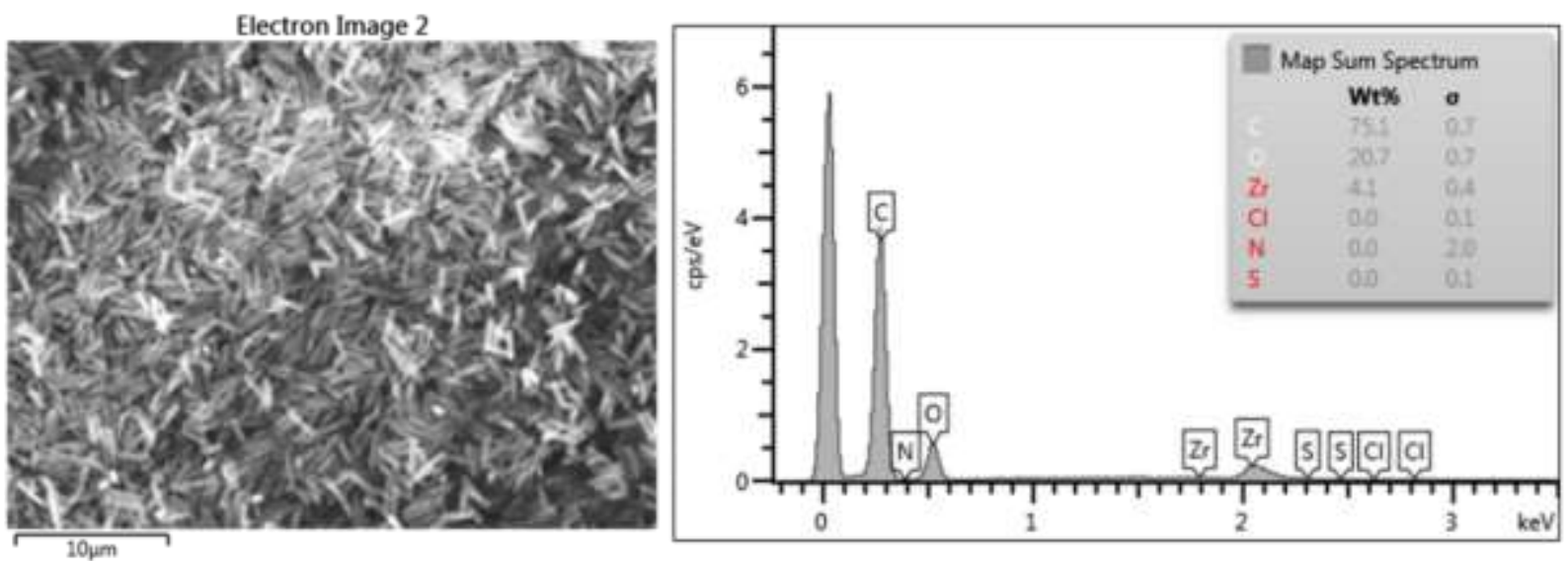

Figure S41. SEM image and EDS elemental mapping analysis of NU-1000-FF by washing NU-1000FF-Cl with EOA in ethanol. It clearly reveals that no $\mathrm{Cl}$ is left after washing.
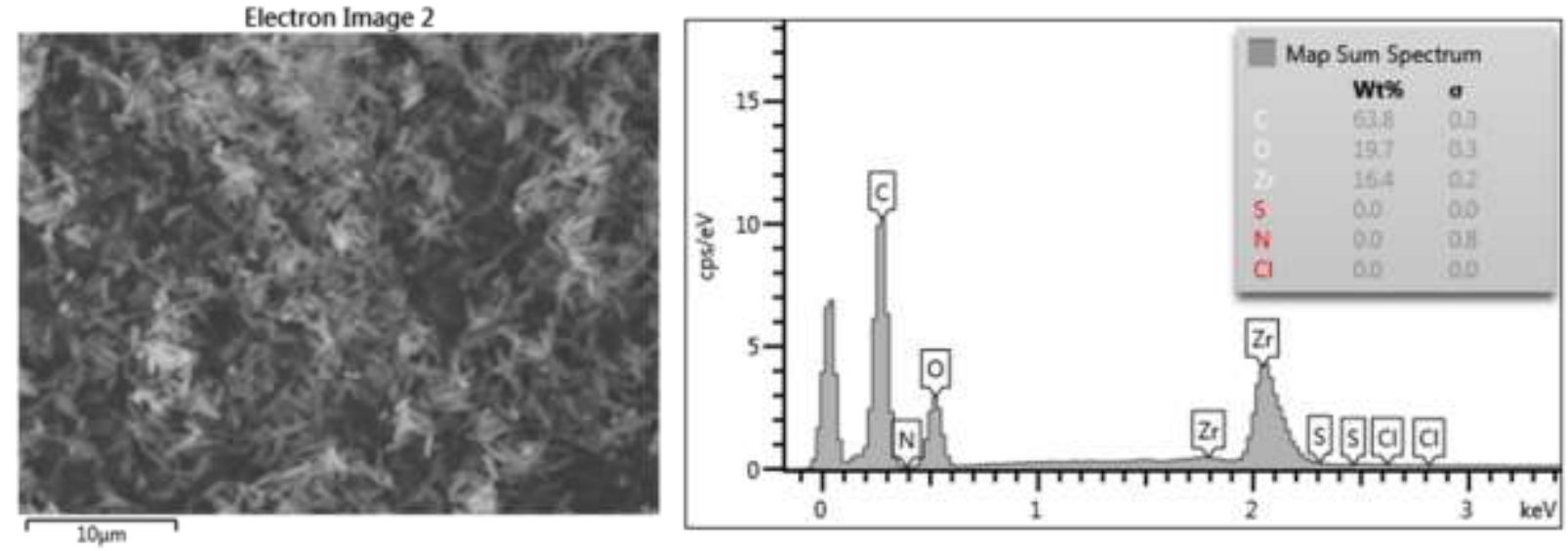

Figure S42. SEM image and EDS elemental mapping analysis of NU-1000-FF by washing NU-1000FF-Cl with TEA in $\mathrm{H}_{2} \mathrm{O}$. It clearly reveals that no $\mathrm{Cl}$ is left after washing. 

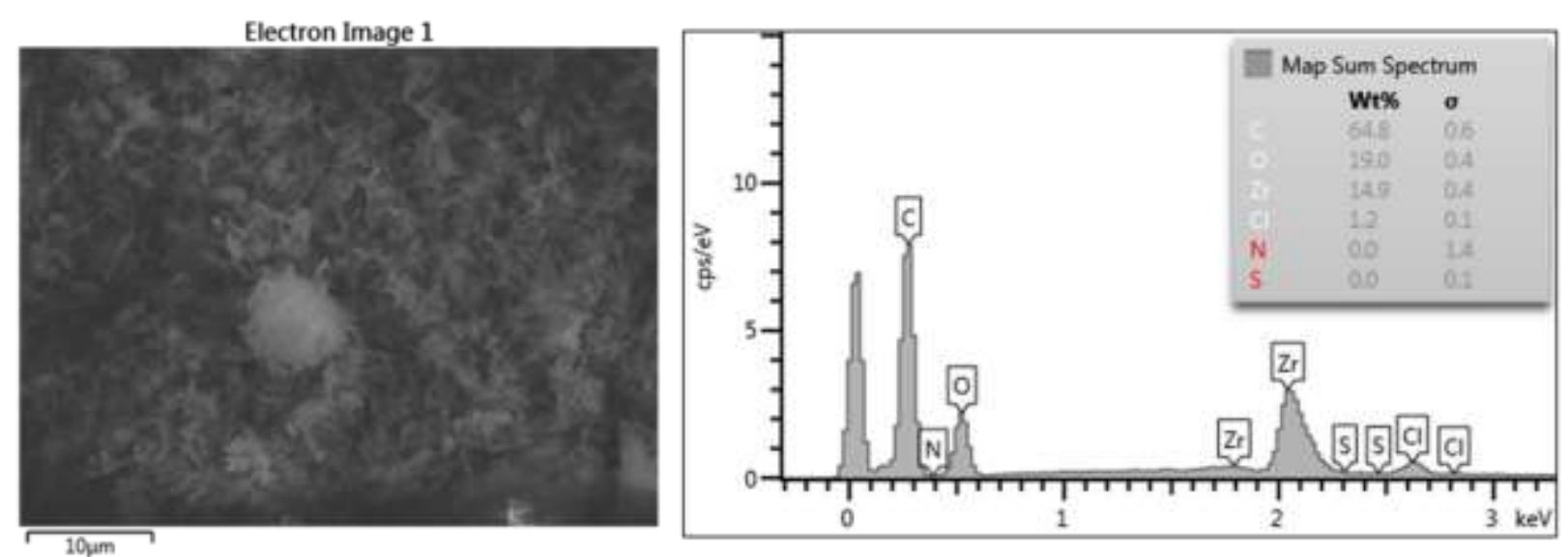

Figure S43. SEM image and EDS elemental mapping analysis of FF-NU-1000 washed twice with pyridine in ethanol. There remain about $1.2 \mathrm{Cl}$ atoms per $\mathrm{Zr}_{6}$ node.

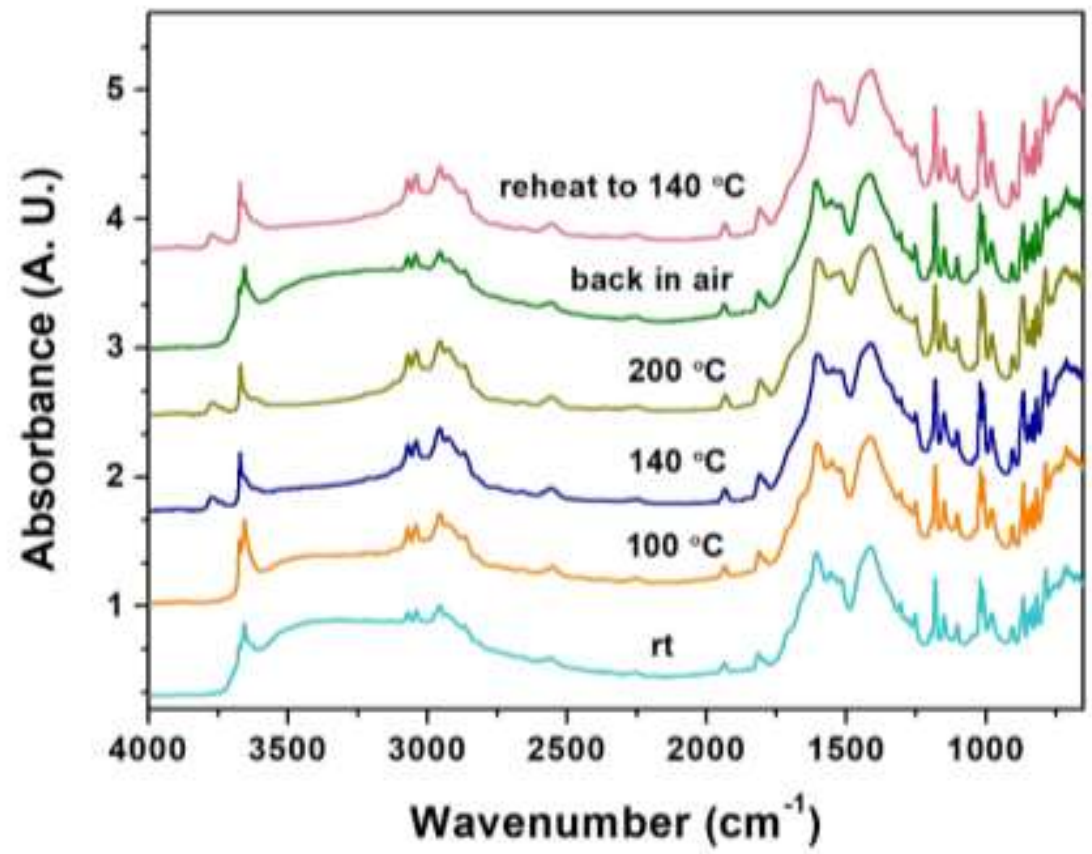

Figure S44. DRIFTS spectra of NU-1000-FF at various temperatures. 
a)

b)

c)

Figure S45. Photos of NU-1000-F (a, pale yellow), NU-1000-FF-Cl (b, yellow), and NU-1000-FF (c, golden yellow).

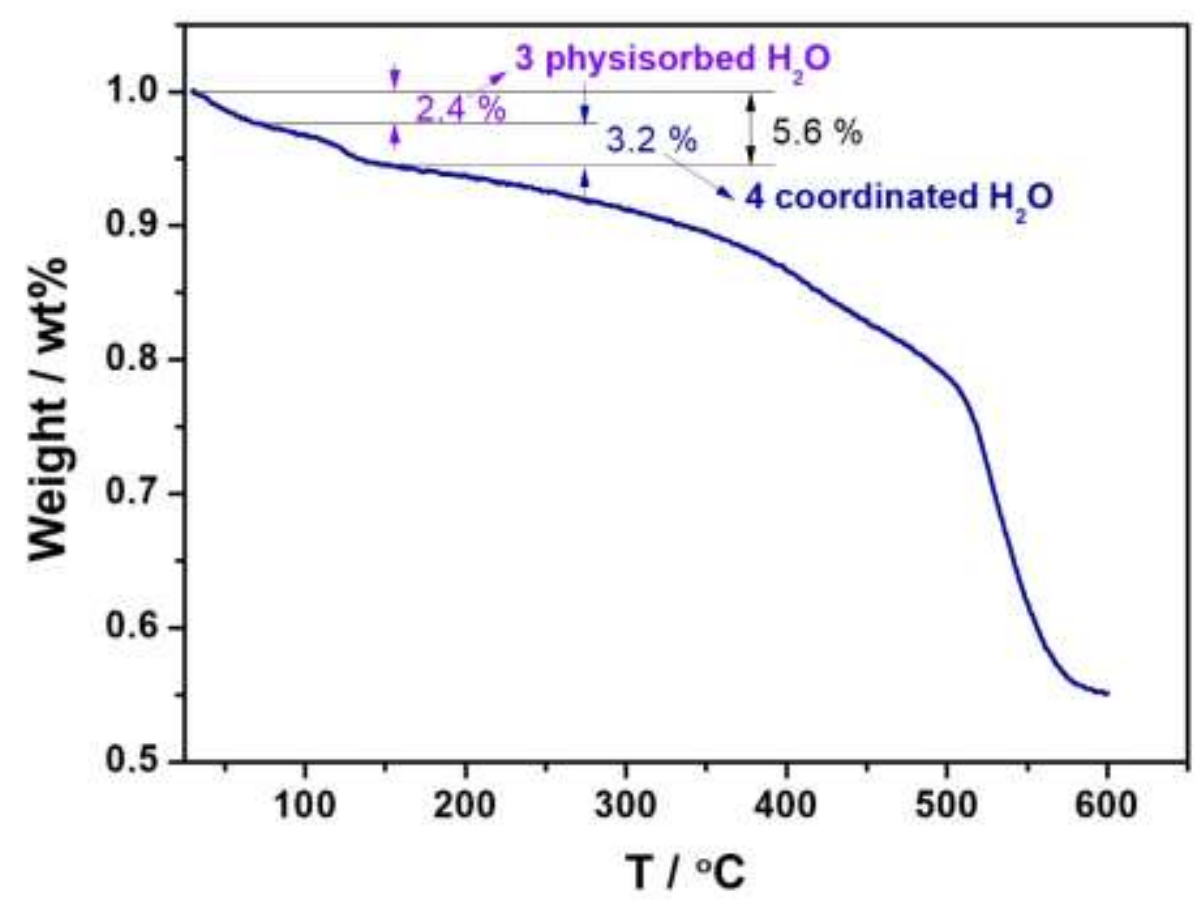

Figure S46. TG curve of NU-1000-FF indicating a loss of 4 coordinated $\mathrm{H}_{2} \mathrm{O}$ at $80 \sim 150{ }^{\circ} \mathrm{C}$. 


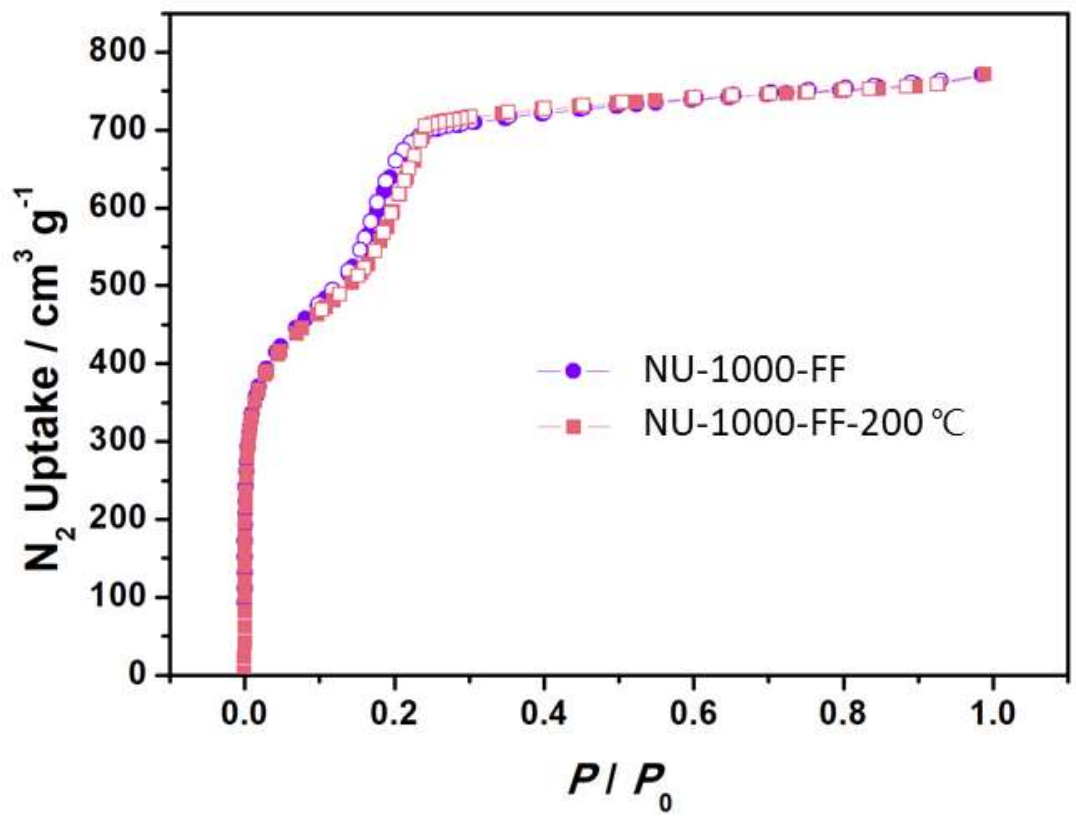

Figure S47. $\mathrm{N}_{2}$ adsorption isotherms at $77 \mathrm{~K}$ for NU-1000-FF activated at $120^{\circ} \mathrm{C}$ overnight (violet circle) and NU-1000-FF activated at $200{ }^{\circ} \mathrm{C}$ overnight.

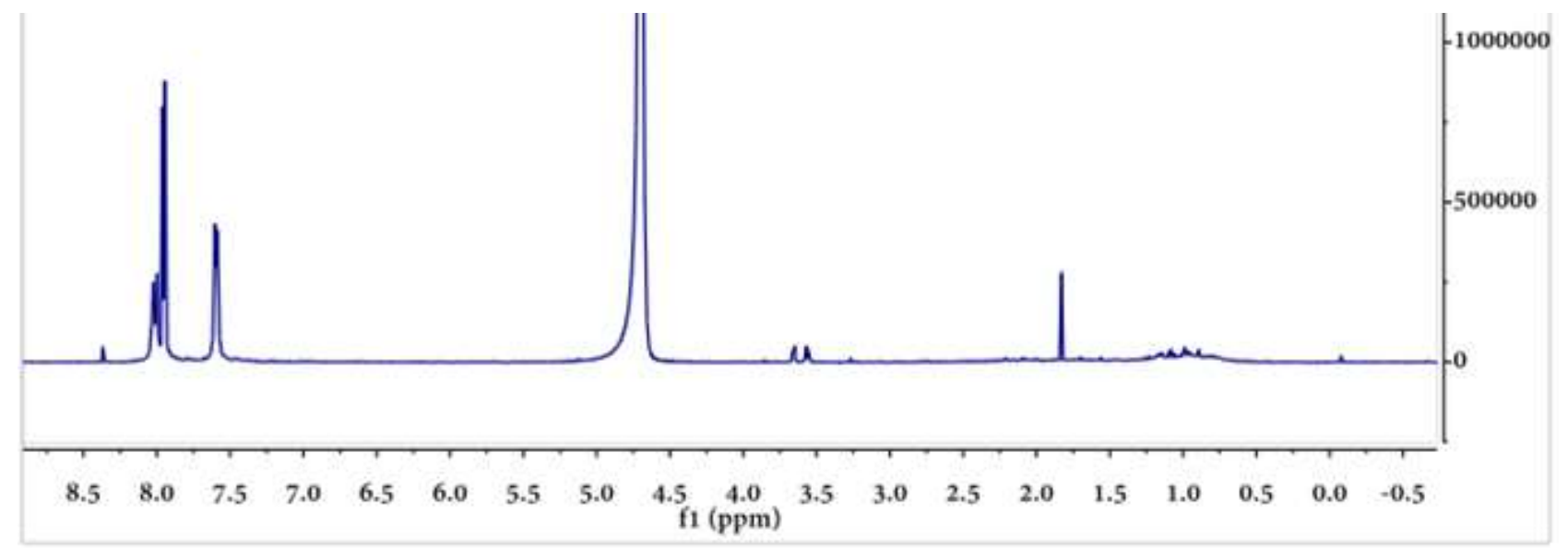

Figure S48. ${ }^{1} \mathrm{H}$ NMR spectrum of NU-1000-FF activated at $200{ }^{\circ} \mathrm{C}$ overnight. The sample was digested in $0.1 \mathrm{M} \mathrm{NaOD} / \mathrm{D}_{2} \mathrm{O}$ and diluted in $\mathrm{D}_{2} \mathrm{O}$. The peak at around $0.883 \mathrm{ppm}$ disappeared. 


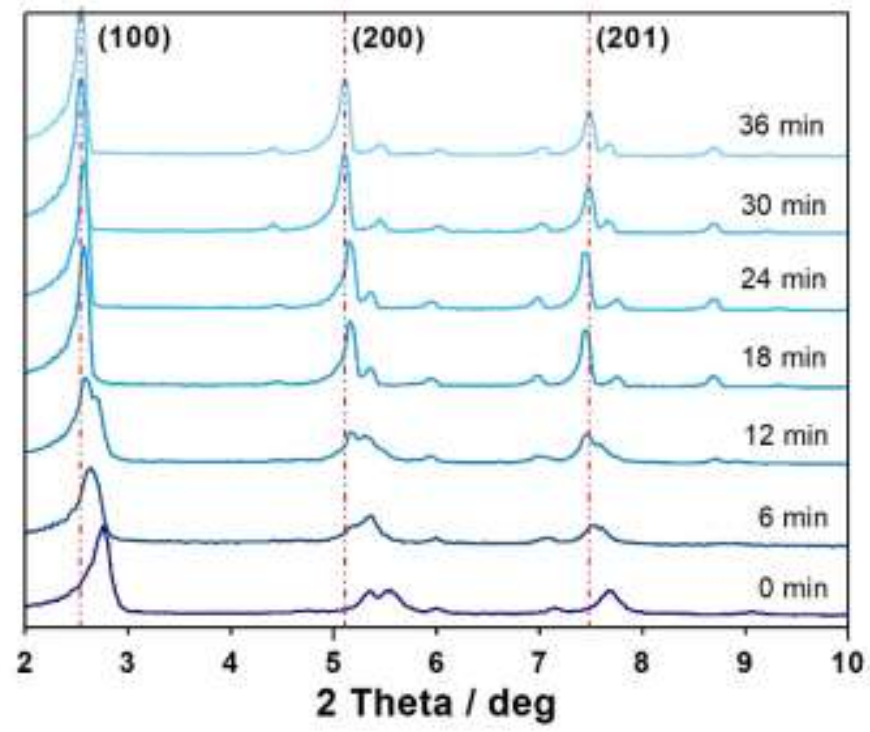

Figure S49. PXRD patterns of NU-1000-FF indicating a gradually change from the dehydrated version to the hydrated version. NU-1000-FF were packed into PXRD mask in glovebox with the protection of film covered. The PXRD patterns were scanned repeatedly without an interval, and each scan from $1^{\circ}$ to $30^{\circ}$ costed about $6 \mathrm{~min}$.

\section{NU-1000-FF Changing Back to NU-1000-F (denoted as R2-NU-1000-F)}
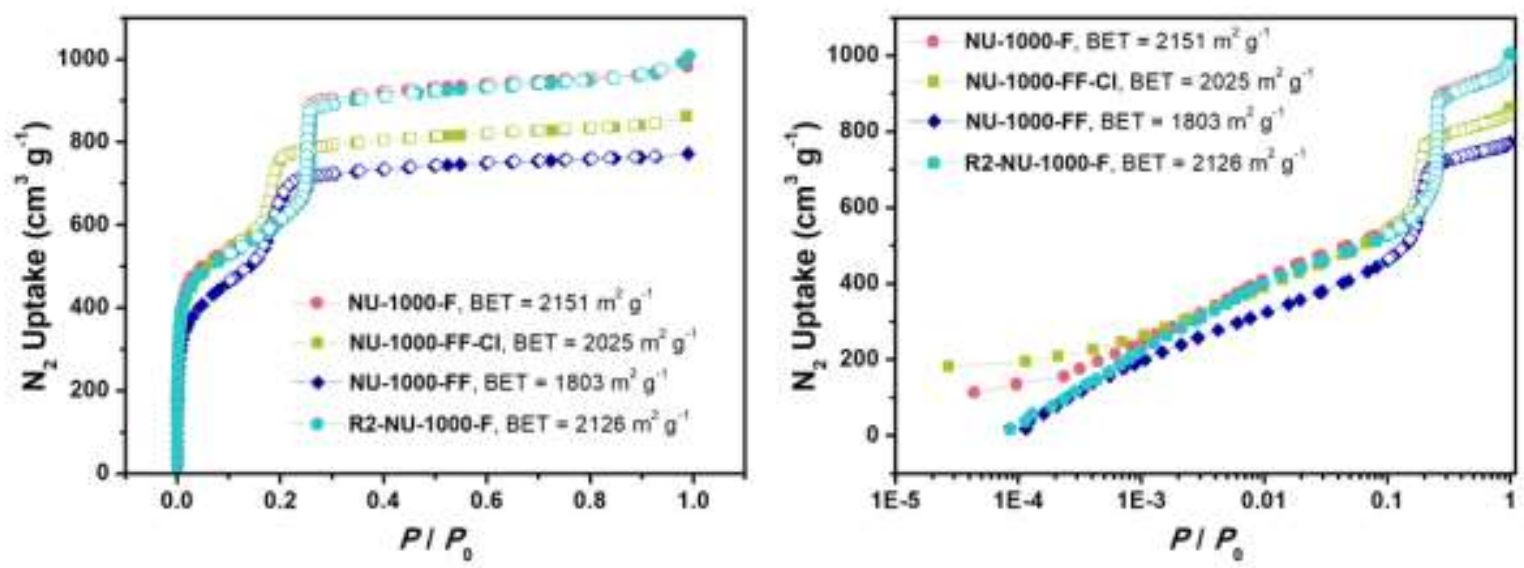

Figure S50. $\mathrm{N}_{2}$ adsorption isotherms at $77 \mathrm{~K}$ for NU-1000-F (pink circle), and NU-1000-FF-Cl (green square), NU-1000-FF (navy diamond), and R2-NU-1000-F regenerated from NU-1000-FF (blue pentagon). Left: linear $\mathrm{P} / \mathrm{P}_{0}$ scale; right: logarithmic $\mathrm{P} / \mathrm{P}_{0}$ scale. 


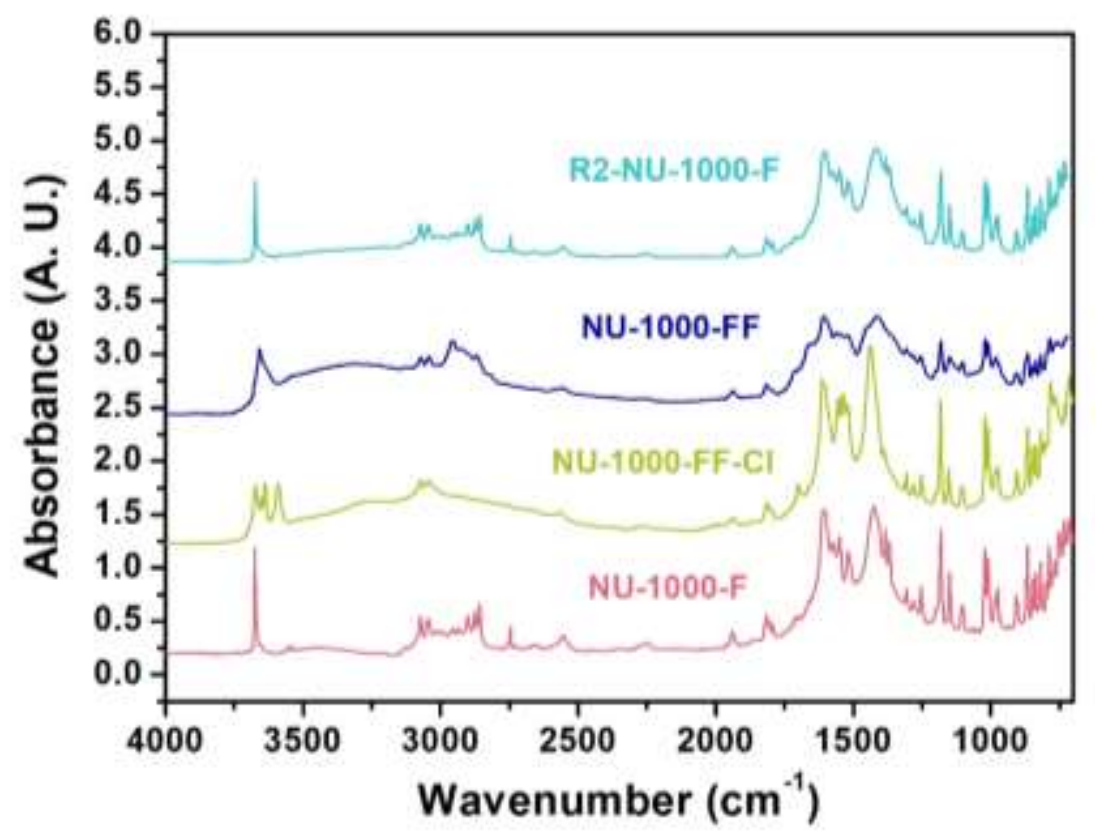

Figure S51. DRIFTS spectra of NU-1000-F, NU-1000-FF-CI, NU-1000-FF, and R2-NU-1000-FF. As a characteristic $\mathrm{C}-\mathrm{H}$ vibration of the formate ligand, the band at $2745 \mathrm{~cm}^{-1}$ returns after heating NU-1000FF overnight at $100^{\circ} \mathrm{C}$ in a solution of $\mathrm{DMF} / \mathrm{HCl}$.
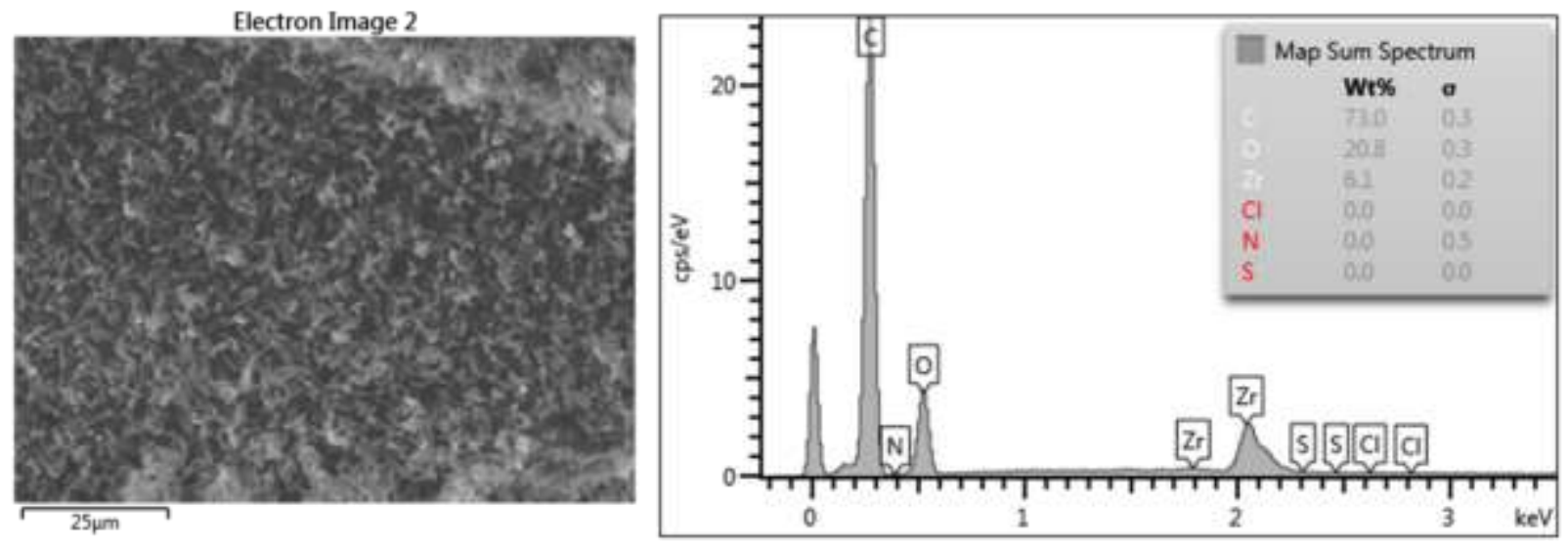

Figure S52. SEM image and EDS elemental mapping analysis of R2-NU-1000-FF. 


\section{Hydrolysis experiments and ${ }^{31}$ P NMR spectra}

Hydrolysis experiments were run at room temperature and the progress of the reaction was monitored by in situ ${ }^{31} \mathrm{P}$ NMR spectroscopy. A solid sample of NU-1000-F, NU-1000-FF or NU-1000-FF-Cl (6 mol\%, $\left.1.5 \mu \mathrm{mol} \mathrm{Zr}_{6}\right)$ was added to $0.4 \mathrm{M}$ N-ethylmorpholine solution $(1.05 \mathrm{~mL} ; 0.05 \mathrm{~mL} \mathrm{~N}$ ethylmorpholine, $0.9 \mathrm{~mL}$ deionized water and $0.1 \mathrm{~mL} \mathrm{D}_{2} \mathrm{O}$ ) in a 1.5 dram vial. The resulting mixture was sonicated for $1 \mathrm{~min}$ to disperse the MOF powder homogeneously. DMNP $(4.0 \mu \mathrm{L}, 25 \mu \mathrm{mol})$ was added to the mixture and swirled for $15 \mathrm{sec}$. The reaction mixture was then transferred to an NMR tube and the spectrum was immediately measured; the first data point was collected $120 \mathrm{sec}$ after the start of the reaction. The progress of the reaction was monitored in $1 \mathrm{~min}$ increments for $1 \mathrm{~h}$ or until $100 \%$ conversion - whichever occurred first (number of scans $=16$, delay time $=28 \mathrm{sec}$ ). Back-ground reactivity was evaluated under identical conditions by in situ ${ }^{31} \mathrm{P}$ NMR, w/o catalyst.

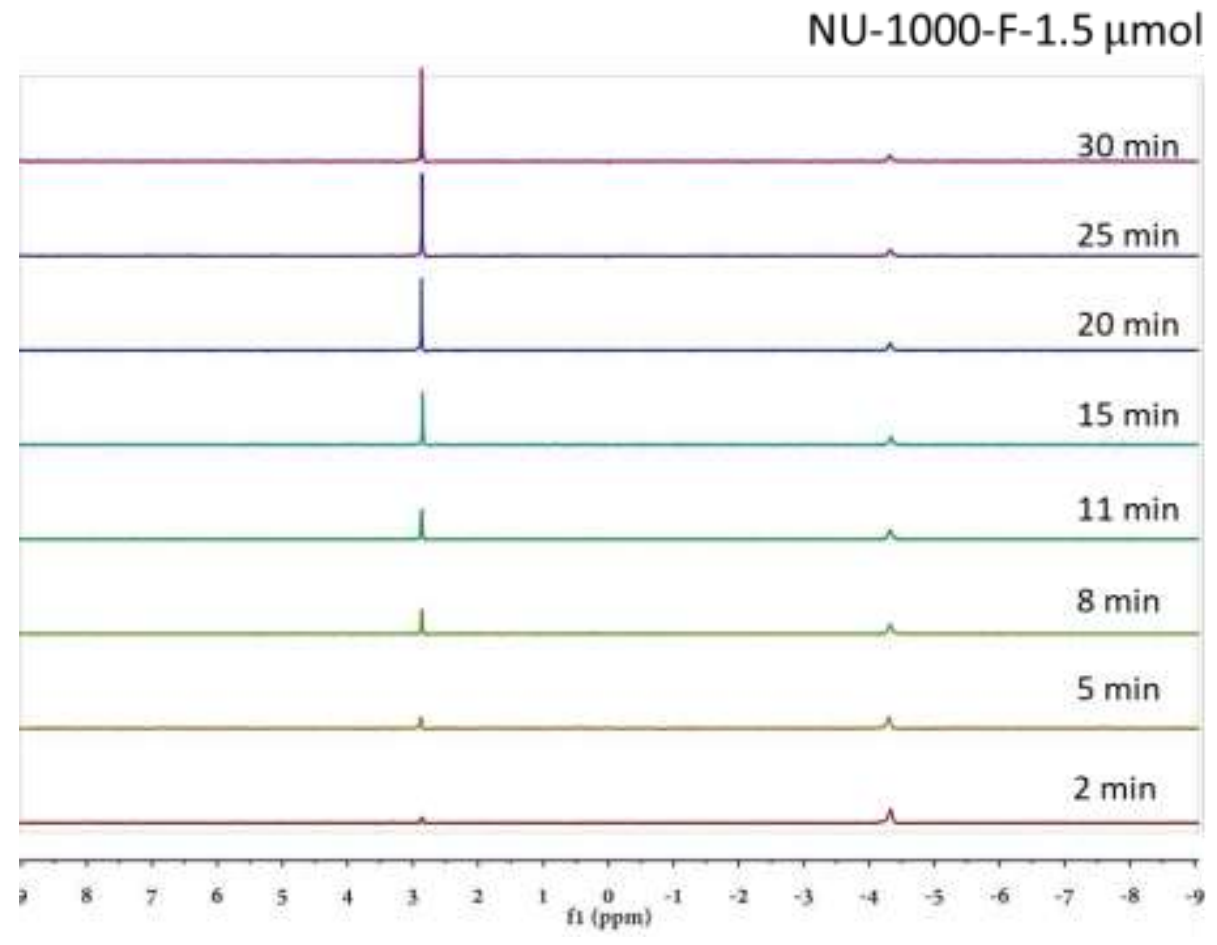

Figure S53. In-situ ${ }^{31} \mathrm{P}$ NMR spectra indicating the progress of hydrolysis of DMNP (-4.3 ppm) to dimethoxy phosphate anion $(2.9 \mathrm{ppm})$ in the presence of $1.5 \mu \mathrm{mol}$ of $\mathbf{N U}-\mathbf{1 0 0 0}-\mathbf{F}$ at room temperature. 
NU-1000-FF-Cl-1.5 $\mu \mathrm{mol}$

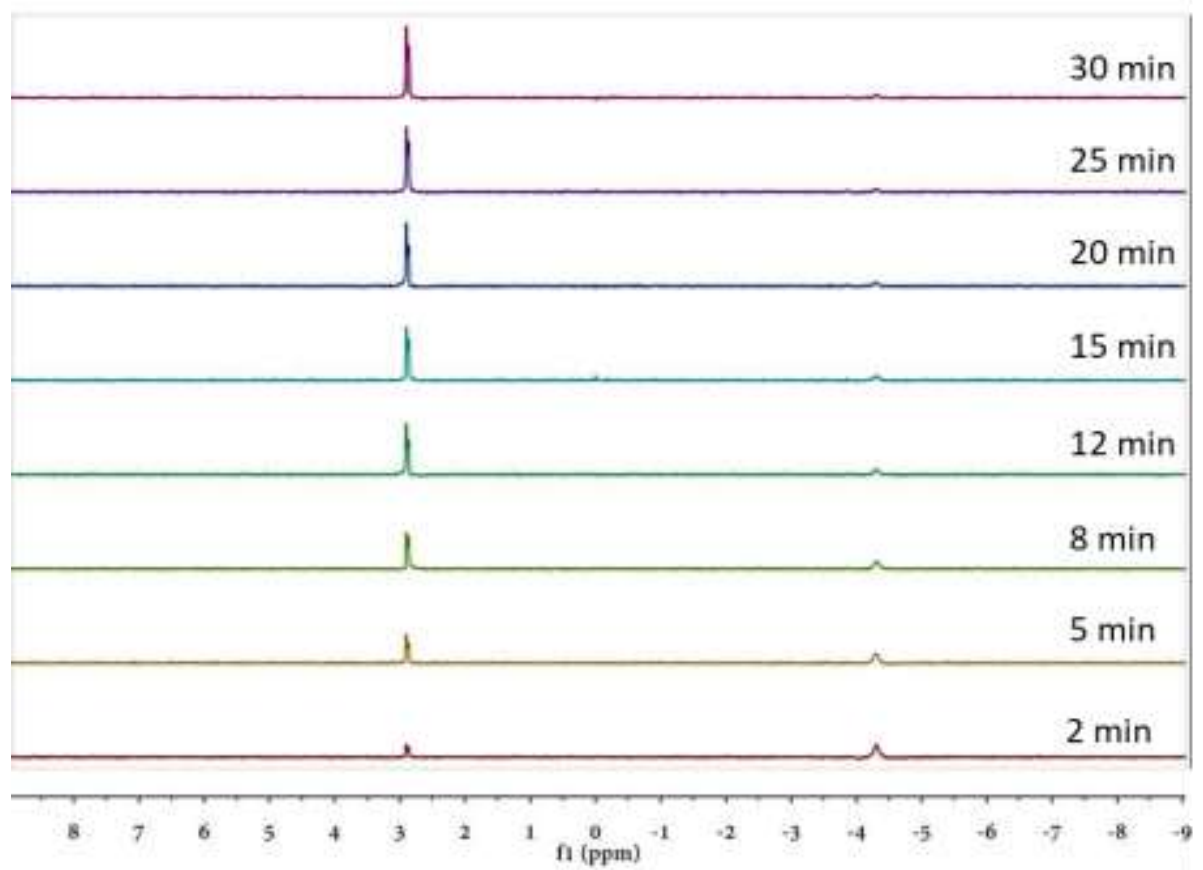

Figure S54. In-situ ${ }^{31} \mathrm{P}$ NMR spectra indicating the progress of hydrolysis of DMNP (-4.3 ppm) to dimethoxy phosphate anion $(2.9 \mathrm{ppm})$ in the presence of $1.5 \mu \mathrm{mol}$ of NU-1000-FF-Cl at room temperature.

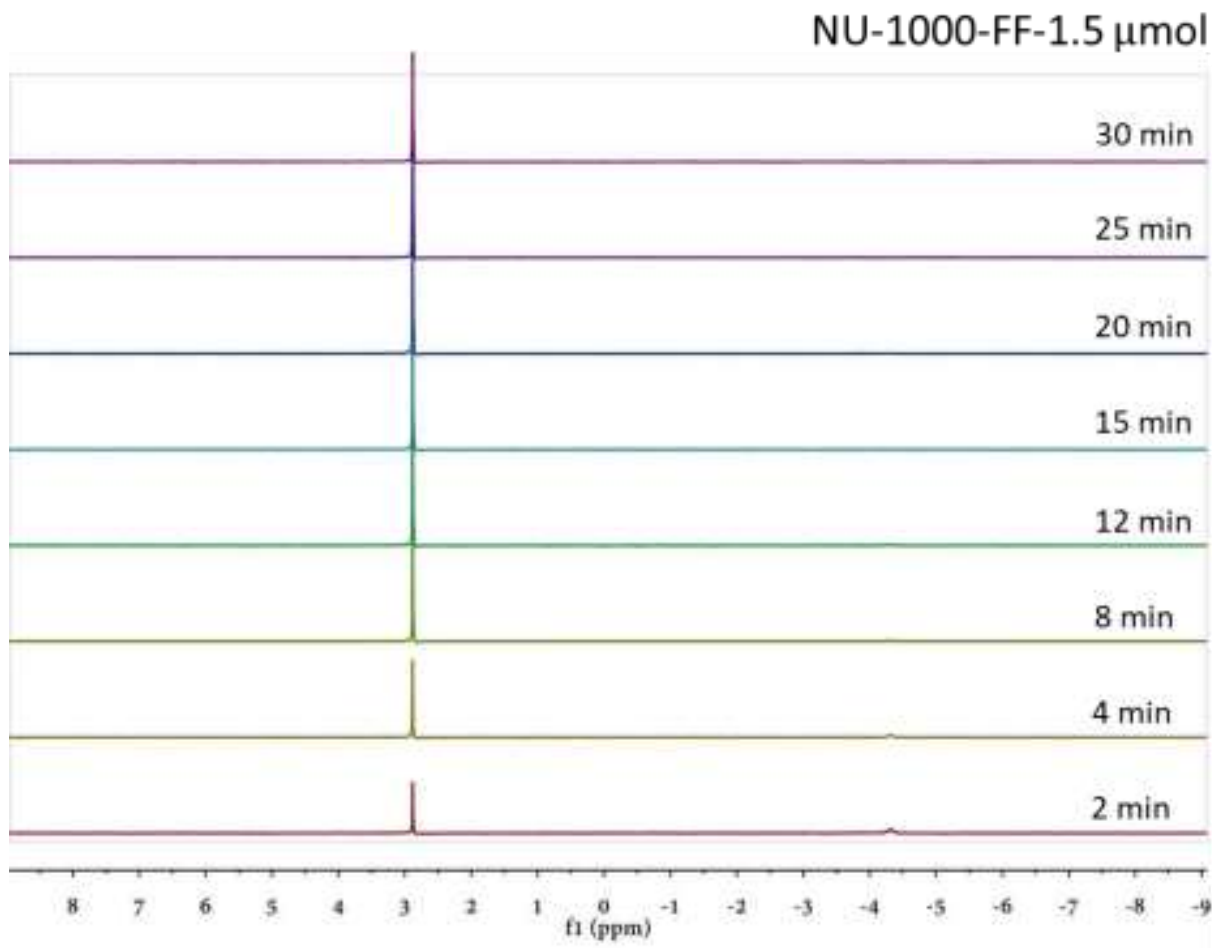

Figure S55. In-situ ${ }^{31} \mathrm{P}$ NMR spectra indicating the progress of hydrolysis of DMNP (-4.3 ppm) to dimethoxy phosphate anion $(2.9 \mathrm{ppm})$ in the presence of $1.5 \mu \mathrm{mol}$ of NU-1000-FF at room temperature. 
NU-1000-FF-0.7 $\mu \mathrm{mol}$

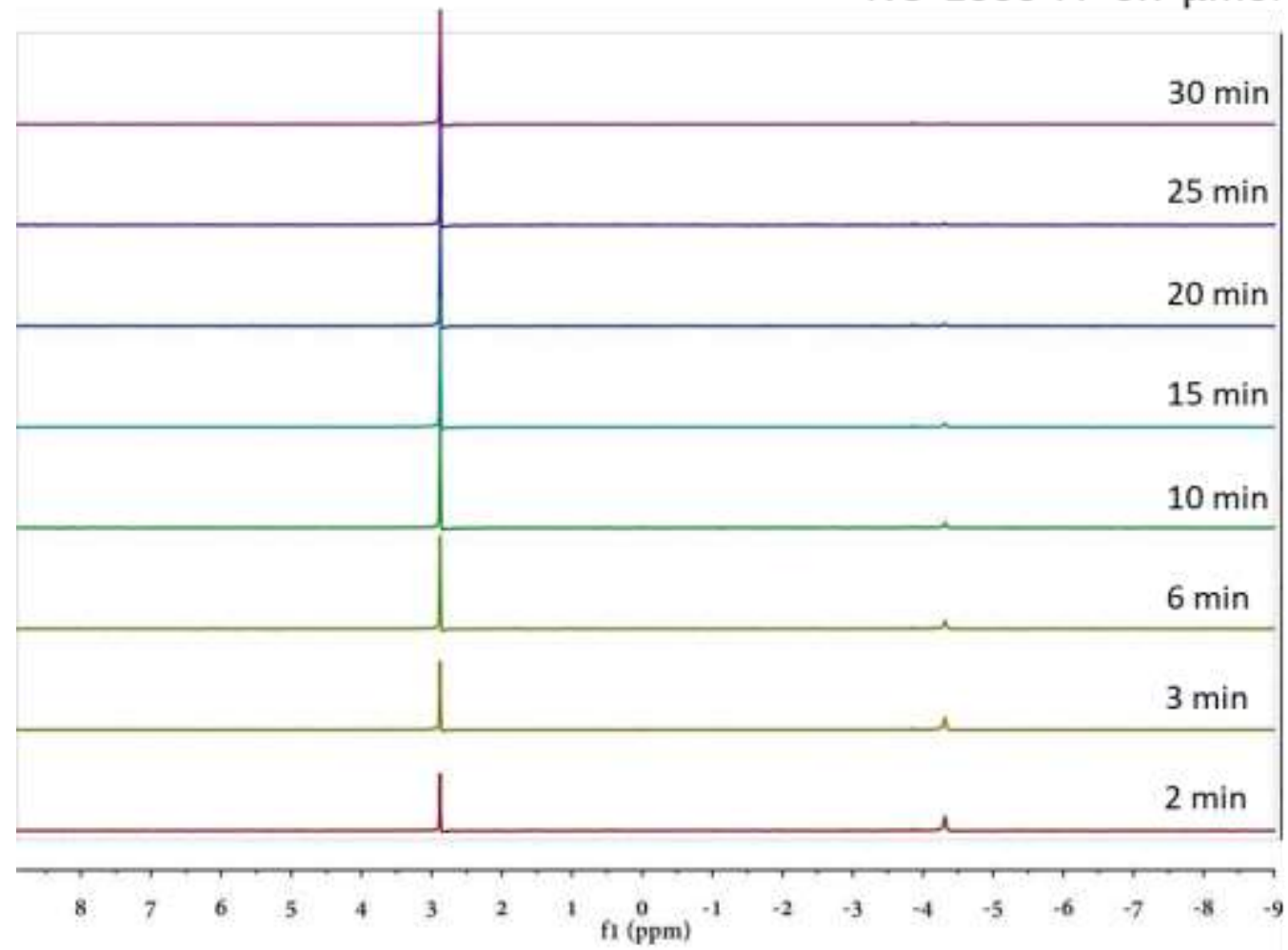

Figure S56. In-situ ${ }^{31} \mathrm{P}$ NMR spectra indicating the progress of hydrolysis of DMNP (-4.3 ppm) to dimethoxy phosphate anion (2.9 ppm) in the presence of $0.7 \mu \mathrm{mol}$ of $\mathbf{N U}-\mathbf{1 0 0 0}-\mathbf{F F}$ at room temperature.

\section{Catalyst Recyclability}

$3.264 \mathrm{mg}$ (6 mol\%, $1.5 \mu \mathrm{mol} \mathrm{Zr} 6) \mathrm{NU}-1000-\mathrm{FF}$ was added to $0.4 \mathrm{M}$ N-ethylmorpholine solution (1.05 mL; $0.05 \mathrm{~mL}$ N-ethylmorpholine, $0.9 \mathrm{~mL}$ deionized water and $0.1 \mathrm{~mL}$ D2O) in a 1.5 dram vial. The resulting mixture was sonicated for $1 \mathrm{~min}$ to disperse the MOF powder uniformly. DMNP (4.0 $\mu \mathrm{L}, 25 \mu \mathrm{mol})$ was added to the mixture and swirled for $10 \mathrm{sec}$. The reaction mixture was then transferred to an NMR tube and the spectrum was immediately measured; for each cycle, the first data point was collected $120 \mathrm{sec}$ after the start of the hydrolysis reaction. The progress of the reaction was monitored in $1 \mathrm{~min}$ increments for $1 \mathrm{~h}$. Between each cycle from the first cycle to the fifth, the catalyst was washed with water six times and soaked in water overnight. It is clear that after the first cycle, the rate of catalysis decreases. For cycles 2 through 5, the rate drops only slightly. One factor is the inevitable physical loss of some amount of catalyst during inter-cycle washing and soaking; unfortunately, given the small absolute amount of catalyst used and the need to account for (or remove) solvent (water) within the porous catalyst, or remove solvent, we did not attempt to quantify the physical losses. We suspect that product inhibition is another. With this in mind, between cycles 5 and 6 we again washed the catalyst six times with water, but then soaked overnight in $0.4 \mathrm{M} \mathrm{N}$ ethylmorpholine in water to facilitate product removal. As shown in the accompanying figure, this 
treatment restores a large fraction of the initial loss in activity. As a result, the hydrolysis speed increased again, and the conversion can reach up to $94 \%$ in $60 \mathrm{~min}$ and $98 \%$ in $90 \mathrm{~min}$.
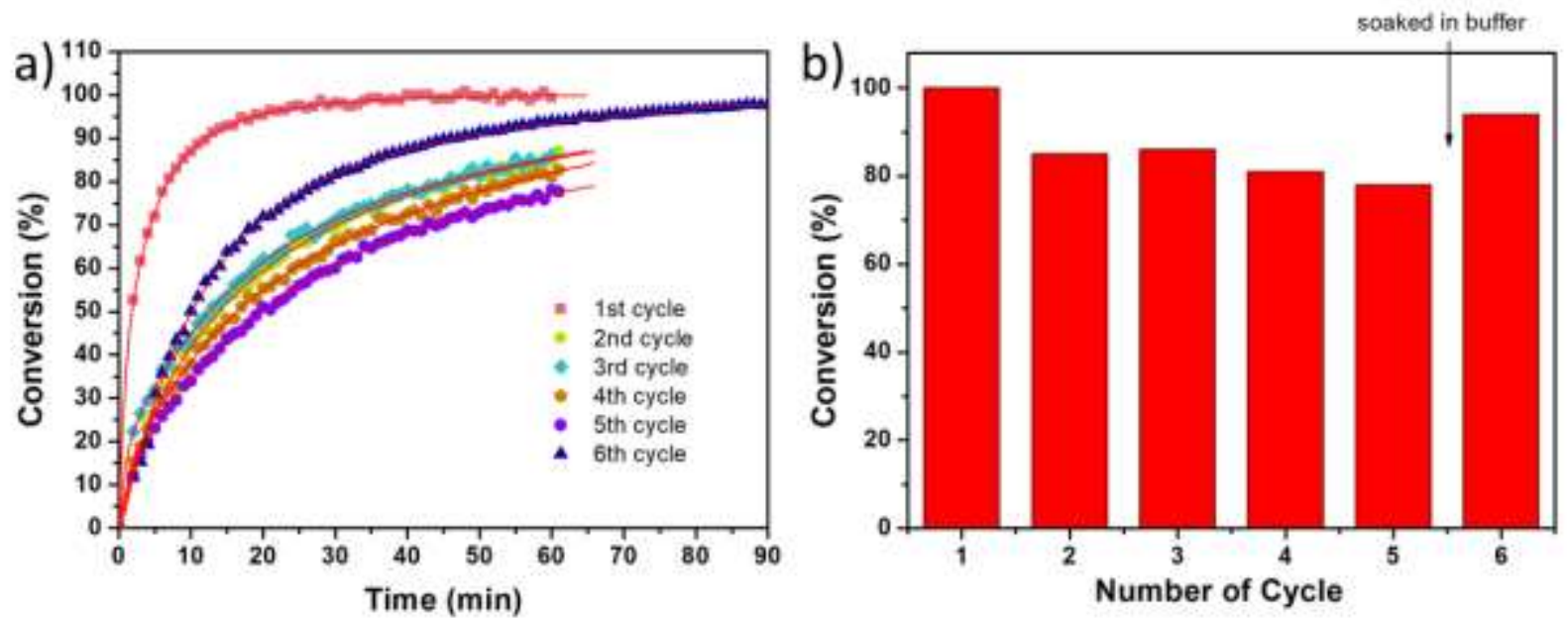

Figure S57. a) The kinetics of hydrolysis of DMNP in the presence of $1.5 \mu \mathrm{mol}$ of NU-1000-FF at room temperature for six cycles; b) The conversion of DMNP after one hour in the presence of $1.5 \mu \mathrm{mol}$ of NU1000-FF at room temperature for six cycles.

We further characterized the catalyst after 6 cycles, by XRD and SEM. It is clear from the accompanying figures that the catalyst remained crystalline and that crystallite morphology was not detectably changed.

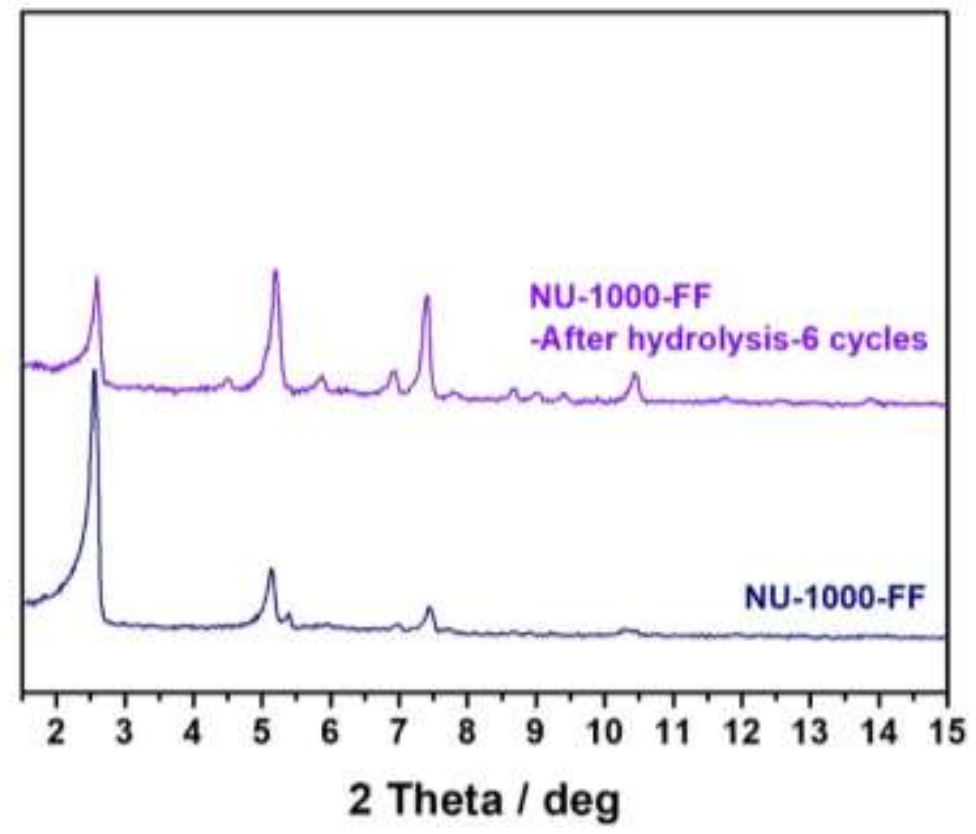

Figure S58. Comparison of PXRD patterns of NU-1000-FF and NU-1000-FF after 6 cycles of hydrolysis. 

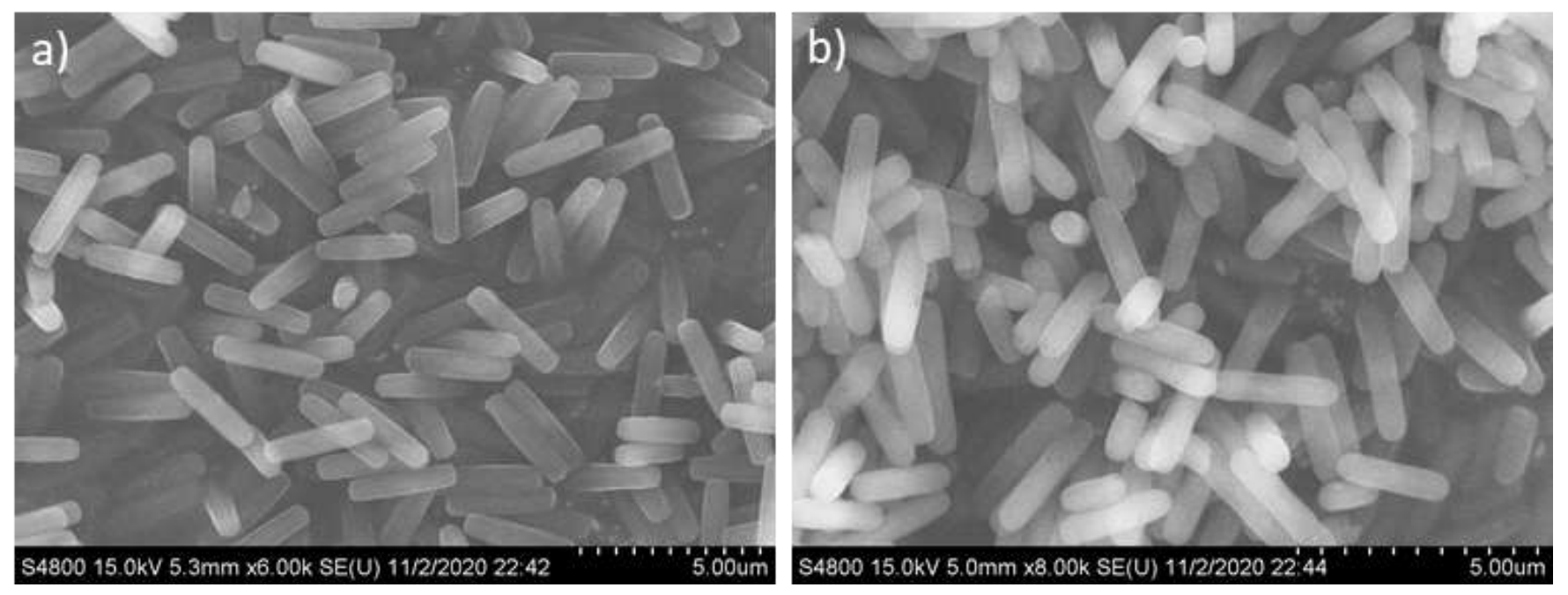

Figure S59. SEM images of NU-1000-FF before (a) and after (b) 6 cycles of hydrolysis. 